\title{
Zeros Elementares de Álgebras de Lie de Campos de Vetores
}

\author{
Luiz CARLOS MARTins JÚNior
}

Orientador: Prof. Dr. José Luis ARraut Vergara

Dissertação apresentada ao Instituto de Ciências Matemáticas de São Carlos-USP, como parte dos requisitos para obtenção do título de "Mestre em Ciências - Área: Matemática". 


\section{Agradecimentos}

A Deus, pelo dom da vida.

Ao Prof. Dr. José Luis Arraut Vergara, que além de orientador e professor, se tornou um grande amigo.

À minha noiva Myriam, que compreendeu minha ausência e me incentivou neste período com o seu carinho.

Aos meus pais Luiz Carlos e Aparecida, meus irmãos Fábio e Patricia, que mesmo à distância demonstraram seu apoio.

Aos professores Dr. Oziride Manzoli Neto e Dr $\stackrel{a}{a}$ Ires Dias, pelo apoio e atenção dispensada nas discussões de alguns fatos.

Ao $\mathrm{CNPq}$, pelo financiamento parcial deste trabalho.

Aos velhos e novos amigos, pelo apoio e companheirismo.

A todos meus professores, pelos conhecimentos transmitidos.

Aos funcionários do ICMSC em todos os escalões que, direta ou indiretamente, contribuiram para o andamento e conclusão deste trabalho. 


\section{Resumo}

Quando um grupo de Lie nilpotente age sem pontos fixos sobre uma superfície compacta $M$, a característica de Euler $\chi(M)$ de $M$ é zero [11]. Isso sugere a possibilidade de um teorema tipo Poincaré-Hopf para ações destes grupos em variedades compactas. J.F.Plante em seu artigo "Elementary Zeros of Lie Algebras of Vector Fields", [12], obtém uma caracterização dos zeros elementares dessas álgebras em dois casos: quando $\mathfrak{g}$ é nilpotente e quando $\mathfrak{g}$ é semi-simples. Ele também mostra que para uma álgebra de Lie abeliana $\mathfrak{g}$ de campos de vetores de uma superfície compacta, tal que todo zero de $\mathfrak{g}$ é elementar, existe um subconjunto $\mathcal{S} \subset \mathfrak{g}$ tal que $\mathfrak{g}-\mathcal{S}$ tem medida nula e para todo $X \in \mathcal{S}$ valem:

(a) O conjunto de zeros isolados de $X$ coincide com o conjunto (finito) de zeros de $\mathfrak{g}$;

(b) Se $p_{1}, \ldots, p_{k}$ são os zeros de g então $\sum_{i=1}^{k}$ indice $\left(X, p_{i}\right)=\chi(M)$.

Baseado neste teorema e em um exemplo, ele mostra que não é possivel definir o índice de $\mathfrak{g}$ em um zero isolado $p$ como o índice de um zero de um elemento genérico $X \in \mathcal{S}$ em $p$. Embora ele não diga, o leitor fica com a impressão que um teorema do tipo Poincaré-Hopf, como mencionado no começo, não parece existir.

Nesta dissertação faço uma exposição detalhada do artigo de J.F.Plante ilustrando com exemplos os teoremas do artigo. 


\section{Abstract}

When a nilpotent Lie group acts without fixed points on a compact surface $M$, the Euler characteristic $\chi(M)$ of $M$ vanishes [11]. This suggests the possibility of a Poincaré-Hopf type theorem for actions of these groups on compact manifolds.

J.F.Plante in his paper "Elementary Zeros of Lie Algebras of Vector Fields", [12] characterize the elementary zeros of these algebras in two cases: when $\mathfrak{g}$ is nilpotent and when $\mathfrak{g}$ is semi-simple. He also shows that for a abelian Lie algebra $\mathfrak{g}$ of vector fields of a compact surface such that every zero of $\mathfrak{g}$ is elementary, there exists a set $\mathcal{S} \subset \mathfrak{g}$ such that $\mathfrak{g}-\mathcal{S}$ has measure zero and for $X \in \mathcal{S}$ :

(a) The set of isolated zeros of $X$ coincides with the (finite) zero set of $\mathfrak{g}$;

(b) If $p_{1}, \ldots, p_{k}$ are the zeros of $\mathfrak{g}$ then $\sum_{i=1}^{k}$ index $\left(X, p_{i}\right)=\chi(M)$.

Based on this last theorem and one example, he shows that it is not possible to define the index of $\mathfrak{g}$ at an isolated zero $p$ as the index of a generic element $X \in \mathcal{S}$ at $p$. Although he does not say it, the readers get the impression that a Poincaré-Hopf type theorem, as mentioned at the beginning, does not exist.

In this dissertation I detail Plante' paper illustrating it with several examples. 


\section{Índice}

Introdução ............................. 2

1 Preliminares 1

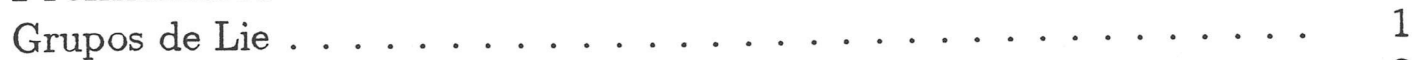

Ações de Grupos de Lie . . . . . . . . . . . . . . . . . . . 3

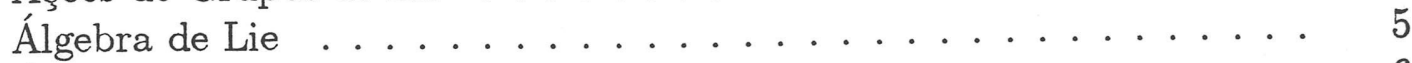

Álgebra de Lie de um Grupo de Lie . . . . . . . . . . . . . . . . . . . 6

Álgebras de Lie Solúvel, Nilpotente e Simples . . . . . . . . . . . . . . . . . 11

Cohomologia de Álgebras de Lie . . . . . . . . . . . . . . . . . . . . 14

Exemplos .......................... 15

2 Zeros Elementares de Álgebras de Lie de Campos de Vetores $\mathbf{2 5}$

O Anulamento de $\mathrm{H}^{1}$ para Álgebras de Lie Nilpotentes . . . . . . . . . 25

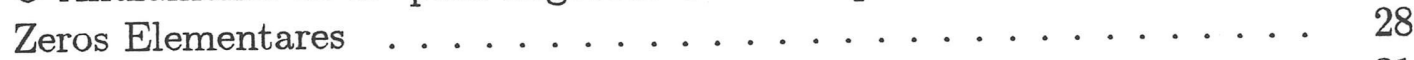

Teorema de Estabilidade . . . . . . . . . . . . . . . . . . 31

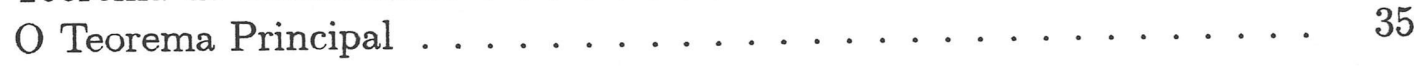

3 Apêndice $\quad 39$

Um pouco de Cohomologia. . . . . . . . . . . . . . 39

BIBLIOGRAFIA 


\section{Introdução}

Considere $X$ um campo de vetores de classe $C^{r}$ sobre uma variedade de dimensão finita $M$. Um zero de $X$ é um ponto $p \in M$ tal que $X(p)$ é o vetor nulo, e esse zero é chamado de elementar se a derivada de $X$ em $p$ é inversível. Se considerarmos o campo de vetores $X$ como uma seção em $T M$, isto é, uma aplicação $X: M \rightarrow T M$ tal que $\pi X=i d_{M}$ onde $\pi$ é a projeção natural de $T M$ em $M$, então um ponto $p \in M$ é um zero elementar se $X$ é transversal à seção nula em $p$. Desse ponto de vista vemos que zeros elementares são estáveis, isto é, persistem por pequenas perturbações de $X$. Além disso, zeros elementares são isolados.

Um resultado clássico de Poincaré [14] estabelece que "um campo de vetores sobre uma superfície compacta com característica de Euler não nula deve ter um zero em algum ponto". Esse resultado foi generelizado por Hopf [7] no seguinte sentido: Seja $M$ uma variedade fechada e $X$ um campo de vetores sobre $M$ com todos os zeros isolados. Então:

Teorema (Poincaré-Hopf): Se $\left\{p_{1}, \ldots, p_{k}\right\}$ é o conjunto de zeros de $X$ então

$$
\chi(M)=\sum_{i=1}^{k} \text { indice }\left(p_{i}\right) \text {. }
$$

Este teorema é válido em particular para campos de vetores com todos os zeros elemetares. Em 1964 Elon L. Lima [9] generalizou o resultado de Poincaré para uma família finita de campos de vetores que comutam: "Sejam $X_{1}, \ldots, X_{n}$ campos de vetores que comutam dois a dois, de classe $C^{1}$, sobre uma superfície compacta, com característica de Euler não nula. Então existe um ponto $p \in M$ tal que $X_{1}=\cdots=X_{n}=0 . "$

Seja $X$ um campo de vetores de classe $C^{1}$ sobre uma variedade fechada $M$ e $\zeta: \mathbb{R} \times M \rightarrow M$ o fluxo correspondente. Dado $p \in M$, então $X(p)=0 \Leftrightarrow \zeta(t, p)=$ $p, \forall t \in \mathbb{R}$, isto é, $p$ é um ponto fixo do fluxo $\zeta$. Seja $Y$ outro campo de vetores $C^{1}$ tal que $[X, Y]=0$, e $\eta$ o correspondente fluxo. A condição sobre o colchete de Lie implica que $\zeta(t, \eta(s, p))=\eta(s, \zeta(t, p))$. Os campos $X$ e $Y$ geram uma ação $\varphi$ do grupo de Lie $\mathbb{R}^{2}$ sobre $M$ dada por $\varphi(r, p)=\zeta(t, \eta(s, p))=\eta(s, \zeta(t, p))$ onde $p \in M$ e $r=(t, \varepsilon) \in \mathbb{R}^{2} . p \in M$ é dito um ponto fixo de $\varphi$ se e só se $p$ é um zero comum para $X$ e $Y$, isto é, $X(p)=Y(p)=0$. Analogamente qualquer número finito de campos de vetores que comutam $X_{1}, \ldots, X_{n}$ sobre uma variedade 
compacta $M$ gera uma ação $\varphi: \mathbb{R}^{n} \times M \rightarrow M$. O resultado de Elon L. Lima pode então ser parafraseado da seguinte maneira: "Toda ação do grupo de Lie $\mathbb{R}^{n}$ sobre uma superfície compacta $M$, com $\chi(M) \neq 0$, tem um ponto fixo."

É natural considerar uma situação mais geral de grupos de Lie agindo sobre M. Em [11] J.F.Plante mostrou que

"Toda ação de um grupo de Lie conexo NILPOTENTE sobre uma superfície compacta com característica de Euler não nula tem um ponto fixo."

No entanto, o resultado de Poincaré não pode mais ser generelizado para um grupo de Lie qualquer pois Plante mostra no mesmo artigo [11] que o grupo de Lie solúvel não abeliano de dimensão 2 age sem pontos fixos sobre TODA superfície compacta. Por outro lado podemos pensar na possibilidade de um teorema do índice no espírito de Poincaré-Hopf para ações por grupos de Lie nilpotentes sobre variedades compactas.

Uma ação de um grupo de Lie $G$ sobre $M$ é determinada pela ação infinitesimal sobre $M$ da álgebra de Lie $\mathfrak{g}$ de $G$ [13]. Em muitos casos é mais fácil escrever a ação infinitesimal, isto é, um homomorfismo de álgebras de Lie $A: \mathfrak{g} \rightarrow \Im(M)$ onde $\Im(M)$ é a álgebra de Lie dos campos de vetores diferenciáveis sobre $M$.

A existência de um teorema tipo Poincaré-Hopf depende da possibilidade de definir um índice para um zero de uma álgebra de Lie de campos de vetores.

Considere $\mathfrak{g}$ uma álgebra de Lie de dimensão finita de campos de vetores de classe $C^{r}$ sobre uma variedade de dimensão finita $M$. Um ponto $p \in M$ é dito ser um zero para $\mathfrak{g}$ se $X(p)=0$ para todo $X$ em $\mathfrak{g}$. Plante, no artigo [12], generaliza o conceito de zero elementar e dá uma caracterização deste para o caso em que $\mathfrak{g}$ é nilpotente. Demonstra que, em particular se $\mathfrak{g}$ é solúvel, todo zero elementar é isolado, e obtém um teorema de estabilidade para zeros elementares. Depois, mostra que para uma álgebra de Lie abeliana de campos de vetores $\mathfrak{g}$ de uma superfície compacta, cujos zeros são todos elementares, existe um subconjunto $\mathcal{S} \subset \mathfrak{g}$ tal que $\mathfrak{g}-\mathcal{S}$ tem medida nula e para todo $X \in \mathcal{S}$ valem:

(a) O conjunto de zeros isolados de $X$ coincide com o conjunto (finito) de zeros de $\mathfrak{g}$;

(b) Se $p_{1}, \ldots, p_{k}$ são os zeros de $\mathfrak{g}$ então $\sum_{i=1}^{k}$ indice $X\left(p_{i}\right)=\chi(M)$.

Baseado neste teorema e em um exemplo, ele mostra que não é possivel definir o índice de $\mathfrak{g}$ em um zero isolado $p$ como o índice de um zero de um elemento genérico $X \in \mathcal{S}$ em $p$. Embora ele não diga, o leitor fica com a impressão que um teorema do tipo Poincaré-Hopf, como mencionado no começo, não parece existir. 


\section{Capítulo 1}

\section{Preliminares}

Neste capítulo lembramos as definições básicas da teoria de Grupos de Lie, Álgebras de Lie e Cohomologia de Álgebras de Lie usadas no texto e estabelecemos a notação. A medida que avançamos no texto, alguns exemplos serão construidos. Exemplos estes que serão usados no próximo capítulo.

\section{Grupos de Lie}

Um GRUPO DE LIE G é uma variedade analítica com estrutura de grupo (com relação à uma operação *) tal que a aplicação $(x, y) \mapsto x * y^{-1}$ de $G \times G$ em $G$ é $\mathrm{C}^{\infty}$. A operação em $G$ será chamada de produto e se $x, y \in G$, então usaremos $x y$ no lugar de $x * y$.

O mais simples exemplo de grupo de Lie é o grupo aditivo $\mathbb{R}^{n}$ com sua estrutura padrão de variedade. Outro exemplo é o grupo $G L(n, \mathbb{R})$ das matrizes não singulares $n \times n$.

Um subgrupo $H \subset G$ é chamado SUBGRUPo DE LIE de $G$ se:

1. $H$ é um grupo de Lie;

2. a injeção $f: H \rightarrow G$ tal que $f(x)=x$, é uma imersão analítica;

Um critério muito útil para verificar se um subgrupo $H$, de um grupo de Lie $G$, é um subgrupo de Lie é o seguinte :

Seja $G$ um grupo de Lie e $H$ um subgrupo (abstrato) de $G$ que é um subconjunto fechado do espaço topológico $G$. Então existe uma única estrutura analítica sobre $H$ tal que $H$ é um subgrupo de Lie de $G$.

A demonstração pode ser encontrada em [16]. Este resultado diz por exemplo que $S^{1}$, visto como subgrupo de $\mathbb{C}$, é um grupo de Lie. Analogamente $S^{3}$, visto como subgrupo dos quatérnios $\mathbb{H}$, também é grupo de Lie. O subgrupo de $G L(n, \mathbb{R})$

$$
G_{A}=\left\{\left[\begin{array}{llll}
1 & 0 & 0 & \alpha \\
0 & 1 & 0 & \beta \\
0 & 0 & 1 & \gamma \\
0 & 0 & 0 & 1
\end{array}\right]: \alpha, \beta, \gamma \in \mathbb{R}\right\}
$$


também é um grupo de Lie. Também são grupos de Lie os subgrupos de $G L(n, \mathbb{R})$

$$
\begin{aligned}
& G_{N}=\left\{\left[\begin{array}{lll}
1 & \alpha & \gamma \\
0 & 1 & \beta \\
0 & 0 & 1
\end{array}\right]: \alpha, \beta, \gamma \in \mathbb{R}\right\} \\
& G_{S}=\left\{\left[\begin{array}{cccc}
e^{\gamma} & 0 & 0 & \alpha \\
0 & e^{-\gamma} & 0 & \beta \\
0 & 0 & 1 & \gamma \\
0 & 0 & 0 & 1
\end{array}\right]: \alpha, \beta, \gamma \in \mathbb{R}\right\}
\end{aligned}
$$

Seja $G$ um grupo abstrato com elemento neutro $e$. Se $K$ e $H$ são dois subgrupos de $G$, definimos $[H, K]=\left\langle h k h^{-1} k^{-1}: h \in H, k \in K\right\rangle$. Definimos, por indução, $G^{0}=G^{(0)}=G, G^{i+1}=\left[G^{i}, G\right]$ e $G^{(i+1)}=\left[G^{(i)}, G^{(i)}\right]$. Um grupo $G$ é chamado Grupo Nilpotente se existe um inteiro $n$ tal que $G^{n}=\{e\}$ e é chamado de Grupo Solúvel se existe um inteiro $n$ tal que $G^{(n)}=\{e\}$.

Um grupo de Lie $G$ é chamado Grupo de Lie Abeliano se $G$ é abeliano como grupo abstrato. Analogamente, um grupo de Lie G é um GRUPO DE LIE NilPotente (SolúVEL) se ele é nilpotente (solúvel) como um grupo abstrato. Não faz mal lembrar que um grupo abeliano é nilpotente e que um grupo nilpotente é solúvel.

É facil ver que $G_{A}$ é um grupo de Lie abeliano. Desde que

$$
\begin{gathered}
{\left[\begin{array}{lll}
1 & \alpha & \gamma \\
0 & 1 & \beta \\
0 & 0 & 1
\end{array}\right]\left[\begin{array}{lll}
1 & x & z \\
0 & 1 & y \\
0 & 0 & 1
\end{array}\right]\left[\begin{array}{ccc}
1 & -\alpha & \alpha \beta-\gamma \\
0 & 1 & -\beta \\
0 & 0 & 1
\end{array}\right]\left[\begin{array}{ccc}
1 & -x & x y-z \\
0 & 1 & -y \\
0 & 0 & 1
\end{array}\right]=} \\
=\left[\begin{array}{ccc}
1 & 0 & -\beta x+\alpha y \\
0 & 1 & 0 \\
0 & 0 & 1
\end{array}\right] \\
{\left[\begin{array}{lll}
1 & 0 & t \\
0 & 1 & 0 \\
0 & 0 & 1
\end{array}\right]\left[\begin{array}{lll}
1 & \alpha & \gamma \\
0 & 1 & \beta \\
0 & 0 & 1
\end{array}\right]\left[\begin{array}{ccc}
1 & 0 & -t \\
0 & 1 & 0 \\
0 & 0 & 1
\end{array}\right]\left[\begin{array}{ccc}
1 & -\alpha & \alpha \beta-\gamma \\
0 & 1 & -\beta \\
0 & 0 & 1
\end{array}\right]=} \\
=\left[\begin{array}{lll}
1 & 0 & 0 \\
0 & 1 & 0 \\
0 & 0 & 1
\end{array}\right]
\end{gathered}
$$

vemos que $\left[G_{N}^{1}, G_{N}\right]=\{I d\}$. Portanto $G_{N}$ é um grupo de Lie nilpotente. Analoga- 
mente, $G_{S}$ é um grupo de Lie solúvel pois

$$
\begin{gathered}
{\left[\begin{array}{cccc}
e^{\gamma} & 0 & 0 & \alpha \\
0 & e^{-\gamma} & 0 & \beta \\
0 & 0 & 1 & \gamma \\
0 & 0 & 0 & 1
\end{array}\right]\left[\begin{array}{cccc}
e^{z} & 0 & 0 & x \\
0 & e^{-z} & 0 & y \\
0 & 0 & 1 & z \\
0 & 0 & 0 & 1
\end{array}\right]\left[\begin{array}{cccc}
\frac{1}{e^{\gamma}} & 0 & 0 & -\frac{\alpha}{e^{\gamma}} \\
0 & \frac{1}{e^{-\gamma}} & 0 & -\frac{\beta}{e^{-\gamma}} \\
0 & 0 & 1 & -\gamma \\
0 & 0 & 0 & 1
\end{array}\right]\left[\begin{array}{cccc}
\frac{1}{e^{z}} & 0 & 0 & -\frac{x}{e^{z}} \\
0 & \frac{1}{e^{-z}} & 0 & -\frac{y}{e^{-z}} \\
0 & 0 & 1 & -z \\
0 & 0 & 0 & 1
\end{array}\right]} \\
=\left[\begin{array}{cccc}
1 & 0 & 0 & -x-e^{z} \alpha+e^{\gamma} x+\alpha \\
0 & 1 & 0 & -y-e^{-z} \beta+e^{-\gamma} y+\beta \\
0 & 0 & 1 \\
0 & 0 & 0 & 0 \\
0 & & 1
\end{array}\right] \\
{\left[\begin{array}{llll}
1 & 0 & 0 & \alpha \\
0 & 1 & 0 & \beta \\
0 & 0 & 1 & 0 \\
0 & 0 & 0 & 1
\end{array}\right]\left[\begin{array}{llll}
1 & 0 & 0 & x \\
0 & 1 & 0 & y \\
0 & 0 & 1 & 0 \\
0 & 0 & 0 & 1
\end{array}\right]\left[\begin{array}{cccc}
1 & 0 & 0 & -\alpha \\
0 & 1 & 0 & -\beta \\
0 & 0 & 1 & 0 \\
0 & 0 & 0 & 1
\end{array}\right]\left[\begin{array}{cccc}
1 & 0 & 0 & -x \\
0 & 1 & 0 & -y \\
0 & 0 & 1 & 0 \\
0 & 0 & 0 & 1
\end{array}\right]=} \\
=\left[\begin{array}{llll}
1 & 0 & 0 & 0 \\
0 & 1 & 0 & 0 \\
0 & 0 & 1 & 0 \\
0 & 0 & 0 & 1
\end{array}\right]
\end{gathered}
$$

ou seja, $G^{(2)}=\{I d\}$. Note que $G_{S}$ não é nilpotente visto que

$$
\begin{gathered}
{\left[\begin{array}{llll}
1 & 0 & 0 & x \\
0 & 1 & 0 & y \\
0 & 0 & 1 & 0 \\
0 & 0 & 0 & 1
\end{array}\right]\left[\begin{array}{cccc}
e^{\gamma} & 0 & 0 & \alpha \\
0 & e^{-\gamma} & 0 & \beta \\
0 & 0 & 1 & \gamma \\
0 & 0 & 0 & 1
\end{array}\right]\left[\begin{array}{cccc}
1 & 0 & 0 & -x \\
0 & 1 & 0 & -y \\
0 & 0 & 1 & 0 \\
0 & 0 & 0 & 1
\end{array}\right]\left[\begin{array}{cccc}
\frac{1}{e^{\gamma}} & 0 & 0 & -\frac{\alpha}{e^{\gamma}} \\
0 & \frac{1}{e^{-\gamma}} & 0 & -\frac{\beta}{e^{-\gamma}} \\
0 & 0 & 1 & -\gamma \\
0 & 0 & 0 & 1
\end{array}\right]=} \\
= \\
=\left[\begin{array}{cccc}
1 & 0 & 0 & -e^{\gamma} x+x \\
0 & 1 & 0 & -e^{-\gamma} y+y \\
0 & 0 & 1 & 0 \\
0 & 0 & 0 & 1
\end{array}\right]
\end{gathered}
$$

e portanto $\left[G_{S}^{1}, G_{S}\right]=G_{S}^{1}$.

\section{Ações de Grupos de Lie}

Sejam $G$ um grupo de Lie e $M$ uma variedade $C^{\infty}$. Uma AÇÃo DE Classe $C^{r}$ DO GRUPo DE Lie $G$ SOBRE $M$ é uma aplicação $\varphi: G \times M \rightarrow M$ de classe $C^{r}$ tal que:

- $\varphi(e, x)=x, \forall x \in M$, onde $e$ é o elemento neutro de $G$; 
- $\varphi(h, \varphi(g, x))=\varphi(h g, x), \forall x \in M, \forall g, h \in G$.

Em particular se $G=\mathbb{R}$, a ação $\varphi$ é chamada de FLuxo.

A ÓRBITA de um ponto $x \in M$ (pela ação $\varphi$ ) é o subconjunto de $M \mathcal{O}_{x}=$ $\mathcal{O}_{x}(\varphi)=\{\varphi(g, x) \mid g \in G\}$. O SubGRUPo DE IsotropiA de $x \in M$, pela ação $\varphi$, é o subgrupo de $G$ definido por $G_{x}=G_{x}(\varphi)=\{g \in G \mid \varphi(g, x)=x\}$. Da definição fica evidente que $G_{x}$ é um subgrupo fechado de $G$. Consideremos em $G$ a relação de equivalência $g_{1} \sim g_{2} \Leftrightarrow g_{1}^{-1} g_{2} \in G_{x}$ e seja $G / G_{x}=\left\{g G_{x}: g \in G\right\}$ o espaço das classes laterais à esquerda. Então a aplicação $G / G_{x} \rightarrow \mathcal{O}_{x}$, definido por $g G_{x} \mapsto \varphi(g, x)$, é contínua e bijetora. Se $G_{x}=G$ então $\mathcal{O}_{x}=\{x\}$ e $x$ é chamado Ponto Estacionário ou Ponto Fixo da ação. Se todo ponto é estacionário, então dizemos que $G$ age Trivialmente sobre $M$. Por outro lado, se $\mathcal{O}_{x}=M$ para algum, e portanto para todo, $x \in M$ então a ação é dita Transitiva. A ação é chamada EFETIVA se somente a identidade fixa todo ponto de $M$. Dada uma ação arbitraria de $G$ sobre $M$, o subgrupo $H$ de $G$ que age trivialmente é um subgrupo fechado e normal e portanto induz uma ação efetiva do grupo de Lie quociente $G / H$ sobre $M$.

A aplicação $\varphi: G L(n, \mathbb{R}) \times \mathbb{R}^{n} \rightarrow \mathbb{R}^{n}:(A, p) \mapsto A p$ é um exemplo de ação do grupo de Lie $G L(n, \mathbb{R})$ sobre $\mathbb{R}^{n}$. A restrição de $\varphi$ à algum subgrupo $H$ de $G L(n, \mathbb{R})$ é uma ação de $H$ sobre $\mathbb{R}^{n}$. Um outro exemplo é dado abaixo.

Açāo do grupo de Lie $S^{3}$. Seja $\mathbb{H}=\{x+y i+z j+w k: x, y, z, w$ reais $\}$ o espaço dos quatérnios, cuja base canônica é $\{1, i, j, k\}$. Denotaremos aqui por $\mathbb{R}$ o subconjunto dos quatérnios reais e por $\mathbb{R}^{3}$ o subconjunto dos quatérnios imaginários puros $\{y i+z j+w k: y, z, w$ reais $\}$. Consideremos $S^{3} \subset \mathbb{H}$ o subconjunto dos quatérnios de módulo 1 . Definimos então a ação

$$
\begin{aligned}
\varphi: S^{3} \times \mathbb{H} & \rightarrow \mathbb{H} \\
(u, p) & \mapsto u p u^{-1} .
\end{aligned}
$$

Sejam $u=(\alpha+\beta i+\gamma j+\delta k) \in S^{3}$ e $p=(x+y i+z j+w k) \in \mathbb{H}$. O subgrupo de isotropia $S_{p}^{3}$ é o conjunto dos $u$ tal que $u p u^{-1}=p$, ou seja, é o conjunto dos $u \in S^{3}$ que comutam com $p$. Desde que

$$
\begin{aligned}
u p= & (x \alpha-y \beta-z \gamma-w \delta)+i(x \beta+y \alpha+z \delta-w \gamma)+j(x \gamma-y \delta+z \alpha+w \beta)+ \\
& k(x \delta+y \gamma-z \beta+w \alpha) \\
p u= & (x \alpha-y \beta-z \gamma-w \delta)+i(x \beta+y \alpha-z \delta+w \gamma)+j(x \gamma+y \delta+z \alpha-w \beta)+ \\
& k(x \delta-y \gamma+z \beta+w \alpha)
\end{aligned}
$$

temos que $u p=p u \Longleftrightarrow\left\{\begin{array}{l}z \delta-w \gamma=-(z \delta-w \gamma) \\ w \beta-y \delta=-(w \beta-y \delta) \\ y \gamma-z \beta=-(y \gamma-z \beta)\end{array} \Longleftrightarrow\left\{\begin{array}{l}z \delta-w \gamma=0 \\ w \beta-y \delta=0 \\ y \gamma-z \beta=0\end{array}\right.\right.$.

Portanto, os subgrupos de isotropia são: 
- Se $y=z=w=0 \Rightarrow S_{p}^{3}=S^{3} \Rightarrow$ todo ponto de $\mathbb{R}$ é ponto fixo;

- A ação $\varphi$ deixa invariante os quatérnios imaginários $\mathbb{R}^{3}$. Considere $q$ a parte imáginária de $p$, isto é, $p=x+q$. Então $\varphi(u, p)=u(x+q) u^{-1}=$ $x+u q u^{-1}=x+\varphi(u, q)$, ou seja, $\mathcal{O}_{(x+p)}=x+\mathcal{O}_{p}$. Logo, basta calcular o subgrupo de isotropia $S_{i}^{3}$.

$$
S_{i}^{3}=\{\alpha+\beta i: \alpha, \beta \text { reais }\} \text {. }
$$

Portanto $\mathcal{O}_{i}=S^{3} / S_{i}^{3} \simeq S^{2}$.

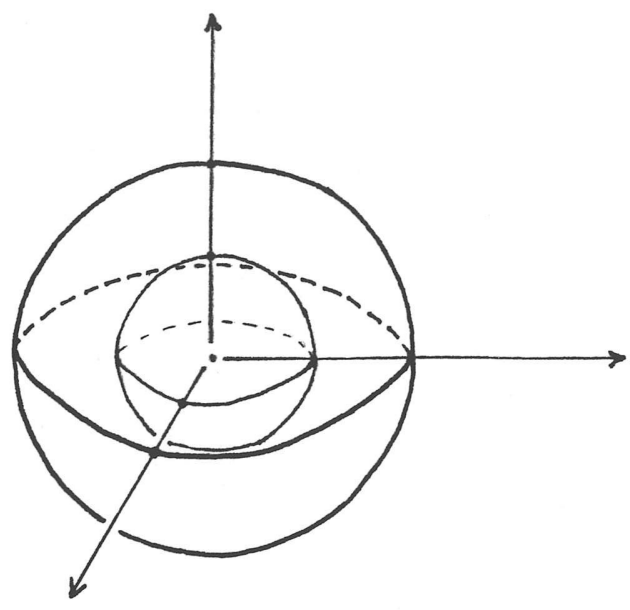

órbitas da ação no subespaço $x=0$

\section{Álgebra de Lie}

Um espaço vetorial $\mathfrak{g}$ sobre $\mathbb{R}$ é chamado uma ÁlGEBRA DE LIE se existe uma aplicação [,]: $\mathfrak{g} \times \mathfrak{g} \rightarrow \mathfrak{g}$ chamada ColChETE DE LIE (ou simplesmente colchete) tal que:

(a) $(X, Y) \mapsto[X, Y]$ é bilinear;

(b) $[X, Y]=-[Y, X], \forall X, Y \in \mathfrak{g}$ antisimétrica e;

(c) $[X,[Y, Z]]+[Y,[Z, X]]+[Z,[X, Y]]=0, \forall X, Y, Z \in \mathfrak{g} \quad$ satisfaz a identidade de Jacobi.

A condição (b) implica que $[X, X]=0, \quad \forall X \in \mathfrak{g}$.

As álgebras de Lie surgem das álgebras associativas de um modo muito simples. Seja $\mathfrak{A}$ uma álgebra associativa. Se $X, Y \in \mathfrak{A}$ então definimos o colchete de $X$ por $Y$ como

$$
[X, Y]=X Y-Y X
$$


Deste modo o conjunto $\mathfrak{g l}(V)$ dos endomorfismos de $\mathrm{V}$, onde $\mathrm{V}$ é um espaço vetorial de dimensão finita, é uma álgebra de Lie. $\mathbb{R}^{2}$ também tem estrutura de álgebra de Lie e neste caso será denotada por $\mathfrak{r}^{2}$. Outro exemplo de álgebra de Lie é o conjunto $\Im^{r}(M)$ dos campos de vetores de classe $C^{r}, r \geq 2$, em $M$. Seja $\mathbf{x}: U \subset M \rightarrow \mathbb{R}^{n}$ uma carta local. Denotemos por $\partial / \partial x_{i}$ o campo de vetores em $U$ definido por $\partial / \partial x_{i}(q)=(d \mathbf{x}(q))^{-1} \cdot e_{i}$, onde $\left\{e_{1}, \ldots, e_{n}\right\}$ é base canônica do $\mathbb{R}^{n}$. Se $X$ e $Y$ são campos de vetores em $M$, podemos escrever $X=\sum_{i=1}^{n} a_{i} \partial / \partial x_{i}$ e $Y=\sum_{i=1}^{n} b_{i} \partial / \partial x_{i}$. Então

$$
[X, Y]=\sum_{i=1}^{n} c_{i} \partial / \partial x_{i} \quad \text { onde } c_{i}=\sum_{j=1}^{n}\left(a_{j} \frac{\partial b_{i}}{\partial x_{j}}-b_{j} \frac{\partial a_{i}}{\partial x_{j}}\right) .
$$

Um subespaço vetorial $\mathfrak{h} \subset \mathfrak{g}$ é dito ser uma SUbÁlgebRA DE LIE de $\mathfrak{g}$ se para todo $X, Y \in \mathfrak{h}$ nós temos $[X, Y] \in \mathfrak{h}$.

Sejam $\mathfrak{g}$ e $\mathfrak{g}^{\prime}$ duas álgebras de Lie sobre $\mathbb{R}$. Um HoMOMORFISMO ENTRE ÁlGEBRAS DE LIE é uma aplicação linear que preserva a operação colchete, isto é, seja $\rho(X \mapsto \rho(X))$ uma aplicação linear de $\mathfrak{g}$ em $\mathfrak{g}^{\prime}$, então $\rho$ é um homomorfismo entre $\mathfrak{g}$ e $\mathfrak{g}^{\prime}$ se

$$
\rho([X, Y])=[\rho(X), \rho(Y)]
$$

Tomemos agora $\mathrm{V}$ um espaço vetorial sobre $\mathbb{R}$, não necessariamente de dimensão finita. Por uma REPREsentAÇÃo DE $\mathfrak{g}$ EM $V$ entendemos uma aplicação linear

$$
\rho: X \mapsto \rho(X), \quad(X \in \mathfrak{g})
$$

de $\mathfrak{g}$ em $\mathfrak{g l}(V)$ tal que $\rho([X, Y])=\rho(X) \rho(Y)-\rho(Y) \rho(X), \quad \forall X, Y \in \mathfrak{g}$.

Dado uma álgebra de Lie $\mathfrak{g}$, para qualquer $X \in \mathfrak{g}$ a aplicação

$$
\begin{aligned}
\operatorname{adX}: \mathfrak{g} & \rightarrow \mathfrak{g} \\
Y & \mapsto[X, Y]
\end{aligned}
$$

é um endomorfismo de $\mathfrak{g}$. Por cálculo direto vemos que a aplicação $a d: X \mapsto a d X$ é uma representação de $\mathfrak{g}$ chamada REPRESENTAÇÃo ADJUNTA de $\mathfrak{g}$. O kernel de $a d$ é o conjunto de todos os $X \in \mathfrak{g}$ tais que $[X, Y]=0$ para todo $Y \in \mathfrak{g}$; ele é chamado CENTRO de $\mathfrak{g}$.

Se $\mathrm{V}$ tem dimensão finita, então dizer que $\rho$ é uma representação de $\mathfrak{g} \mathrm{em} \mathrm{V}$ é equivalente a dizer que $\rho$ é um homomorfismo de $\mathfrak{g}$ em $\mathfrak{g l}(V)$.

No que segue, $\mathfrak{g}$ é uma álgebra de Lie de dimensão finita sobre $\mathbb{R}$ e $\mathrm{V}$ é um espaço vetorial real também de dimensão finita.

\section{Álgebra de Lie de um Grupo de Lie}

Dado um elemento $a$ de um grupo de Lie $G$, a função $L_{a}: G \rightarrow G$, definida por $g \mapsto a g$, é chamada TranslaÇão À Esquerda. Como o produto em $G$ é diferenciável e a inversa de $L_{a}$ é $L_{a^{-1}}$, então $L_{a}$ é um difeomorfismo de $G$ em $G$. 
Um campo de vetores $X$ sobre $G$ é dito ser INVARIANTE À Esquerda se $X$ é invariante por todas as translações à esquerda de $G$, isto é,

$$
X \circ L_{a}(g)=D L_{a} \circ X(g), \quad \forall g, a \in G \text {. }
$$

Denotemos por $\mathfrak{g}$ o conjunto dos campos de vetores invariantes à esquerda sobre $G$. Mostra-se que $\mathfrak{g}$ tem estrutura de álgebra de Lie, ver [3]. g é chamada de Álgebra de LiE do Grupo de LIE G.

Todo campo de vetores invariantes à esqueda $X \in \mathfrak{g}$ fica determinado pelo seu valor no elemento neutro $e$ de $G$, pois

$$
X(a)=X(a e)=X \circ L_{a}(e)=D L_{a} \circ X(e) \quad \forall a \in G .
$$

Por outro lado, na demonstração do resultado abaixo mostra-se que se $v \in$ $T_{e} G$, então $g \mapsto D L_{g}(v)$ é um campo de vetores invariante cujo valor em $e$ é $v$. Um resultado útil sobre a álgebra de Lie de um grupo é o seguinte:

"A função $\mathfrak{g} \rightarrow T_{e} G$ definida por $X \mapsto X(e)$ é um isomorfismo do espaço vetorial $\mathfrak{g}$ sobre $T_{e} G$. Em particular $\operatorname{dim} G=\operatorname{dim} \mathfrak{g}$. "

A demonstração pode ser encontrada em [3]. Podemos usar esse isomorfismo para colocar a estrutura de álgebra de Lie sobre $T_{e} G$. Ás vezes chamaremos $T_{e} G$ de álgebra de Lie de $G$ e a denotaremos por $\mathfrak{g}$ quando não ouver confusão.

Álgebra de Lie de $S^{3}$. Sejam $p=a+b i+c j+d k, \quad u=x+y i+z j+$ $w k, \quad u, p \in \mathbb{H} \operatorname{com}\|u\|=\|p\|=1$ e $T_{p}(u)=\left(x \frac{\partial}{\partial x}+y \frac{\partial}{\partial y}+z \frac{\partial}{\partial z}+w \frac{\partial}{\partial w}\right)_{p} \mathrm{a}$ aplicação que troca a origem do vetor $u$ de 0 por $p$.

Suponhamos que $A$ e $B$ são espaços vetoriais de dimensão finita, $\phi: A \rightarrow B$ uma aplicação linear e $p \in A$. Então

$$
D \phi \circ T_{p}=T_{\phi(p)} \circ \phi
$$
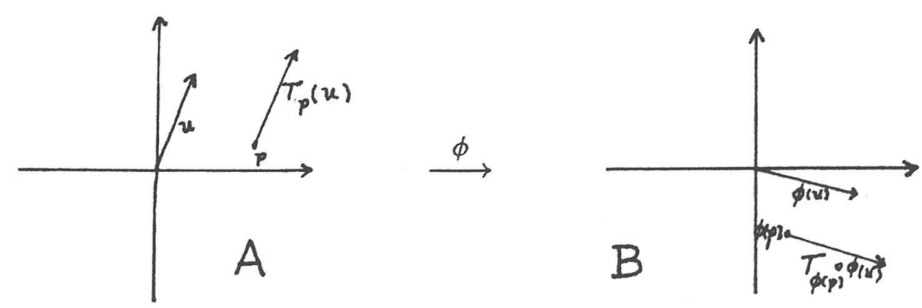

Sejam :

$$
\begin{aligned}
& \bar{I}(u)=u i=-y+x i+w j-z k \\
& \bar{J}(u)=u j=-z-w i+x j+y k \\
& \bar{K}(u)=u k=-w+z i-y j+x k
\end{aligned}
$$




$$
\begin{aligned}
& I(u)=\frac{1}{2}\left(T_{u} \circ \bar{I}\right)(u)=\frac{1}{2}\left(-y \frac{\partial}{\partial x}+x \frac{\partial}{\partial y}+w \frac{\partial}{\partial z}-z \frac{\partial}{\partial w}\right)_{u} \\
& J(u)=\frac{1}{2}\left(T_{u} \circ \bar{J}\right)(u)=\frac{1}{2}\left(-z \frac{\partial}{\partial x}-w \frac{\partial}{\partial y}+x \frac{\partial}{\partial z}+y \frac{\partial}{\partial w}\right)_{u} \\
& K(u)=\frac{1}{2}\left(T_{u} \circ \bar{K}\right)(u)=\frac{1}{2}\left(-w \frac{\partial}{\partial x}+z \frac{\partial}{\partial y}-y \frac{\partial}{\partial z}+x \frac{\partial}{\partial w}\right)_{u}
\end{aligned}
$$

Desde que $T_{u}(u) \cdot I(u)=T_{u}(u) \cdot J(u)=T_{u}(u) \cdot K(u)=0$, temos então que $\{I(u), J(u), K(u)\} \subset T_{u} S^{3}$. Logo $I, J, K$ são campos vetoriais sobre $S^{3}$.

Lembremos que $L_{p}$ é a translação a esquerda tal que $L_{p}(u)=p u$. Logo,

$$
\left(L_{p} \circ \bar{I}\right)(u)=L_{p}(u i)=p(u i)=(p u) i=\bar{I}(p u)=\left(\bar{I} \circ L_{p}\right)_{u}
$$

Logo,

$$
\begin{aligned}
D L_{p} \circ I(u) & =D L_{p} \circ\left(\frac{1}{2}\left(T_{u} \circ \bar{I}\right)\right)(u)=\frac{1}{2}\left(D L_{p} \circ T_{u}\right) \circ \bar{I}(u) \stackrel{1.1}{=} \\
& =\frac{1}{2}\left(T_{p u} \circ L_{p}\right) \circ \bar{I}(u)=\frac{1}{2} T_{p u} \circ\left(L_{p} \circ \bar{I}\right)(u) \stackrel{1.2}{=} \\
& =\frac{1}{2}\left(T_{p u} \circ \bar{I}\right) \circ L_{p}(u)=I \circ L_{p}(u)
\end{aligned}
$$

Portanto $I$ é um campo de vetores sobre $S^{3}$ invariante a esquerda. Analogamente para os campos vetoriais $J$ e $K$. Além disso, por cálculo direto, temos que $[I, J]=K,[I, K]=-J,[J, K]=I$. Desde que o espaço vetorial gerado por $\{i, j, k\}$ é isomorfo à $T_{1} S^{3}$ temos que $[i, j]=k,[i, k]=-j,[j, k]=i$. Esse espaço vetorial será denotado por $\mathfrak{s}^{3}$ e é a álgebra de Lie do grupo de Lie $S^{3}$.

Chamamos um homomorfismo diferenciável $\tau: \mathbb{R} \rightarrow G$ de SubGrupo A 1PARÂMetro de G. O seguinte resultado pode ser encontrado em [BC].

"Seja $G$ um grupo de Lie, $v \in T_{e} G, X \in \mathfrak{g}$ um campo de vetores invariante a esquerda tal que $X(e)=v$. Então existe exatamente um subgrupo a 1-parâmetro $\tau: \mathbb{R} \rightarrow G$ tal que

$$
\tau^{\prime}(0)=X(e)=v . "
$$

Com isso, outra maneira de se calcular a álgebra de Lie de um grupo de Lie é através de subgrupos a 1-parâmetro de $G$.

af: a álgebra de Lie nāo abeliana de dimensāo 2. O grupo de Lie das transformações afins da reta $A f$ é o conjunto de todos os difeomorfismos afins $f: \mathbb{R} \rightarrow \mathbb{R}$ com a operação composição

$$
\begin{aligned}
\circ: A f \times A f & \rightarrow A f \\
(f, g) & \mapsto f \circ g
\end{aligned}
$$


As transformações afins são aquelas da forma $f(x)=\alpha x+\beta$, onde $\alpha, \beta \in$ $\mathbb{R}$ com $\alpha \neq 0$, são fixos. Neste texto consideramos Af como sendo somente a componente conexa da identidade, ou seja, quando $\alpha>0$. Dadas $f, g \in A f$, $f(x)=\alpha x+\beta$ e $g(x)=\alpha^{\prime} x+\beta^{\prime}$, a composição $(f \circ g)(x)=\left(\alpha \alpha^{\prime}\right) x+\left(\alpha \beta^{\prime}+\beta\right)$ também é afim. Não é difícil ver que existe um isomorfismo entre Af e o subgrupo de $G L(2, \mathbb{R})$ definido pelo conjunto de matrizes

$$
\left[\begin{array}{ll}
\alpha & \beta \\
0 & 1
\end{array}\right] \text { tal que } \alpha, \beta \in \mathbb{R}, \alpha>0
$$

com a operação produto.

As aplicações $a: \mathbb{R} \rightarrow A f, b: \mathbb{R} \rightarrow A f$, tal que

$$
a(t)=\left[\begin{array}{cc}
e^{t} & 0 \\
0 & 1
\end{array}\right], \quad b(t)=\left[\begin{array}{ll}
1 & t \\
0 & 1
\end{array}\right]
$$

são subgrupos a 1-parâmetro de Af.

Denotemos por $X$ e $Y$ os vetores $a^{\prime}(0)$ e $b^{\prime}(0)$ :

$$
X=\left[\frac{\partial}{\partial t} a\right]_{t=0}=\left[\begin{array}{ll}
1 & 0 \\
0 & 0
\end{array}\right], \quad Y=\left[\frac{\partial}{\partial t} b\right]_{t=0}=\left[\begin{array}{ll}
0 & 1 \\
0 & 0
\end{array}\right]
$$

e por af a álgebra de Lie de $A f$. Desde que $X$ e $Y$ são l.i., então $\{X, Y\}$ é base para af. Note que $[X, Y]=Y$.

$\mathfrak{g}_{A}:$ a álgebra de Lie do grupo de Lie $\mathbf{G}_{A}$. As aplicações $a: \mathbb{R} \rightarrow G_{A}$, $b: \mathbb{R} \rightarrow G_{A}, c: \mathbb{R} \rightarrow G_{A}$, tal que

$$
a(t)=\left[\begin{array}{llll}
1 & 0 & 0 & t \\
0 & 1 & 0 & 0 \\
0 & 0 & 1 & 0 \\
0 & 0 & 0 & 1
\end{array}\right], \quad b(t)=\left[\begin{array}{llll}
1 & 0 & 0 & 0 \\
0 & 1 & 0 & t \\
0 & 0 & 1 & 0 \\
0 & 0 & 0 & 1
\end{array}\right], \quad c(t)=\left[\begin{array}{llll}
1 & 0 & 0 & 0 \\
0 & 1 & 0 & 0 \\
0 & 0 & 1 & t \\
0 & 0 & 0 & 1
\end{array}\right]
$$

são subgrupos a 1-parâmetro de $G_{A}$.

Denotemos por $X, Y, Z$ os vetores $a^{\prime}(0), b^{\prime}(0), c^{\prime}(0)$ :

$$
\begin{aligned}
& X=\left[\frac{\partial}{\partial t} a\right]_{t=0}=\left[\begin{array}{llll}
0 & 0 & 0 & 1 \\
0 & 0 & 0 & 0 \\
0 & 0 & 0 & 0 \\
0 & 0 & 0 & 0
\end{array}\right], Y=\left[\frac{\partial}{\partial t} b\right]_{t=0}=\left[\begin{array}{llll}
0 & 0 & 0 & 0 \\
0 & 0 & 0 & 1 \\
0 & 0 & 0 & 0 \\
0 & 0 & 0 & 0
\end{array}\right], \\
& Z=\left[\frac{\partial}{\partial t} c\right]_{t=0}=\left[\begin{array}{llll}
0 & 0 & 0 & 0 \\
0 & 0 & 0 & 0 \\
0 & 0 & 0 & 1 \\
0 & 0 & 0 & 0
\end{array}\right]
\end{aligned}
$$


e por $\mathfrak{g}_{A}$ a álgebra de Lie de $G_{A}$ gerada pelos vetores $X, Y$ e $Z$. Note que $[X, Y]=$ $[X, Z]=[Y, Z]=0$.

$\mathfrak{g}_{N}:$ a álgebra de Lie do grupo de Lie $\mathbf{G}_{N}$. As aplicações $a: \mathbb{R} \rightarrow G_{N}$, $b: \mathbb{R} \rightarrow G_{N}, c: \mathbb{R} \rightarrow G_{N}$, tal que

$$
a(t)=\left[\begin{array}{lll}
1 & t & 0 \\
0 & 1 & 0 \\
0 & 0 & 1
\end{array}\right], \quad b(t)=\left[\begin{array}{lll}
1 & 0 & 0 \\
0 & 1 & t \\
0 & 0 & 1
\end{array}\right], \quad c(t)=\left[\begin{array}{lll}
1 & 0 & t \\
0 & 1 & 0 \\
0 & 0 & 1
\end{array}\right]
$$

são subgrupos a 1-parâmetro de $G_{N}$.

Denotemos por $X, Y, Z$ os vetores $a^{\prime}(0), b^{\prime}(0), c^{\prime}(0)$ :

$$
\begin{aligned}
& X=\left[\frac{\partial}{\partial t} a\right]_{t=0}=\left[\begin{array}{lll}
0 & 1 & 0 \\
0 & 0 & 0 \\
0 & 0 & 0
\end{array}\right], Y=\left[\frac{\partial}{\partial t} b\right]_{t=0}=\left[\begin{array}{lll}
0 & 0 & 0 \\
0 & 0 & 1 \\
0 & 0 & 0
\end{array}\right], \\
& Z=\left[\frac{\partial}{\partial t} c\right]_{t=0}=\left[\begin{array}{lll}
0 & 0 & 1 \\
0 & 0 & 0 \\
0 & 0 & 0
\end{array}\right],
\end{aligned}
$$

e por $\mathfrak{g}_{N}$ a álgebra de Lie do grupo de Lie $G_{N}$ onde $\{X, Y, Z\}$ é base para $\mathfrak{g}_{N}$. Note que $[X, Y]=Z,[X, Z]=[Y, Z]=0$.

$\mathfrak{g}_{S}:$ a álgebra de Lie do grupo de Lie $\mathbf{G}_{S}$. As aplicações $a: \mathbb{R} \rightarrow G_{S}$, $b: \mathbb{R} \rightarrow G_{S}, c: \mathbb{R} \rightarrow G_{S}$, tal que

$$
a(t)=\left[\begin{array}{llll}
1 & 0 & 0 & t \\
0 & 1 & 0 & 0 \\
0 & 0 & 1 & 0 \\
0 & 0 & 0 & 1
\end{array}\right], \quad b(t)=\left[\begin{array}{llll}
1 & 0 & 0 & 0 \\
0 & 1 & 0 & t \\
0 & 0 & 1 & 0 \\
0 & 0 & 0 & 1
\end{array}\right], \quad c(t)=\left[\begin{array}{cccc}
e^{t} & 0 & 0 & 0 \\
0 & e^{-t} & 0 & 0 \\
0 & 0 & 1 & t \\
0 & 0 & 0 & 1
\end{array}\right]
$$

são subgrupos a 1-parâmetro de $G_{S}$.

Denotemos por $X, Y, Z$ os vetores $a^{\prime}(0), b^{\prime}(0), c^{\prime}(0)$ :

$$
\begin{aligned}
& X=\left[\frac{\partial}{\partial t} a\right]_{t=0}=\left[\begin{array}{llll}
0 & 0 & 0 & 1 \\
0 & 0 & 0 & 0 \\
0 & 0 & 0 & 0 \\
0 & 0 & 0 & 0
\end{array}\right], \quad Y=\left[\frac{\partial}{\partial t} b\right]_{t=0}=\left[\begin{array}{llll}
0 & 0 & 0 & 0 \\
0 & 0 & 0 & 1 \\
0 & 0 & 0 & 0 \\
0 & 0 & 0 & 0
\end{array}\right], \\
& Z=\left[\frac{\partial}{\partial t} c\right]_{t=0}=\left[\begin{array}{cccc}
1 & 0 & 0 & 0 \\
0 & -1 & 0 & 0 \\
0 & 0 & 0 & 1 \\
0 & 0 & 0 & 0
\end{array}\right]
\end{aligned}
$$

Denotemos por $\mathfrak{g}_{S}$ a álgebra de Lie do grupo de Lie $G_{S}$ onde $\{X, Y, Z\}$ é base para $\mathfrak{g}_{S}$. Note que $[X, Y]=0,[X, Z]=-X,[Y, Z]=Y$. 


\section{Álgebras de Lie Solúvel, Nilpotente e Simples}

Sejam $\mathfrak{h}$ e $\mathfrak{s}$ subespaços lineares de $\mathfrak{g}$. Denotemos por $[\mathfrak{h}, \mathfrak{s}]$ o subespaço de $\mathfrak{g}$ gerado por todos os produtos $[X, Y]$ para $X \in \mathfrak{h}$ e $Y \in \mathfrak{s}$. Um subespaço $\mathfrak{h}$ de $\mathfrak{g}$ é chamado SubÁlgebra se $[\mathfrak{h}, \mathfrak{h}] \subseteq \mathfrak{h}$; e é chamado de IDEAL se $[\mathfrak{g}, \mathfrak{h}] \subseteq \mathfrak{h}$. Em particular $[\mathfrak{g}, \mathfrak{g}]$ é um ideal de $\mathfrak{g}$. A notação usual para dizer que $\mathfrak{h}$ é ideal de $\mathfrak{g}$ é $\mathfrak{h} \triangleleft \mathfrak{g}$.

Se $\pi: \mathfrak{g} \rightarrow \mathfrak{g}^{\prime}$ é um homomorfismo, então $\pi(\mathfrak{g})$ é uma subálgebra de $\mathfrak{g}^{\prime}$ e o kernel de $\pi$ é um ideal de $\mathfrak{g}$. Por outro lado, se $\mathfrak{g}$ é uma álgebra de Lie e $\mathfrak{h}$ um ideal de $\mathfrak{g}$, denotemos por $\mathfrak{g}^{\prime}$ o espaço vetorial quociente $\mathfrak{g} / \mathfrak{h}$, e $\pi$ a aplicação linear canônica de $\mathfrak{g}$ sobre $\mathfrak{g}^{\prime}$. Para $\bar{X}=\pi(X)$ e $\bar{Y}=\pi(Y)$ seja

$$
[\bar{X}, \bar{Y}]=\pi([X, Y]) \text {. }
$$

Não é difícil mostar que $[\bar{X}, \bar{Y}]$ está bem definida e satisfaz a definição de colchete. Logo $\mathfrak{g}^{\prime}$ é uma álgebra de Lie e $\pi$ é portanto um homomorfismo de $\mathfrak{g}$ sobre $\mathfrak{g}^{\prime}$ com kernel $\mathfrak{h}$. Chamamos a álgebra $\mathfrak{g} / \mathfrak{h}$ de Quociente de $\mathfrak{g}$ por $\mathfrak{h}$. O resultado abaixo pode ser encontrado em [16].

"Sejam $G$ um grupo de Lie com álgebra de Lie $\mathfrak{g}$ e $H$ um subgrupo de Lie fechado normal com álgebra de Lie h. Então $G / H$ é um grupo de Lie e sua álgebra de Lie é isomorfa a álgebra quociente $\mathfrak{g} / \mathfrak{h}$. "

Denotemos por $\mathfrak{g}^{(1)}$ o ideal $[\mathfrak{g}, \mathfrak{g}]$ e defina por indução

$$
\mathfrak{g}^{(k+1)}=\left[\mathfrak{g}^{(k)}, \mathfrak{g}^{(k)}\right] \text {. }
$$

Da Identidade de Jacobi temos a sequência $\mathfrak{g} \triangleright \mathfrak{g}^{(1)} \triangleright \mathfrak{g}^{(2)} \triangleright \ldots$. Se existe $n$ inteiro tal $\mathfrak{g}^{(n)}=\{0\}$, então dizemos que $\mathfrak{g}$ é uma álgebra de Lie SoLúveL.

Proposiçāo. Seja $G$ um grupo de Lie conexo e g sua álgebra de Lie. Então, $G$ é solúvel se e só se g é solúvel.

Portanto $\mathfrak{g}_{S}$ é uma álgebra de Lie solúvel. A prova pode ser vista em [16].

Cabe aqui observar que se $\mathfrak{g}$ é solúvel, então subálgebras e quocientes também serão. Mais ainda, se $\mathfrak{g} / \mathfrak{h}$ e $\mathfrak{h}$ são solúveis então $\mathfrak{g}$ também é solúvel. Logo, se $\mathfrak{h}$ e $\mathfrak{s}$ são ideais solúveis de $\mathfrak{g}, \mathfrak{h} / \mathfrak{h} \cap \mathfrak{s}$ também é solúvel. Desde que $(\mathfrak{h}+\mathfrak{s}) / \mathfrak{s} \cong \mathfrak{h} / \mathfrak{h} \cap \mathfrak{s}$ temos que $(\mathfrak{h}+\mathfrak{s})$ também é solúvel. Como $\mathfrak{g}$ tem dimensão finita, existe um único ideal maximal solúvel de g: a soma dos ideais solúveis de $\mathfrak{g}$. Esse ideal maximal solúvel é chamado de RADICAL de $\mathfrak{g}$ e é denotado por radg.

Chamamos uma álgebra de Lie g SEMI-SIMPLES se radg $=\{0\}$. Não é difícil ver que se $\mathfrak{g}$ é semi-simples então $[\mathfrak{g}, \mathfrak{g}]=\mathfrak{g}$. Dizemos que um grupo de Lie $G$ é semi-simples se sua álgebra de Lie é semi-simples. 
Se $\mathfrak{g}$ não admite ideais próprios então $\mathfrak{g}$ é semi-simples ou tem dimensão 1 .

Uma álgebra de Lie $\mathfrak{g}$ é dita ser SIMPLES se $\mathfrak{g}$ é não abeliano e não possui ideais próprios, isto é, $\mathfrak{g}$ é simples se e só se $\mathfrak{g}$ é semi-simples e não tem ideais próprios. Por exemplo $\mathfrak{s}^{3}$ é semi-simples. Definimos um grupo de Lie $G$ como simples se sua álgebra de Lie é simples. Portanto $S^{3}$ é um grupo de Lie simples.

Denotemos por $\mathfrak{g}^{1}$ o ideal $[\mathfrak{g}, \mathfrak{g}]$ e defina por indução

$$
\mathfrak{g}^{k+1}=\left[\mathfrak{g}, \mathfrak{g}^{k}\right]
$$

Novamente a Identidade de Jacobi nos fornece a sequência $\mathfrak{g} \triangleright \mathfrak{g}^{1} \triangleright \mathfrak{g}^{2} \triangleright \cdots$. Se existe $n$ inteiro tal que $\mathfrak{g}^{n}=\{0\}$, então dizemos que $\mathfrak{g}$ é uma álgebra de Lie Nilpotente. Outra maneira equivalente de ver se uma álgebra de Lie é nilpotente é mostrar que $a d X$ é um endomorfismo nilpotente para todo $X \in \mathfrak{g}$. Da definição vemos que uma álgebra de Lie nilpotente é solúvel. Cabe observar aqui que se $\mathfrak{g}$ é nilpotente, então subálgebras e quocientes também são nilpotentes.

Proposiçāo. Seja $G$ um grupo de Lie real conexo e com álgebra de Lie g. Então $G$ é um grupo de Lie nilpotente se e só se g é uma álgebra de Lie nilpotente. em [16].

Com isso, $\mathfrak{g}_{N}$ é uma álgebra de Lie nilpotente. A demonstração pode ser vista

Em [8, pg.11] encontramos uma descrição das álgebras de Lie de dimensão 2 e 3. Se $\{X, Y\}$ é base para uma álgebra de Lie de dimensão 2 , então $\mathfrak{g}$ é isomorfo a uma das seguintes álgebras:

- ABELIANA. $[X, Y]=0$; como por exemplo $\mathfrak{r}^{2}$.

- NÃO ABELIANA. $[X, Y]=Y$; como por exemplo af.

Se $\{X, Y, Z\}$ é base $\mathrm{p} /$ uma álgebra de Lie $\mathfrak{g}$, então $\mathfrak{g}$ é isomorfo a uma das seguintes álgebras (ver também [1]):

- ABELIANA. $[X, Y]=[X, Z]=[Y, Z]=0$; como por exempo $\mathfrak{g}_{A}$.

- NILPOTENTE. $[X, Y]=Z,[X, Z]=[Y, Z]=0$; como por exemplo $\mathfrak{g}_{N}$.

- SOLÚVEL. $[X, Y]=0,[X, Z]=\alpha X+\beta Y,[Y, Z]=\gamma X+\delta Y$ onde

$$
A=\left[\begin{array}{cc}
\alpha & \beta \\
\gamma & \delta
\end{array}\right] \quad \text { é não singular; }
$$

como por exemplo $\mathfrak{g}_{S}$, e neste caso $\alpha=-1, \delta=1, \beta=\gamma=0$.

- SIMPLES. 


$$
\begin{aligned}
& -[X, Y]=Z,[X, Z]=-Y,[Y, Z]=X ; \text { como por exemplo } \mathfrak{s}^{3} \\
& -[X, Y]=-2 Y,[X, Z]=2 Z,[Y, Z]=X .
\end{aligned}
$$

Uma representação $\rho$ de uma álgebra de Lie $\mathfrak{g}$ em um espaço vetorial $V$ é chamada de NIL REPRESENTAÇÃo se $\rho(X)$ é nilpotente para todo $X \in \mathfrak{g}$.

Teorema 1.1 (de Engel) Se $\rho$ é uma nil representação de $\mathfrak{g}$ em $V$, então $\exists v \in V$ não nulo tal que

$$
\rho(X) v=0, \quad \forall X \in \mathfrak{g} .
$$

Uma demonstação pode ser encontrada em [16].

Corolário 1.2 O centro de uma álgebra de Lie nilpotente $\mathfrak{g}$ tem dimensão pelo menos igual a 1.

Prova. De fato, se $\mathfrak{g}$ é nilpotente então a representação adjunta é uma nil representação de $\mathfrak{g}$. Portanto, existe $Y \in \mathfrak{g}$ tal que $\operatorname{ad} X(Y)=[X, Y]=0, \forall X \in \mathfrak{g}$.

Lema 1.3 Se $\mathfrak{g}$ é nilpotente e $\operatorname{dim} \mathfrak{g} \geq 2$ então $\operatorname{dim}(\mathfrak{g} /[\mathfrak{g}, \mathfrak{g}]) \geq 2$.

Prova. O lema é verdadeiro para dim $\mathfrak{g}=2$ pois a única álgebra de Lie nilpotente é a abeliana. Assuma que o lema é verdadeiro para dim $\mathfrak{g}=n-1(n \geq$ 3). Se $\operatorname{dim} \mathfrak{g}=n$, o corolário anterior garante que existe um ideal $\mathfrak{h} \subset \mathfrak{k e r}$ ad tal que $\operatorname{dim} \mathfrak{h}=1$. Como $\mathfrak{g} / \mathfrak{h}$ tem dimensão $(n-1)$ então

$$
\operatorname{dim}\left(\frac{\mathfrak{g} / \mathfrak{h}}{[\mathfrak{g} / \mathfrak{h}, \mathfrak{g} / \mathfrak{h}]}\right) \geq 2
$$

Mas os elementos de $[\mathfrak{g} / \mathfrak{h}, \mathfrak{g} / \mathfrak{h}]$ são da forma $g \mathfrak{h} g^{\prime} \mathfrak{h}-g^{\prime} \mathfrak{h} g \mathfrak{h}=g g^{\prime} \mathfrak{h}-g^{\prime} g \mathfrak{h}=$ $\left(g g^{\prime}-g^{\prime} g\right) \mathfrak{h}$. Portanto $[\mathfrak{g} / \mathfrak{h}, \mathfrak{g} / \mathfrak{h}]=[\mathfrak{g}, \mathfrak{g}] / \mathfrak{h}$, e o lema é verdadeiro.

Existe uma generalização do processo de encontrar uma forma canônica de Jordan da matriz de um endomorfismo de $V$, isto é, encontrar "formas de Jordan" simultaneamente para uma álgebra de Lie nilpotente de endomorfismos. A demonstração do próximo teorema pode ser encontrada em [8, pg.50].

Teorema 1.4 Considere $V$ um espaço vetorial complexo de dimensão finita $e \mathfrak{h}$ uma subálgebra de Lie nilpotente complexa de $\mathfrak{g l}(V)$. Então existem $h_{i}: \mathfrak{h} \rightarrow$ $\mathbb{C}, i=1, \ldots, k$, lineares tal que

$$
V=W_{1} \bigoplus \cdots \bigoplus W_{k}
$$


onde $W_{i}=\left\{u \in V\right.$ : para todo $\left.X \in \mathfrak{h},\left(X-h_{i}(X) I\right)^{m} u=0\right\}$ são h-invariantes. Além disso, existe uma base de $V$ tal que para todo $X \in \mathfrak{h}$, a restrição de $X$ a $W_{i}$, relativo a essa base, têm a forma

$$
[X]_{i}=\left[\begin{array}{ccc}
h_{i}(X) & & * \\
& \ddots & \\
0 & & h_{i}(X)
\end{array}\right] .
$$

Ou seja, a matriz do endomorfismo $X \in \mathfrak{h}$, relativo a essa base tem a forma

$$
[X]=\left[\begin{array}{ccc}
{[X]_{1}} & & 0 \\
& {[X]_{2}} & \\
0 & & \ddots
\end{array}\right] .
$$

Seja $V$ um espaço vetorial sobre $\mathbb{R}$. Uma CoMplexificAÇÃo de $V$ é um espaço vetorial $V_{c}$ obtido extendendo o corpo base $\mathbb{R}$ para o corpo base $\mathbb{C}$, isto é,

$$
V_{c}=\{u+i v: u, v \in V\}
$$

Note que $V \subset V_{c}$ pela identificação $V=V+i\{0\}$. Em [8] encontramos a seguinte proposição:

Proposiçāo 1.5 Uma álgebra de Lie g é comutativa, nipotente ou solúvel se e só se $\mathfrak{g}_{c}$ é, respectivamente, comutativa, nilpotente ou solúvel. Além disso, se $\rho: \mathfrak{g} \rightarrow$ $\mathfrak{g l}(V)$ é uma ação de $\mathfrak{g}$ em $V$, então $\rho$ tem uma única extensão $\rho_{c}: \mathfrak{g}_{c} \rightarrow \mathfrak{g l}_{c}(V)$ onde $\mathfrak{I m} \rho_{c}=(\mathfrak{I m} \rho)_{c}$ e ker $\rho_{c}=(\mathfrak{k e r} \rho)_{c}$. Portanto, $\rho_{c}$ é sobrejetora (injetora) se e somente se $\rho$ é sobrejetora (injetora).

\section{Cohomologia de Álgebras de Lie}

Seja g uma álgebra de Lie (real), $V$ um espaço vetorial também real, ambos de dimensão finita, e $\rho: \mathfrak{g} \rightarrow \mathfrak{g l}(V)$ um homomorfismo de álgebras de Lie. é,

Definimos $H^{0}(\mathfrak{g}, V)_{\rho}$ como sendo o subespaço de $V$ que é anulado por $\rho$, isto

$$
H^{0}(\mathfrak{g}, V)_{\rho}=\{u \in V: \rho(X) u=0, \forall X \in \mathfrak{g}\} .
$$

Omitiremos o símbolo $\rho$ da notação a menos que seja necessario.

Lema 1.6 Se g é nilpotente, então pela Proposição 1.5, o Teorema 1.4 vale para a complexificação de $\rho$. Assumindo a notação do Teorema 1.4 Se existe $i \in\{1, \ldots, k\}$ tal que ker $h_{i}=\mathfrak{g}$, então $H^{0}(\mathfrak{g}, V) \neq\{0\}$. 
Prova. Desde que

$W_{i}=\left\{u \in V: 0=\left(\rho(X)-h_{i}(X) I\right)^{m} u=\rho(X)^{m} u, \forall X \in \mathfrak{g}\right.$ e algum $\left.m \in \mathbb{Z}\right\}$

$\rho$ age nilpotentemente sobre $W_{i}$. Logo Teorema de Engel garante a existência de um vetor não nulo $v \in W_{i}: \rho(X) v=0, \forall X \in \mathfrak{g}$. Portanto, $H^{0}(\mathfrak{g}, V) \neq\{0\}$.

Um 1-COCicLo é uma aplicação linear $f: \mathfrak{g} \rightarrow V$ tal que

$$
f([X, Y])=\rho(X) f(Y)-\rho(Y) f(X), \quad \forall X, Y \in \mathfrak{g} .
$$

O conjunto dos 1-cociclos forma um sub-espaço de $\operatorname{Hom}(\mathfrak{g}, V)$ o espaço vetorial das aplicações lineares de $\mathfrak{g}$ em $\mathrm{V}$. Denotaremos esse sub-espaço por $Z^{1}(\mathfrak{g}, V)$.

Um 1-COBORDo é um elemento $f$ de $Z^{1}(\mathfrak{g}, V)$ tal que existe $u \in V$ com a propriedade que

$$
f(X)=\rho(X) u, \quad \forall X \in \mathfrak{g} .
$$

O conjunto dos 1-cobordos forma um sub-espaço do espaço dos 1-cociclos e o denotaremos por $B^{1}(\mathfrak{g}, V)$.

Definimos $H^{1}(\mathfrak{g}, V)$ como o espaço quociente de $Z^{1}(\mathfrak{g}, V)$ por $B^{1}(\mathfrak{g}, V)$.

\section{Exemplos}

Açāo trivial. Seja $\rho$ uma ação trivial, isto é, $\rho(X) u=0, \forall X \in \mathfrak{g}$ e $\forall u \in V$. Da definição

$$
\begin{aligned}
Z^{1}(\mathfrak{g}, V) & =\{f \in \operatorname{Hom}(\mathfrak{g}, V): f([X, Y])=0, \forall X, Y \in \mathfrak{g}\} \quad \mathrm{e} \\
B^{1}(\mathfrak{g}, V) & =\{0\} .
\end{aligned}
$$

Portanto $H^{1}(\mathfrak{g}, V)=H o m(\mathfrak{g} /[\mathfrak{g}, \mathfrak{g}], V)$.

Concluimos então que o primeiro grupo de cohomologia depende da estruturada álgebra de Lie. Outra prova disso é dada pelo Primeiro Lema de Whitehead [6] que assegura que se a álgebra de Lie g é semi-simples, então $H^{1}(\mathfrak{g}, V)=0$, para qualquer $\rho$.

Açōes da álgebra de Lie 1-dimensional. Seja g 1-dimensional e X um gerador para $\mathfrak{g}$. Tome $f \in \operatorname{Hom}(\mathfrak{g}, V)$ e $\mathrm{R}, \mathrm{R} \in \mathfrak{g}, \mathrm{S}=\mathrm{sX}$ e $\mathrm{R}=\mathrm{rX}, \mathrm{s}, \mathrm{r} \in \mathbb{R}$. Então $[\mathrm{S}, \mathrm{R}]=[\mathrm{sX}, \mathrm{rX}]=0$ e $\rho(\mathrm{S}) f(\mathrm{R})-\rho(\mathrm{R}) f(\mathrm{~S})=\rho(\mathrm{sX}) f(\mathrm{rX})-\rho(\mathrm{rX}) f(\mathrm{sX})=$ 0 . Portanto $Z^{1}(\mathfrak{g}, V)=\operatorname{Hom}(\mathfrak{g}, V) \cong V$. Por outro lado, $B^{1}(\mathfrak{g}, V)=\{f \in$ $Z^{1}(\mathfrak{g}, V): \exists u \in V$ e $\left.f(W)=\rho(W) u, \forall W \in \mathfrak{g}\right\}$. Desde que $\mathfrak{g}$ é gerado por $\mathrm{X}$, $f$ fica determinado pelo seu valor em $\mathrm{X}$. $\operatorname{Logo} B^{1}(\mathfrak{g}, V) \cong \mathfrak{I m} \rho(\mathrm{X})$. Portanto $H^{1}(\mathfrak{g}, V) \cong \operatorname{Coker}(\rho(\mathrm{X}))$.

Com isso vemos que o primeiro grupo de cohomologia depende também ação. 
A. Ação da Álgebra de Lie $\mathfrak{r}^{2}$. Consideremos $\{X, Y\}$ base para $\mathfrak{r}^{2}$ e $\widetilde{X}(x, y)=$ $(x, 0)$ e $\tilde{Y}(x, y)=(0, y)$ campo de vetores em $\mathbb{R}^{2}$. Desde que esse dois campos comutam, isto é, $[\widetilde{X}, \tilde{Y}]=0$ podemos definir $\rho: \mathfrak{r}^{2} \rightarrow \Im\left(\mathbb{R}^{2}\right)$ uma ação tal que $\rho(X)=\widetilde{X}$ e $\rho(Y)=\widetilde{Y}$. Dado $W=(a, b) \in \mathfrak{r}^{2}$ e $u=\left(u_{1}, u_{2}\right) \in \mathbb{R}^{2}$, então

$$
\rho(W) u=(a \widetilde{X}+b \tilde{Y}) u=\left(a u_{1}, b u_{2}\right) .
$$

Logo, $H^{0}\left(\mathfrak{r}^{2}, \mathbb{R}^{2}\right)=\left\{u \in \mathbb{R}^{2}: \rho(W) u=0, \forall W \in \mathfrak{r}^{2}\right\}=\{0\}$. O espaço dos 1cociclos é formado pelas aplicações lineares de $\mathfrak{r}^{2}$ em $E^{2}$ tal que

$$
0=f([X, Y])=\widetilde{X} f(Y)-\tilde{Y} f(X) .
$$

Suponhamos que $f=\left(\begin{array}{cc}\alpha & \gamma \\ \beta & \delta\end{array}\right)$. Então $\widetilde{X} f(Y)=\tilde{Y} f(X) \Leftrightarrow(\gamma, 0)=(0, \beta)$. Portanto $Z^{1}\left(\mathfrak{r}^{2}, \mathbb{R}^{2}\right)=\{f: \beta, \gamma=0\}$. Desde que

$$
f(W)=\left(\begin{array}{cc}
\alpha & 0 \\
0 & \delta
\end{array}\right)\left(\begin{array}{c}
a \\
b
\end{array}\right)=\left(\begin{array}{c}
\alpha a \\
\delta b
\end{array}\right)
$$

então $f(W)=\rho(W) u, \forall W \in \mathfrak{r}^{2} \Leftrightarrow u_{1}=\alpha$ e $u_{2}=\delta$. Logo, para todo $f \in$ $Z^{1}\left(\mathfrak{r}^{2}, \mathbb{R}^{2}\right)$, existe $u=(\alpha, \delta) \in \mathbb{R}^{2}$ tal que $f(W)=\rho(W) u, \forall W \in \mathfrak{r}^{2}$. Portanto $H^{1}\left(\mathfrak{r}^{2}, \mathbb{R}^{2}\right)=\{0\}$.

B. Ação da Álgebra de Lie af. Consideremos $\varphi: A f \times \mathbb{R}^{2} \rightarrow \mathbb{R}^{2}$ a restrição da ação natural de $G L(2, n)$ sobre $\mathbb{R}^{2}$. Essa ação tem um ponto fixo. As outras órbitas são unidimensionais.

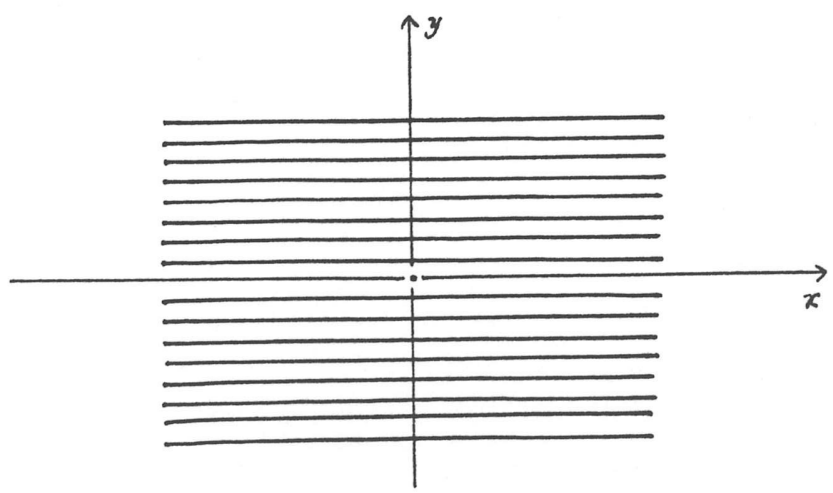

órbitas da ação $\varphi$ 
Fixe $p=(x, y) \in \mathbb{R}^{2}$ e consideremos $a, b$ como no cálculo de $\mathfrak{a} \mathfrak{f}, \mathrm{e}$

$$
\begin{aligned}
\varphi_{a}^{p}: \mathbb{R} & \rightarrow \mathbb{R}^{2} \\
& t \mapsto \varphi(a(t), p)=\left(x e^{t}, y\right) \\
\varphi_{b}^{p}: \mathbb{R} & \rightarrow \mathbb{R}^{2} \\
t & \mapsto \varphi(b(t), p)=(x+t y, y)
\end{aligned}
$$

Isso nos dá dois campos em $\mathbb{R}^{2}, \widetilde{X}$ e $\tilde{Y}$, a saber

$$
\begin{aligned}
& \widetilde{X}(x, y)=\left[\frac{\partial}{\partial t} \varphi_{a}^{p}\right]_{t=0}=(x, 0) \\
& \tilde{Y}(x, y)=\left[\frac{\partial}{\partial t} \varphi_{b}^{p}\right]_{t=0}=(y, 0)
\end{aligned}
$$

Temos portanto a ação induzida $\rho: \mathfrak{a} \mathfrak{f} \rightarrow \Im\left(\mathbb{R}^{2}\right)$ tal que $\rho(X)=\widetilde{X}, \rho(Y)=\tilde{Y}$. Seja $W=(r, s) \in$ af. Desde que

$$
\rho(W) p=(r \widetilde{X}+s \tilde{Y}) p=(r x+s y, 0)
$$

temos que $\rho(W) p=0 \forall W \in \mathfrak{a} \mathfrak{f} \Leftrightarrow x, y=0$. Portanto $H^{0}\left(\mathfrak{a} \mathfrak{f}, \mathbb{R}^{2}\right)=\{0\}$.

Sendo $\{X, Y\}$ base da álgebra de af,$Z^{1}\left(\mathfrak{a} \mathfrak{f}, \mathbb{R}^{2}\right)$ é o subespaço formado pelas $f \in \operatorname{Hom}\left(\mathfrak{a} \mathfrak{f}, \mathbb{R}^{2}\right)$ tal que

$$
f(Y)=\widetilde{X} f(Y)-\widetilde{Y} f(X)
$$

Suponha $f=\left[\begin{array}{ll}\alpha_{1} & \alpha_{2} \\ \beta_{1} & \beta_{2}\end{array}\right]$. Então,

$$
f(Y)=\widetilde{X} f(Y)-\widetilde{Y} f(X) \Leftrightarrow\left(\alpha_{2}, \beta_{2}\right)=\left(\alpha_{2}-\beta_{1}, 0\right) \Leftrightarrow \beta_{1}, \beta_{2}=0 .
$$

Logo, $Z^{1}\left(\mathfrak{a} \mathfrak{f}, \mathbb{R}^{2}\right)=\left\{f:=\beta_{1}, \beta_{2}=0\right\}$. Para calcular $B^{1}\left(\mathfrak{a} \mathfrak{f}, \mathbb{R}^{2}\right)$ tomemos $f \in Z^{1}\left(\mathfrak{a} \mathfrak{f}, \mathbb{R}^{2}\right)$. Desde que

$$
f(W)=\left[\begin{array}{cc}
\alpha_{1} & \alpha_{2} \\
0 & 0
\end{array}\right]\left[\begin{array}{l}
r \\
s
\end{array}\right]=\left[\begin{array}{c}
r \alpha_{1}+s \alpha_{2} \\
0
\end{array}\right]
$$

temos, $f(W)=\rho(W) p, \forall W \in$ af se e somente se

$$
r\left(\alpha_{1}-x\right)+s\left(\alpha_{2}-y\right)=0, \forall r, s \in \mathbb{R} \Leftrightarrow x=\alpha_{1}, y=\alpha_{2} .
$$

Logo, $\forall f \in Z^{1}$ (af, $\left.\mathbb{R}^{2}\right), \exists p=\left(\alpha_{1}, \alpha_{2}\right) \in \mathbb{R}^{2}$ tal que $f(W)=\rho(W) p, \forall W \in \mathfrak{a f}$.

Portanto, $H^{1}\left(\mathfrak{a f}, \mathbb{R}^{2}\right)=\{0\}$.

Exemplo C. Ação da Álgebra de Lie $\mathfrak{g}_{A}$. Consideremos $\varphi: G_{A} \times \mathbb{R}^{4} \rightarrow \mathbb{R}^{4}$ a restrição da ação natural de $G L(4, \mathbb{R})$ sobre $\mathbb{R}^{4}$. Essa ação tem $\{0\} \times \mathbb{R}^{3}$ como 
conjunto de pontos fixos. As outras órbitas são os planos $\{c\} \times \mathbb{R}^{3}, c$ real não nulo fixo.

Fixe $p=(x, y, z, w) \in \mathbb{R}^{4}$ e consideremos $a, b$ e $c$ como no cálculo de $\mathfrak{g}_{A}$ e

$$
\begin{aligned}
\varphi_{a}^{p}: \mathbb{R} & \rightarrow \mathbb{R}^{4} \\
t & \mapsto \varphi(a(t), p)=(x+t w, y, z, w) \\
\varphi_{b}^{p}: \mathbb{R} & \rightarrow \mathbb{R}^{4} \\
t & \mapsto \varphi(b(t), p)=(x, y+t w, z, w) \\
\varphi_{c}^{p}: \mathbb{R} & \rightarrow \mathbb{R}^{4} \\
t & \mapsto \varphi(c(t), p)=(x, y, z+t w, w)
\end{aligned}
$$

Isso nos dá três campos em $\mathbb{R}^{4}, \widetilde{X}, \tilde{Y}$ e $\widetilde{Z}$, a saber

$$
\begin{aligned}
\widetilde{X}(x, y, z, w) & =\left[\frac{\partial}{\partial t} \varphi_{a}^{p}\right]_{t=0}=(w, 0,0,0) \\
\tilde{Y}(x, y, z, w) & =\left[\frac{\partial}{\partial t} \varphi_{b}^{p}\right]_{t=0}=(0, w, 0,0) \\
\widetilde{Z}(x, y, z, w) & =\left[\frac{\partial}{\partial t} \varphi_{c}^{p}\right]_{t=0}=(0,0, w, 0)
\end{aligned}
$$

Temos portanto a ação induzida $\rho: \mathfrak{g}_{A} \rightarrow \Im\left(\mathbb{R}^{4}\right)$ tal que $\rho(X)=\widetilde{X}, \rho(Y)=$ $\tilde{Y}, \rho(Z)=\widetilde{Z}$. Seja $W=(r, s, l) \in \mathfrak{g}_{A}$. Desde que

$$
\rho(W) p=(r \widetilde{X}+s \tilde{Y}+l \widetilde{Z}) p=(r w, s w, l w, 0)
$$

temos que $\rho(W) p=0 \Leftrightarrow w=0$. Portanto $H^{0}\left(\mathfrak{g}_{A}, \mathbb{R}^{4}\right)=\mathbb{R}^{3} \times\{0\}$.

Sendo $\{X, Y, Z\}$ base da álgebra de Lie abeliana $\mathfrak{g}_{A}, Z^{1}\left(\mathfrak{g}_{A}, \mathbb{R}^{4}\right)$ é o subespaço formado pelas $f \in \operatorname{Hom}\left(\mathfrak{g}_{A}, \mathbb{R}^{4}\right)$ tal que

$$
\begin{gathered}
0=f([X, Y])=\widetilde{X} f(Y)-\tilde{Y} f(X) \\
0=f([X, Z])=\widetilde{X} f(Z)-\widetilde{Z} f(X) . \\
\text { Suponha } f=\left[\begin{array}{lll}
\alpha_{1} & \alpha_{2} & \alpha_{3} \\
\beta_{1} & \beta_{2} & \beta_{3} \\
\gamma_{1} & \gamma_{2} & \gamma_{3} \\
\delta_{1} & \delta_{2} & \delta_{3}
\end{array}\right] . \text { Logo, } \\
\left\{\begin{array} { l } 
{ 0 = \widetilde { X } f ( Y ) - \tilde { Y } f ( X ) = ( \delta _ { 9 , } , - \delta _ { 1 } , 0 , 0 ) } \\
{ 0 = \widetilde { X } f ( Z ) - \widetilde { Z } f ( X ) = ( \delta _ { 3 } , 0 , - \delta _ { 1 } , 0 ) } \\
{ 0 = \tilde { Y } f ( Z ) - \widetilde { Z } f ( Y ) = ( 0 , \delta _ { 3 } , - \delta _ { 2 } , 0 ) }
\end{array} \Leftrightarrow \left\{\begin{array}{l}
\delta_{2}=\delta_{1}=0 \\
\delta_{3}=\delta_{1}=0 . \\
\delta_{3}=\delta_{2}=0
\end{array}\right.\right.
\end{gathered}
$$


Portanto, $Z^{1}\left(\mathfrak{g}_{A}, \mathbb{R}^{4}\right)=\left\{f: \delta_{1}=\delta_{2}=\delta_{3}=0\right\}$. Para calcular $B^{1}\left(\mathfrak{g}_{A}, \mathbb{R}^{4}\right)$ tomemos $f \in Z^{1}\left(\mathfrak{g}_{A}, \mathbb{R}^{4}\right)$. Desde que

$$
f(W)=\left[\begin{array}{ccc}
\alpha_{1} & \alpha_{2} & \alpha_{3} \\
\beta_{1} & \beta_{2} & \beta_{3} \\
\gamma_{1} & \gamma_{2} & \gamma_{3} \\
0 & 0 & 0
\end{array}\right]\left[\begin{array}{l}
r \\
s \\
l
\end{array}\right]=\left[\begin{array}{c}
r \alpha_{1}+s \alpha_{2}+l \alpha_{3} \\
r \beta_{1}+s \beta_{2}+l \beta_{3} \\
r \gamma_{1}+s \gamma_{2}+l \gamma_{3} \\
0
\end{array}\right]
$$

temos, $f(W)=\rho(W) p, \forall W \in \mathfrak{g}_{A}$ se e somente se

$$
\left\{\begin{array}{c}
r\left(\alpha_{1}-w\right)+s \alpha_{2}+l \alpha_{3}=0 \\
r \beta_{1}+s\left(\beta_{2}-w\right)+l \beta_{3}=0 \\
r \gamma_{1}+s \gamma_{2}+l\left(\gamma_{3}-w\right)=0
\end{array}, \forall r, s, l \in \mathbb{R} \Leftrightarrow\left\{\begin{array}{c}
\alpha_{1}=w, \alpha_{2}=0, \alpha_{3}=0 \\
\beta_{1}=0, \beta_{2}=w, \beta_{3}=0 \\
\gamma_{1}=0, \gamma_{2}=0, \gamma_{3}=w
\end{array}\right.\right.
$$

Logo, $\forall f=\left[\begin{array}{ccc}\alpha_{1} & 0 & 0 \\ 0 & \alpha_{1} & 0 \\ 0 & 0 & \alpha_{1} \\ 0 & 0 & 0\end{array}\right] \in Z^{1}\left(\mathfrak{g}_{A}, \mathbb{R}^{4}\right), \exists p=\left(x, y, z, \alpha_{1}\right) \in \mathbb{R}^{4}$ tal que $f(W)=$ $\rho(W) p, \forall W \in \mathfrak{g}_{A}$.

Portanto, $H^{1}\left(\mathfrak{g}_{A}, \mathbb{R}^{4}\right)=\frac{Z^{1}\left(\mathfrak{g}_{A}, \mathbb{R}^{4}\right)}{B^{1}\left(\mathfrak{g}_{A}, \mathbb{R}^{4}\right)} \neq\{0\}$.

D. Ação da Álgebra de Lie $\mathfrak{g}_{N}$. Consideremos $\varphi: G_{N} \times \mathbb{R}^{3} \rightarrow \mathbb{R}^{3}$ a restrição da ação natural de $G L(3, \mathbb{R})$ sobre $\mathbb{R}^{3}$.

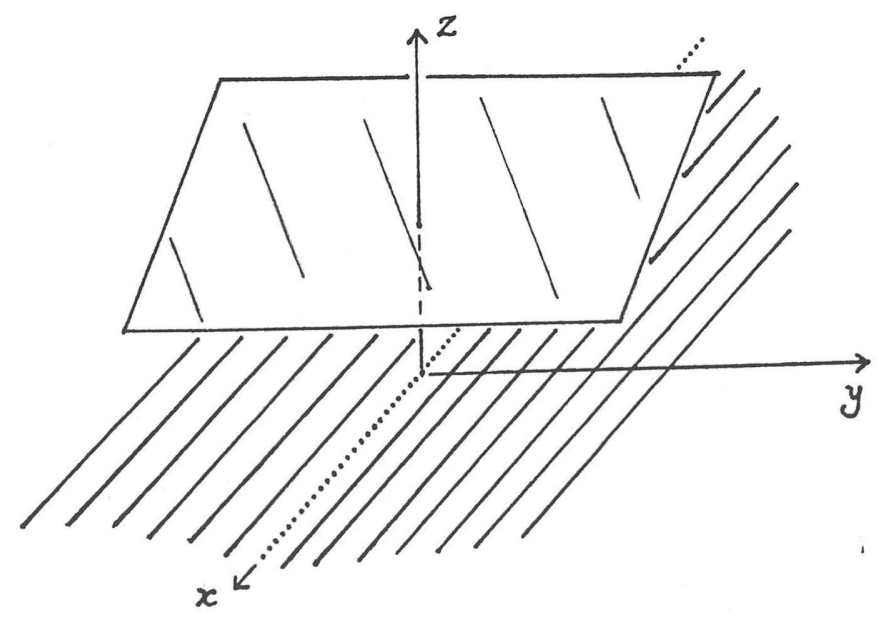

órbitas da ação $\varphi$

Fixe $p=(x, y, z) \in \mathbb{R}^{3}$ e consideremos $a, b$ e $c$ como no cálculo de $\mathfrak{g}_{N} \mathrm{e}$

$$
\begin{aligned}
\varphi_{a}^{p}: \mathbb{R} & \rightarrow \mathbb{R}^{3} \\
t & \mapsto \varphi(a(t), p)=(x+t y, y, z)
\end{aligned}
$$




$$
\begin{aligned}
\varphi_{b}^{p}: \mathbb{R} & \rightarrow \mathbb{R}^{3} \\
t & \mapsto \varphi(b(t), p)=(x, y+t z, z) \\
\varphi_{c}^{p}: \mathbb{R} & \rightarrow \mathbb{R}^{3} \\
t & \mapsto \varphi(c(t), p)=(x+t z, y, z)
\end{aligned}
$$

Isso nos dá três campos em $\mathbb{R}^{3}, \widetilde{X}, \widetilde{Y}$ e $\widetilde{Z}$, a saber

$$
\begin{aligned}
& \widetilde{X}(x, y, z)=\left[\frac{\partial}{\partial t} \varphi_{a}^{p}\right]_{t=0}=(y, 0,0) \\
& \tilde{Y}(x, y, z)=\left[\frac{\partial}{\partial t} \varphi_{b}^{p}\right]_{t=0}=(0, z, 0) \\
& \tilde{Z}(x, y, z)=\left[\frac{\partial}{\partial t} \varphi_{c}^{p}\right]_{t=0}=(z, 0,0)
\end{aligned}
$$

Temos portanto a ação induzida $\rho: \mathfrak{g}_{N} \rightarrow \Im\left(\mathbb{R}^{3}\right)$ é tal que $\rho(X)=\widetilde{X}, \rho(Y)=$ $\tilde{Y}, \rho(Z)=\widetilde{Z}$. Seja $W=(r, s, l) \in \mathfrak{g}_{N}$. Desde que

$$
\rho(W) p=(r \widetilde{X}+s \tilde{Y}+l \widetilde{Z}) p=(r y+l z, s z, 0)
$$

temos que $\rho(W) p=0, \forall W \Leftrightarrow y, z=0$. Portanto $H^{0}\left(\mathfrak{g}_{N}, \mathbb{R}^{3}\right)=\left\{(x, 0,0) \in \mathbb{R}^{3}\right\}$.

Lembrando que os elementos da base $\{X, Y, Z\}$ de $\mathfrak{g}_{N}$ satisfazem $[X, Y]=$ $Z,[X, Z]=[Y, Z]=0$, temos que $Z^{1}\left(\mathfrak{g}_{N}, \mathbb{R}^{3}\right)$ é o subespaço formado pelas aplicações $f \in \operatorname{Hom}\left(\mathfrak{g}_{N}, \mathbb{R}^{3}\right)$ tal que

$$
\begin{aligned}
f(Z) & =f([X, Y])=\widetilde{X} f(Y)-\tilde{Y} f(X) \\
0 & =f([X, Z])=\widetilde{X} f(Z)-\widetilde{Z} f(X) . \\
0 & =f([Y, Z])=\widetilde{Y} f(Z)-\widetilde{Z} f(Y)
\end{aligned}
$$

Suponha $f=\left[\begin{array}{ccc}\alpha_{1} & \alpha_{2} & \alpha_{3} \\ \beta_{1} & \beta_{2} & \beta_{3} \\ \gamma_{1} & \gamma_{2} & \gamma_{3}\end{array}\right]$. Logo,

$$
\left\{\begin{array} { c } 
{ ( \alpha _ { 3 } , \beta _ { 3 } , \gamma _ { 3 } ) = \widetilde { X } f ( Y ) - \tilde { Y } f ( X ) = ( \beta _ { 2 } , - \gamma _ { 1 } , 0 ) } \\
{ 0 = \widetilde { X } f ( Z ) - \widetilde { Z } f ( X ) = ( \beta _ { 3 } - \gamma _ { 1 } , 0 , 0 ) } \\
{ 0 = \tilde { Y } f ( Z ) - \tilde { Z } f ( Y ) = ( - \gamma _ { 2 } , \gamma _ { 3 } , 0 ) }
\end{array} \Leftrightarrow \left\{\begin{array}{l}
\beta_{3}=-\gamma_{1}, \alpha_{3}=\beta_{2} \\
\beta_{3}=\gamma_{1} \Rightarrow \beta_{3}, \gamma_{1}=0 . \\
\gamma_{3}, \gamma_{2}=0
\end{array}\right.\right.
$$

Portanto, $Z^{1}\left(\mathfrak{g}_{N}, \mathbb{R}^{3}\right)=\left\{f: \gamma_{1}, \gamma_{2}, \gamma_{3}, \beta_{3}=0, \beta_{2}=\alpha_{3}\right\}$. Para calcular $B^{1}\left(\mathfrak{g}_{N}, \mathbb{R}^{3}\right)$ tomemos $f \in Z^{1}\left(\mathfrak{g}_{N}, \mathbb{R}^{3}\right)$. Desde que

$$
f(W)=\left[\begin{array}{ccc}
\alpha_{1} & \alpha_{2} & \alpha_{3} \\
\beta_{1} & \alpha_{3} & 0 \\
0 & 0 & 0
\end{array}\right]\left[\begin{array}{l}
r \\
s \\
l
\end{array}\right]=\left[\begin{array}{c}
r \alpha_{1}+s \alpha_{2}+\imath \alpha_{3} \\
r \beta_{1}+s \alpha_{3} \\
0
\end{array}\right]
$$


temos, $f(W)=\rho(W) p, \forall W \in \mathfrak{g}_{N}$ se e somente se $\left\{\begin{array}{rl}r\left(\alpha_{1}-y\right)+s \alpha_{2}+l\left(\alpha_{3}-z\right)=0 \\ r \beta_{1}+s\left(\alpha_{3}-z\right)=0\end{array}, \forall r, s, l \in \mathbb{R} \Leftrightarrow\left\{\begin{array}{l}\alpha_{1}=y, \alpha_{2}=0, \alpha_{3}=z . \\ \beta_{1}=0, \alpha_{3}=z\end{array}\right.\right.$ Logo, $\forall f=\left[\begin{array}{ccc}\alpha_{1} & 0 & \alpha_{3} \\ 0 & \alpha_{3} & 0 \\ 0 & 0 & 0\end{array}\right] \in Z^{1}\left(\mathfrak{g}_{N}, \mathbb{R}^{3}\right), \exists p=\left(x, \alpha_{1}, \alpha_{3}\right) \in \mathbb{R}^{3}$ tal que $f(W)=$ $\rho(W) p, \forall W \in \mathfrak{g}_{N}$.

Portanto, $H^{1}\left(\mathfrak{g}_{N}, \mathbb{R}^{3}\right)=\frac{Z^{1}\left(\mathfrak{g}_{N}, \mathbb{R}^{3}\right)}{B^{1}\left(\mathfrak{g}_{N}, \mathbb{R}^{3}\right)} \neq\{0\}$.

E. Ação da Álgebra de Lie $\mathfrak{g}_{S}$. Consideremos $\varphi: G_{S} \times \mathbb{R}^{4} \rightarrow \mathbb{R}^{4}$ a restrição de ação natural de $G L(4, \mathbb{R})$ sobre $\mathbb{R}^{4}$

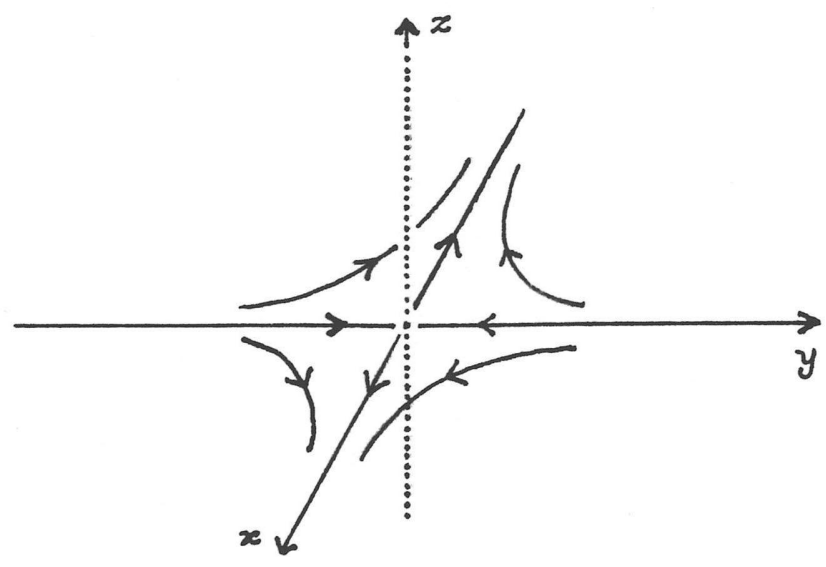

órbitas da ação $\varphi$ no subespaço $w=0$

Fixe $p=(x, y, z, w) \in \mathbb{R}^{4}$ e consideremos $a, b$ e $c$ como no cálculo de $\mathfrak{g}_{S}$ e

$$
\begin{aligned}
\varphi_{a}^{p}: \mathbb{R} & \rightarrow \mathbb{R}^{4} \\
t & \mapsto \varphi(a(t), p)=(x+t w, y, z, w) \\
\varphi_{b}^{p}: \mathbb{R} & \rightarrow \mathbb{R}^{4} \\
t & \mapsto \varphi(b(t), p)=(x, y+t w, z, w) \\
\varphi_{c}^{p}: \mathbb{R} & \rightarrow \mathbb{R}^{4} \\
t & \mapsto \varphi(c(t), p)=\left(x e^{t}, y e^{-t}, z+t w, w\right)
\end{aligned}
$$

Isso nos dá três campos em $\mathbb{R}^{4}, \widetilde{X}, \tilde{Y}$ e $\widetilde{Z}$, a saber

$$
\widetilde{X}(x, y, z, w)=\left[\frac{\partial}{\partial t} \varphi_{a}^{p}\right]_{t=0}=(w, 0,0,0)
$$




$$
\begin{aligned}
& \tilde{Y}(x, y, z, w)=\left[\frac{\partial}{\partial t} \varphi_{b}^{p}\right]_{t=0}=(0, w, 0,0) \\
& \widetilde{Z}(x, y, z, w)=\left[\frac{\partial}{\partial t} \varphi_{c}^{p}\right]_{t=0}=(x,-y, w, 0)
\end{aligned}
$$

Temos portanto a ação induzida $\rho: \mathfrak{g}_{S} \rightarrow \Im\left(\mathbb{R}^{4}\right)$ tal que $\rho(X)=\widetilde{X}, \rho(Y)=$ $\tilde{Y}, \rho(Z)=\widetilde{Z}$. Seja $W=(r, s, l) \in \mathfrak{g}_{S}$. Desde que

$$
\rho(W) p=(r \widetilde{X}+s \widetilde{Y}+l \tilde{Z}) p=(l x+r w, s w-l y, l w, 0)
$$

temos que $\rho(W) p=0, \forall W \Leftrightarrow x, y, w=0$. Logo $H^{0}\left(\mathfrak{g}_{S}, \mathbb{R}^{4}\right)=\left\{(0,0, z, 0) \in \mathbb{R}^{4}\right\}$.

Os elementos da base $\{X, Y, Z\}$ de $\mathfrak{g}_{S}$ satisfazem as seguintes relações: $[X, Y]=$ $0,[X, Z]=-X,[Y, Z]=Y$. Portanto, $Z^{1}\left(\mathfrak{g}_{S}, \mathbb{R}^{4}\right)$ é o subespaço formado pelas aplicações $f \in \operatorname{Hom}\left(\mathfrak{g}_{S}, \mathbb{R}^{4}\right)$ tal que

$$
\begin{aligned}
0 & =f([X, Y])=\widetilde{X} f(Y)-\tilde{Y} f(X) \\
-f(X) & =f([X, Z])=\widetilde{X} f(Z)-\widetilde{Z} f(X) \\
f(Y) & =f([Y, Z])=\widetilde{Y} f(Z)-\widetilde{Z} f(Y)
\end{aligned}
$$

Suponha $f=\left[\begin{array}{ccc}\alpha_{1} & \alpha_{2} & \alpha_{3} \\ \beta_{1} & \beta_{2} & \beta_{3} \\ \gamma_{1} & \gamma_{2} & \gamma_{3} \\ \delta_{1} & \delta_{2} & \delta_{3}\end{array}\right]$. Logo,

$\left\{\begin{array}{c}0=\widetilde{X} f(Y)-\tilde{Y} f(X)=\left(\delta_{2},-\delta_{1}, 0,0\right) \\ -\left(\alpha_{1}, \beta_{1}, \gamma_{1}, \delta_{1}\right)=\widetilde{X} f(Z)-\widetilde{Z} f(X)=\left(\delta_{3}-\alpha_{1}, \beta_{1},-\delta_{1}, 0\right) \\ \left(\alpha_{2}, \beta_{2}, \gamma_{2}, \delta_{2}\right)=\tilde{Y} f(Z)-\widetilde{Z} f(Y)=\left(-\alpha_{2}, \delta_{3}+\beta_{2},-\delta_{2}, 0\right)\end{array} \Leftrightarrow\left\{\begin{array}{l}\delta_{2}, \delta_{1}=0 \\ \gamma_{1}, \beta_{1}, \delta_{3}=0 . \\ \gamma_{2}, \delta_{3}, \alpha_{2}=0\end{array}\right.\right.$

Portanto, $Z^{1}\left(\mathfrak{g}_{S}, \mathbb{R}^{4}\right)=\left\{f: \delta_{1}, \delta_{2}, \delta_{3}, \gamma_{1}, \gamma_{2}, \beta_{1}, \alpha_{2}=0\right\}$. Para calcular $B^{1}\left(\mathfrak{g}_{S}, \mathbb{R}^{4}\right)$ tomemos $f \in Z^{1}\left(\mathfrak{g}_{S}, \mathbb{R}^{4}\right)$. Desde que

$$
f(W)=\left[\begin{array}{ccc}
\alpha_{1} & 0 & \alpha_{3} \\
0 & \beta_{2} & \beta_{3} \\
0 & 0 & \gamma_{3} \\
0 & 0 & 0
\end{array}\right]\left[\begin{array}{l}
r \\
s \\
l
\end{array}\right]=\left[\begin{array}{c}
r \alpha_{1}+l \alpha_{3} \\
s \beta_{2}+l \beta_{3} \\
l \gamma_{3} \\
0
\end{array}\right]
$$

temos, $f(W)=\rho(W) p, \forall W \in \mathfrak{g}_{S}$ se e somente se

$$
\left\{\begin{array}{c}
r\left(\alpha_{1}-w\right)+l\left(\alpha_{3}-x\right)=0 \\
s\left(\beta_{2}-w\right)+l\left(\beta_{3}+y\right)=0 \\
l\left(\gamma_{3}-w\right)=0
\end{array}, \forall r, s, l \in \mathbb{R} \Leftrightarrow\left\{\begin{array}{l}
\alpha_{1}=w, \alpha_{3}=x \\
\beta_{2}=w, \beta_{3}=-y . \\
\gamma_{3}=w
\end{array} .\right.\right.
$$


Logo, $\forall f=\left[\begin{array}{ccc}\alpha_{1} & 0 & \alpha_{3} \\ 0 & \alpha_{1} & \beta_{3} \\ 0 & 0 & \alpha_{1} \\ 0 & 0 & 0\end{array}\right] \in Z^{1}\left(\mathfrak{g}_{S}, \mathbb{R}^{4}\right), \exists p=\left(\frac{\alpha_{3}}{k}, \frac{-\beta_{3}}{k}, z, \alpha_{1}\right) \in \mathbb{R}^{4}$ tal que $f(W)=\rho(W) p, \forall W \in \mathfrak{g}_{\mathfrak{S}}$.

Portanto, $H^{1}\left(\mathfrak{g}_{S}, \mathbb{R}^{4}\right)=\frac{Z^{1}\left(\mathfrak{g}_{S}, \mathbb{R}^{4}\right)}{B^{1}\left(\mathfrak{g}_{S}, \mathbb{R}^{4}\right)} \neq\{0\}$.

F. Ação da Álgebra de Lie $\mathfrak{s}^{3}$. Seja $\varphi: S^{3} \times \mathbb{H} \rightarrow \mathbb{H}$ definida por $\varphi(u, p)=$ $u p u^{-1}$, como na página 4. Tomemos

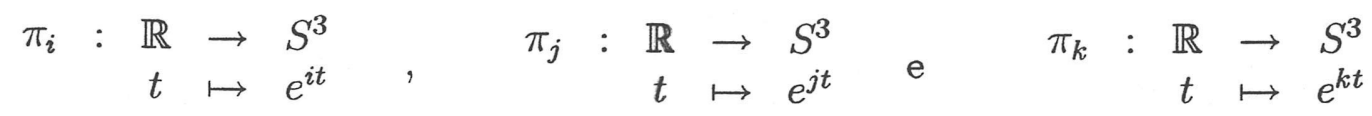

3 sub-grupos a 1-parâmetro. Seja $p=x+y i+z j+w k \in \mathbb{H}$. e defina as seguintes aplicações:

$$
\begin{aligned}
\varphi_{i}^{p}: \mathbb{R} & \rightarrow \mathbb{H} \\
t & \mapsto \varphi\left(\pi_{i}(t), p\right)=e^{i t} p e^{-i t}=(\cos (t)+\operatorname{sen}(t) i) p(\cos (t)-\operatorname{sen}(t) i) \\
\varphi_{j}^{p}: \mathbb{R} & \rightarrow \mathbb{H} \\
t & \mapsto \varphi\left(\pi_{j}(t), p\right)=e^{j t} p e^{-j t}=(\cos (t)+\operatorname{sen}(t) j) p(\cos (t)-\operatorname{sen}(t) j) \\
\varphi_{k}^{p}: \mathbb{R} & \rightarrow \mathbb{H} \\
t & \mapsto \varphi\left(\pi_{k}(t), p\right)=e^{k t} p e^{-k t}=(\cos (t)+\operatorname{sen}(t) k) p(\cos (t)-\operatorname{sen}(t) k)
\end{aligned}
$$

Então, simplificando obtemos

$$
\begin{aligned}
\varphi_{i}^{p}(t) & =x+y i+j(z \cos (2 t)-w \operatorname{sen}(2 t))+k(z \operatorname{sen}(2 t)+w \cos (2 t)) \\
\varphi_{j}^{p}(t) & =x+i(y \cos (2 t)+w \operatorname{sen}(2 t))+j z+k(w \cos (2 t)-y \operatorname{sen}(2 t)) \\
\varphi_{k}^{p}(t) & =x+i(y \cos (2 t)-z \operatorname{sen}(2 t))+j(y \operatorname{sen}(2 t)+z \cos (2 t))+k w
\end{aligned}
$$

Logo, derivando cada função em relação à $t$ quando $t=0$ obtemos os campos

$$
\begin{aligned}
\widetilde{X}(p) & =\left[\frac{\partial}{\partial t} \varphi_{i}^{p}\right]_{t=0}=[-2 j(z \operatorname{sen}(2 t)+w \cos (2 t))+2 k(z \cos (2 t)-w \operatorname{sen}(2 t))]_{t=0}= \\
& =-2 w j+2 z k \\
\tilde{Y}(p) & =\left[\frac{\partial}{\partial t} \varphi_{j}^{p}\right]_{t=0}=[2 i(w \cos (2 t)-y \operatorname{sen}(2 t))-2 k(w \operatorname{sen}(2 t)+y \cos (2 t))]_{t=0}= \\
& =2 w i-2 y k
\end{aligned}
$$




$$
\begin{aligned}
\widetilde{Z}(p) & =\left[\frac{\partial}{\partial t} \varphi_{k}^{p}\right]_{t=0}=[-2 i(y \operatorname{sen}(2 t)+z \cos (2 t))+2 j(y \cos (2 t)-z \operatorname{sen}(2 t))]_{t=0}= \\
& =-2 z i+2 y j
\end{aligned}
$$

Temos portanto a ação induzida $\rho: \mathfrak{s}^{3} \rightarrow \Im(\mathbb{H})$ tal que $\rho(X)=\frac{1}{2} \widetilde{X}, \rho(Y)=$ $\frac{1}{2} \tilde{Y}, \rho(Z)=\frac{1}{2} \widetilde{Z}$. Seja $W=(r, s, l) \in \mathfrak{s}^{3}$. Desde que

$$
\rho(W) p=(r \widetilde{X}+s \tilde{Y}+l \widetilde{Z}) p=(0, s w-r z, r y-l w, l z-s y)
$$

temos que $\rho(W) p=0, \forall W \Leftrightarrow y, z, w=0$. Portanto $H^{0}\left(\mathfrak{s}^{3}, \mathbb{H}\right)=\mathbb{R}$.

Como a base $\{X, Y, Z\}$ de $\mathfrak{s}^{3}$ satisfaz $[X, Y]=Z,[X, Z]=-Y,[Y, Z]=X$, temos que $Z^{1}\left(\mathfrak{s}^{3}, \mathbb{H}\right)$ é o subespaço formado pelas aplicações $f \in H o m\left(\mathfrak{s}^{3}, \mathbb{H}\right)$ tal que

$$
\begin{aligned}
& f(Z)=f([X, Y])=\widetilde{X} f(Y)-\tilde{Y} f(X) \\
& f(Y)=f([Z, X])=\widetilde{Z} f(X)-\widetilde{X} f(Z) \\
& f(X)=f([Y, Z])=\tilde{Y} f(Z)-\widetilde{Z} f(Y)
\end{aligned}
$$

Suponha $f=\left[\begin{array}{ccc}\alpha_{1} & \alpha_{2} & \alpha_{3} \\ \beta_{1} & \beta_{2} & \beta_{3} \\ \gamma_{1} & \gamma_{2} & \gamma_{3} \\ \delta_{1} & \delta_{2} & \delta_{3}\end{array}\right]$. Logo,

$$
\left\{\begin{array} { l } 
{ ( \alpha _ { 3 } , \beta _ { 3 } , \gamma _ { 3 } , \delta _ { 3 } ) = \widetilde { X } f ( Y ) - \tilde { Y } f ( X ) = ( 0 , - \delta _ { 1 } , - \delta _ { 2 } , \gamma _ { 2 } + \beta _ { 1 } ) } \\
{ ( \alpha _ { 2 } , \beta _ { 2 } , \gamma _ { 2 } , \delta _ { 2 } ) = \widetilde { Z } f ( X ) - \widetilde { X } f ( Z ) = ( 0 , - \gamma _ { 1 } , \beta _ { 1 } + \delta _ { 3 } , - \gamma _ { 3 } ) } \\
{ ( \alpha _ { 1 } , \beta _ { 1 } , \gamma _ { 1 } , \delta _ { 1 } ) = \widetilde { Y } f ( Z ) - \widetilde { Z } f ( Y ) = ( 0 , \delta _ { 3 } + \gamma _ { 2 } , - \beta _ { 2 } , - \beta _ { 3 } ) }
\end{array} \Leftrightarrow \left\{\begin{array}{l}
\beta_{3}=-\delta_{1}, \gamma_{3}=-\delta_{2} \\
\beta_{2}=-\gamma_{1}, \beta_{1}=0 . \\
\alpha_{3}, \alpha_{2}, \alpha_{1}, \gamma_{2}, \delta_{3}=0
\end{array}\right.\right.
$$

Portanto, $Z^{1}\left(\mathfrak{s}^{3}, \mathbb{H}\right)=\left\{f: \alpha_{1}, \alpha_{2}, \alpha_{3}, \beta_{1}, \gamma_{2}, \delta_{3}=0, \beta_{2}=-\gamma_{1}, \beta_{3}=-\delta_{1}, \delta_{2}=-\gamma_{3}\right\}$. Para calcular $B^{1}\left(\mathfrak{s}^{3}, \mathbb{H}\right)$ tomemos $f \in Z^{1}\left(\mathfrak{s}^{3}, \mathbb{H}\right)$. Desde que

$$
f(W)=\left[\begin{array}{ccc}
0 & 0 & 0 \\
0 & \beta_{2} & -\delta_{2} \\
-\beta_{2} & 0 & \gamma_{3} \\
\delta_{2} & -\gamma_{3} & 0
\end{array}\right]\left[\begin{array}{l}
r \\
s \\
l
\end{array}\right]=\left[\begin{array}{c}
0 \\
s \beta_{2}-l \delta_{2} \\
l \gamma_{3}-r \beta_{2} \\
r \delta_{2}-s \gamma_{3}
\end{array}\right]
$$

temos, $f(W)=\rho(W) p, \forall W \in \mathfrak{s}^{3}$ se e somente se

$$
\left\{\begin{array}{l}
s\left(\beta_{2}-w\right)+l\left(z-\delta_{2}\right)=0 \\
r\left(w-\beta_{2}\right)+l\left(\gamma_{3}-y\right)=0 \\
r\left(\delta_{2}-z\right)+s\left(y-\gamma_{3}\right)=0
\end{array}, \forall r, s, l \in \mathbb{R} \Leftrightarrow\left\{\begin{array}{l}
w=\beta_{2}, z=\delta_{2} \\
w=\beta_{2}, y=\gamma_{3} \\
z=\delta_{2}, y=\gamma_{3}
\end{array}\right.\right.
$$

Logo, $\forall f \in Z^{1}\left(\mathfrak{s}^{3}, \mathbb{H}\right), \exists p=\left(x, \gamma_{3}, \delta_{2}, \beta_{2}\right) \in \mathbb{H}$ tal que $f(W)=\rho(W) p, \forall W \in \mathfrak{s}^{3}$.

Logo, $Z^{1}\left(\mathfrak{s}^{3}, \mathbb{H}\right)=B^{1}\left(\mathfrak{s}^{3}, \mathbb{H}\right)$ e portanto $H^{1}\left(\mathfrak{s}^{3}, \mathbb{H}\right)=0$. 


\section{Capítulo 2}

\section{Zeros Elementares de Álgebras de Lie de Campos de Vetores}

Neste capítulo consideramos ainda $\mathfrak{g}$ uma álgebra de Lie, $V$ um espaço vetorial, ambos sobre os reais e de dimensão finita, e $\rho$ uma ação de $\mathfrak{g}$ em $V$.

\section{$\mathrm{O}$ Anulamento de $\mathbf{H}^{1}$ para Álgebras de Lie Nilpotentes}

Nesta seção veremos alguns resultados que ajudarão na compreensão do significado do anulamento do primeiro grupo de cohomologia para uma álgebra de Lie nilpotente.

Lema 2.1 Suponha que exista $Z \in \mathfrak{g}$ tal que $Z \in \mathfrak{K e r}$ ad e $\rho(Z)$ é inversível. Então $H^{1}(\mathfrak{g}, V)=\{0\}$. temos

Prova. Desde que $Z$ está no centro de $\mathfrak{g}$, para todo $X \in \mathfrak{g}$ e $f$ um 1-cociclo

$$
0=f([X, Z])=\rho(X) f(Z)-\rho(Z) f(X) \Rightarrow \rho(Z) f(X)=\rho(X) f(Z) .
$$

Tome $u$ igual a inversa de $\rho(Z)$ aplicada a $f(Z)$, isto é, $u=(\rho(Z))^{-1} f(Z) \in V$. Como

$$
\begin{aligned}
0 & =\rho([X, Z])=[\rho(X), \rho(Y)]=\rho(X) \rho(Z)-\rho(Z) \rho(X) \Rightarrow \\
& \Rightarrow(\rho(Z))^{-1} \rho(X)=\rho(X)(\rho(Z))^{-1} .
\end{aligned}
$$

Então,

$f(X)=(\rho(Z))^{-1}(\rho(X) f(Z))=\rho(X)\left((\rho(Z))^{-1} f(Z)\right)=\rho(X) u$, para todo $X \in \mathfrak{g}$.

Logo $f$ é também um 1-cobordo e portanto $H^{1}(\mathfrak{g}, V)=\{0\}$.

Observaçāo. Se $\rho: \mathfrak{g} \rightarrow \mathfrak{g l}(V)$ é uma ação tal que $\mathfrak{k e r} \rho \neq\{0\}$, então $\rho$ induz uma aplicação

$$
\rho^{\prime}: \frac{\mathfrak{g}}{\mathfrak{K e r} \rho} \longrightarrow \mathfrak{g l}(V)
$$

dada por $\rho^{\prime}(\bar{X})=\rho(X)$, onde $\bar{X}$ é a classe de equivalência de $X$. Verifiquemos que $\rho^{\prime}$ é uma ação injetora. 
- $\rho^{\prime}$ é uma ação pois

$$
\left[\rho^{\prime}(\bar{X}), \rho^{\prime}(\bar{Y})\right]=[\rho(X), \rho(Y)]=\rho([X, Y])=\rho^{\prime}(\overline{X, Y})=\rho^{\prime}([\bar{X}, \bar{Y}]) .
$$

onde a útima igualdade é dada pela definição do colchete em $\mathfrak{g} / \mathfrak{K e r} \rho$ induzido do colchete de $\mathfrak{g}$ (em 1.3, pg.11).

- $\rho^{\prime}$ é injetora pois

$$
\rho^{\prime}(\bar{X})=0 \Leftrightarrow \rho(X)=0 \Leftrightarrow X \in \mathfrak{K e r} \rho \Leftrightarrow \bar{X}=0 .
$$

Lema 2.2 Se $\rho: \mathfrak{g} \rightarrow \mathfrak{g l}(V)$ é uma nil representação não trivial de $\mathfrak{g}$, então $H^{1}(\mathfrak{g}, V) \neq\{0\}$.

Prova. Se $\operatorname{dim} V=1$, o lema é válido por vacuidade. Suponha que $\operatorname{dim} V>$ 1 e $\operatorname{dim} \mathfrak{g}=1$. Como visto no exemplo Açāo Trivial, $H^{1}(\mathfrak{g}, V)=V / \mathfrak{I m} \rho(X)$ onde $X$ é um gerador de g. Logo, o lema também é válido pois $\operatorname{dim} V>1$ e $\operatorname{dim} \mathfrak{I m} \rho(X)=1$.

Suponhamos por um momento que já temos provado o Lema para $\mathfrak{g}^{\prime}=$ $\mathfrak{g} / \mathfrak{K e r} \rho$. Neste caso $H^{0}(\mathfrak{K e r} \rho, V)=V$. Então a Proposição 3.4 (pg.45) nos fornece a seguinte sequência exata:

$$
0 \longrightarrow H^{1}\left(\mathfrak{g}^{\prime}, V\right) \stackrel{\text { inf }}{\longrightarrow} H^{1}(\mathfrak{g}, V) \text {. }
$$

Logo $H^{1}(\mathfrak{g}, V) \neq\{0\}$ pois $H^{1}\left(\mathfrak{g}^{\prime}, V\right) \neq\{0\}$ por hipótese. Podemos então supor que $\rho$ é injetora e $\mathfrak{g}$ é nilpotente.

Suponhamos então que $\operatorname{dim} \mathfrak{g} \geq 2$ e que o resultado é verdadeiro para $\operatorname{dim} V=$ $n-1(n \geq 2)$. Seja $\left\{e_{1}, \ldots, e_{n}\right\}$ base para $V$, e $V_{0}$ o subespaço de $V$ gerado por $\left\{e_{1}, \ldots, e_{n-1}\right\}$. Logo $V / V_{0} \simeq \mathbb{R}$. Seja $\mathfrak{h} \subset \mathfrak{g}$ o ideal consistindo de todos os elementos de $\mathfrak{g}$ cuja restrição à $V_{0}$ é zero. Desde que $\rho$ é injetiva, $H^{0}\left(\mathfrak{h}, V_{0}\right)=V_{0}$. Temos então dois caso a analizar.

1. $\mathfrak{h} \neq \mathfrak{g}$. Então a Proposição 3.4 diz que

$$
0 \longrightarrow H^{1}\left(\mathfrak{g} / \mathfrak{h}, V_{0}\right) \longrightarrow H^{1}(\mathfrak{g}, V)
$$

é uma sequência exata e o Lema é verdadeiro pois o termo do meio é não nulo por hipótese.

2. $\mathfrak{h}=\mathfrak{g}$. Pela definição de $V_{0}, \mathfrak{g}$ age trivialmente sobre $V_{0}$ e a sequência exata curta

$$
0 \longrightarrow V_{0} \stackrel{\mu}{\longrightarrow} V \longrightarrow \mathbb{R} \longrightarrow 0
$$

produz, por 3.2 pg.44, a seguinte sequencia exata

$$
H^{0}(\mathfrak{g}, \mathbb{R}) \rightarrow H^{1}\left(\mathfrak{g}, V_{0}\right) \stackrel{\mu_{*}}{\rightarrow} H^{1}(\mathfrak{g}, V) .
$$


Como $\mathfrak{g}$ age trivialmente sobre $V_{0}$, então $H^{1}\left(\mathfrak{g}, V_{0}\right)=H o m\left(\mathfrak{g} /[\mathfrak{g}, \mathfrak{g}], V_{0}\right)$. Pelo Lema 1.3, pg.13, $\operatorname{dim} H o m\left(\mathfrak{g} /[\mathfrak{g}, \mathfrak{g}], V_{0}\right) \geq 2$. Desde que $H^{0}(\mathfrak{g}, \mathbb{R})$ é um subespaço de $\mathbb{R}$, então $\operatorname{dim} H^{0}(\mathfrak{g}, \mathbb{R}) \leq 1$. Como a sequência acima é exata, o Teorema do Núcleo e da Imagem garante que $\operatorname{dim} \mathfrak{I m} \mu_{*} \geq 1$, e a prova está completa.

Lema 2.3 Seja $\rho: \mathfrak{g} \rightarrow \mathfrak{g l}(V)$ uma ação e $V_{n}$ o maior subespaço de $V$ tal que todo elemento de $\rho(\mathfrak{g})$ é nilpotente. Então $H^{0}\left(\mathfrak{g}, V / V_{n}\right)=\{0\}$.

Prova. A ação $\rho$ induz uma ação $\bar{\rho}: \mathfrak{g} \rightarrow \mathfrak{g l}\left(V / V_{n}\right): X \mapsto \pi \circ \rho(X) \circ i$ onde $i$ é a aplicação tal que $i([u])=u$ e $\pi$ é a projeção canônica $\pi: V \rightarrow V / V_{n}: u \mapsto[u]$. Tome $v, u \in V$ tal que $(v-u) \in V_{n}$. Da definição de $V_{n}, \rho(X)(v-u) \in V_{n}$. Logo

$$
\begin{aligned}
\bar{\rho}(X)[v]-\bar{\rho}(X)[u] & =\pi \circ \rho(X) \circ i([v])-\pi \circ \rho(X) \circ i([u])= \\
& =\pi \circ \rho(X) v-\pi \circ \rho(X) u= \\
& =\pi(\rho(X)(v-u))=0 .
\end{aligned}
$$

Logo, $\bar{\rho}$ está bem definida. Além disso,

$$
\begin{aligned}
{[\bar{\rho}(X), \bar{\rho}(Y)] } & =\bar{\rho}(X) \bar{\rho}(Y)-\bar{\rho}(Y) \bar{\rho}(X)= \\
& =\pi \rho(X) \rho(Y) i-\pi \rho(Y) \rho(X) i= \\
& =\pi(\rho(X) \rho(Y)-\rho(Y) \rho(X)) i= \\
& =\pi(\rho([X, Y])) i= \\
& =\bar{\rho}([X, Y]) .
\end{aligned}
$$

Portanto $\bar{\rho}$ é uma ação de $\mathfrak{g}$ em $V / V_{n}$. Desde que $\bar{\rho}(X)[v]=0 \Leftrightarrow \pi \circ \rho(X) \circ i([v])=$ $\pi \circ \rho(X) v=0$, então $H^{0}\left(\mathfrak{g}, V / V_{n}\right)$ é o subespaço de $V / V_{n}$ tal que $\rho(X) v \in V_{n}, \forall X \in$ g. Mas pela definição de $V_{n}, v \in V_{n}$. Portanto $H^{0}\left(\mathfrak{g}, V / V_{n}\right)=\{0\}$.

Proposiçāo 2.4 Se $\mathfrak{g}$ é uma álgebra de Lie tal que $[\mathfrak{g}, \mathfrak{g}] \neq \mathfrak{g}$ e $\rho: \mathfrak{g} \rightarrow \mathfrak{g l}(V)$ é uma ação tal que $H^{1}(\mathfrak{g}, V)=\{0\}$, então $H^{0}(\mathfrak{g}, V)=\{0\}$.

Prova. Considere $V_{n}$ como no Lema anterior. Logo $H^{0}\left(\mathfrak{g}, V / V_{n}\right)=\{0\}$ e a sequência exata curta

$$
0 \rightarrow V_{n} \rightarrow V \rightarrow V / V_{n} \rightarrow 0
$$

produz, por 3.2 pg.44, a seguinte sequência exata de cohomologia

$$
H^{0}\left(\mathfrak{g}, V / V_{n}\right) \rightarrow H^{1}\left(\mathfrak{g}, V_{n}\right) \rightarrow H^{1}(\mathfrak{g}, V) .
$$

Desde que $H^{1}(\mathfrak{g}, V)=\{0\}$ por hipótese, então $H^{1}\left(\mathfrak{g}, V_{n}\right)=\{0\}$. Por outro lado, $H^{1}(\mathfrak{g}, V)=\{0\}$ e o Lema 2.2 implica que $\rho$ é trivial sobre $V_{n}$. Logo $H^{1}\left(\mathfrak{g}, V_{n}\right)=$ $\operatorname{Hom}\left(\mathfrak{g} /[\mathfrak{g}, \mathfrak{g}], V_{n}\right)$. Portanto $V_{n}=\{0\}$. Pela definição $H^{\mathbf{0}}(\mathfrak{g}, V) \subset V_{n}$ concluindo assim a Proposição. 
Por "Quase Todos" os pontos em um espaço vetorial real $V$ de dimensão finita entendemos o subconjunto $\left(V-\bigcup_{i=1}^{k} W_{i}\right)$ onde $k$ é finito e $W_{i}$ é um subespaço de $V$ tal que $\operatorname{dim} W_{i}<\operatorname{dim} V$.

Proposiçāo 2.5 Para uma álgebra de Lie nilpotente e uma ação $\rho: \mathfrak{g} \rightarrow \mathfrak{g l}(V)$ as seguintes afirmações são equivalentes:

(a) $H^{1}(\mathfrak{g}, V)=\{0\}$

(b) $H^{0}(\mathfrak{g}, V)=\{0\}$

(c) Para quase todo $X \in \mathfrak{g}, \rho(X)$ é inversível

(d) Para algum $X \in \mathfrak{g}, \rho(X)$ é inversível

Prova. Que (a) implica (b) segue da Proposição 2.4, (c) implica (d) é óbvio, (d) implica (b) segue da definição de $H^{0}$ e (b) implica (c) segue do Lema 1.6, pg.14, e do Teorema 1.4 pois as aplicações $h_{i}$ são tais que $\mathfrak{k e r} h_{i}$ é um subespaço fechado com $\operatorname{dim} \mathfrak{K e r} h_{i}<\operatorname{dim} \mathfrak{g}$. Logo, se $X \in\left(\mathfrak{g}-\bigcup_{i=1}^{k} \mathfrak{K e r} h_{i}\right)$, então $\rho(X)$ tem todos os autovalores não nulos, ou seja, $\rho(X)$ é inversível.

A prova estará completa se mostrarmos que (b) implica (a). Desde que (b) é equivalente à (c), pelo Lema 2.1 a Proposição é válida se g é abeliano. Em particular é verdadeira $\mathrm{p} / \operatorname{dim} \mathfrak{g} \leq 2$. Assuma que a Proposição vale para $\operatorname{dim} \mathfrak{g}<$ $n(n \geq 3)$. Se $\operatorname{dim} \mathfrak{g}=n$, pelo Lema 1.3 pg.13, e por (c) existe um ideal $\mathfrak{h} \subset \mathfrak{g}$ de codimensão 1 que contém um elemento inversível. Pela hipótese de indução, $H^{1}(\mathfrak{h}, V)=\{0\}$ e $H^{0}(\mathfrak{h}, V)=\{0\}$. A Proposição 3.4 (pg.45) diz que a sequência

$$
0 \longrightarrow H^{1}\left(\mathfrak{g} / \mathfrak{h}, H^{0}(\mathfrak{h}, V)\right) \stackrel{\text { inf }}{\longrightarrow} H^{1}(\mathfrak{g}, V) \stackrel{\text { res }}{\longrightarrow} H^{1}(\mathfrak{h}, V)
$$

é exata. Logo, $H^{1}(\mathfrak{g}, V)=\{0\}$, e a prova de 2.5 está completa.

\section{Zeros Elementares}

Um campo vetorial $X$, sobre uma variedade diferenciável $M$, é uma correspondência que associa a cada ponto $p \in M$ um vetor $X(p) \in T_{p} M$, tangente a $M$ no ponto $p$. Dado um campo vetorial $X$ sobre $M$, um sistema de coordenadas $\mathbf{x}: U \rightarrow \mathbb{R}^{k}$ em $M$ permite escrever, para todo $p \in U$ :

$$
X(p)=\sum_{i=1}^{k} a_{i}(p) \frac{\partial}{\partial \mathbf{x}_{i}}(p) .
$$

Assim, em cada sistema de coordenadas $\mathbf{x}$, o campo $X$ fica determinado pelas $n$ funções reais $a_{i}: U \rightarrow \mathbb{R}$ que dão, em todo ponto $p \in U$, as $n$ coordenadas de $X(p)$ relativa a base $\left\{\frac{\partial}{\partial \mathbf{x}_{i}}(p)\right\}$. Um ponto $p \in M$ é um ZERo de $X$ se $X(p)$ é o vetor nulo. Um zero de $X$ é dito ser Elementar se $D X_{p}: T_{p} M \rightarrow T_{p} M$ é inversível, isto é, $\operatorname{det}\left(\frac{\partial a_{i}}{\partial \mathbf{x}_{j}}\right) \neq 0$ no ponto $p$. 
Outra maneira de escrevermos esses conceitos é definir um campo vetorial como uma aplicação $X: M \rightarrow T M$ de classe $C^{r}$ tal que $\pi X$ é a identidade em $M$, onde $\pi$ é a projeção natural de $T M$ em $M$. Consideremos $M_{0}=\left\{0_{q} \in T_{q} M: q \in M\right\}$. $M_{0}$ é uma subvariedade de $T M$ difeomorfa à $M$. Neste caso $p \in M$ é um zero de $X$ se $X(p) \in M_{0}$. Além disso $p$ é um zero elementar de $X$ se $X$ é transversal à $M_{0}$ em $p$. Essa definição implica que todo zero elementar é isolado e estável, isto é, persistem por pequenas perturbações.

Seja $\Im^{r}(M)$ a álgebra de Lie dos campos vetoriais $C^{r}$ sobre $M$, e $\mathfrak{g}$ uma álgebra de Lie. Uma Ação de g sobre $M$ é uma aplicação linear $A: \mathfrak{g} \rightarrow \Im^{r}(M)$ tal que $A([X, Y])=[A(X), A(Y)]$, para todo $X, Y$ em $\mathfrak{g}$. Um ponto $p \in M$ é dito ser um ZERo de $A$ se $A(X)(p)=0$, para todo $X \in \mathfrak{g}$. Logo, se $A$ for integrado para obtermos uma ação local de um grupo de Lie $G$, então $p$ é um ponto estacionário (ou fixo) da ação local de $G$ [13]. Em particular, se $M$ é compacto temos uma ação global de $G$ sobre $M$. Por uma ÓrBita da ação $A$ entendemos uma órbita da ação do grupo de Lie.

Para cada $X \in \mathfrak{g}$ sua imagem por $A$ é um campo de vetores $A(X): M \rightarrow T M$, e a derivada de $A(X)$ em $p \in M$ é a aplicação linear

$$
D A(X)_{p}: T_{p} M \rightarrow T_{v_{p}}(T M), \quad \text { onde } v_{p}=A(X) p .
$$

Se p é um zero de $A$, então identificando $T_{p} M$ com a componente vertical de $T_{0_{p}}(T M)$ (onde $0_{p}$ é o vetor nulo em $p$ ) podemos considerar $D A(X)_{p} \in \mathfrak{g l}\left(T_{p} M\right)$. Logo, a aplicação

$$
\begin{aligned}
\rho: \mathfrak{g} & \rightarrow \mathfrak{g l}\left(T_{p} M\right) \\
X & \mapsto D A(X)_{p}
\end{aligned}
$$

é uma ação linear do tipo considerado anteriormente, e é chamada PARTE LINEAR de $A$ em $p$.

Definiçāo 2.6 Um zero $p \in M$ de uma ação A é chamado ElEMENTAR se, para a parte linear de $A$ em $p, H^{1}\left(\mathfrak{g}, T_{p} M\right)=\{0\}$.

A Proposição 2.5 nos fornece uma caracterização dos zeros elementares de uma ação de uma álgebra de Lie nilpotente.

Proposiçāo 2.7 Se g é nilpotente e p é um zero de uma ação $A: \mathfrak{g} \rightarrow \Im^{1}(M)$, então as seguintes afirmações são equivalentes:

(a) $p$ é um zero elementar para $A$;

(b) Se $D A(X)_{p} v=0$ para todo $X \in \mathfrak{g}$, então $v=0$;

(c) Para algum $X \in \mathfrak{g}, p$ é um zero elementar para $A(X)$;

(d) Para quase todo $X \in \mathfrak{g}, p$ é um zero elementar para $A(X)$. 
Prova. Segue imediatamente de 2.5.

Através dessa caracterização vemos facilmente que a ação de $\mathfrak{r}^{2}$ do exemplo A, página 15 , tem a origem como um zero elementar uma vez que o campo $\widetilde{W}=$ $a \widetilde{X}+b \widetilde{Y}$, com $a \neq 0$ e $b \neq 0$, é inversível. Por outro lado a ação de af do exemplo B nos mostra que a condição (c) não é necessaria para que um zero seja elementar para uma ação de uma álgebra de Lie não nilpotente. Já no caso de uma ação de uma álgebra de Lie semi-simples o Primeiro Lema de Whitehead diz que todo zero é elementar. Percebemos então que quanto menos comutativa é a álgebra de Lie mais fácil fica para um zero ser elementar.

A Proposição 2.5 nos fornece outra ferramenta para saber se um zero de uma ação $A: \mathfrak{g} \rightarrow \Im^{1}(M)$ é elementar.

Exemplo G. Considere os seguintes campos de vetores sobre $\mathbb{R}^{2}$ :

$$
\begin{aligned}
X & =\cos 2 \pi x \frac{\partial}{\partial x}+\cos 2 \pi y \frac{\partial}{\partial y} \\
Y & =\cos 2 \pi x \frac{\partial}{\partial x}-\cos 2 \pi y \frac{\partial}{\partial y}
\end{aligned}
$$

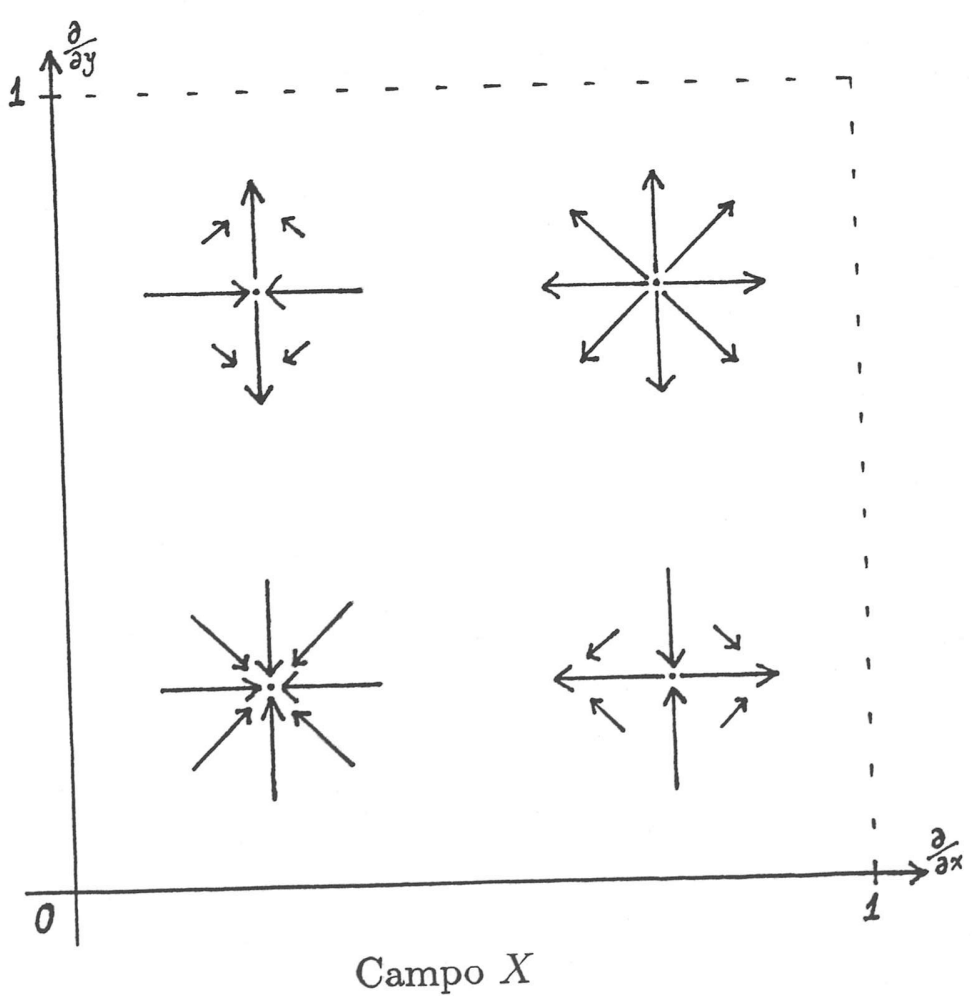

Esses dois campos de vetores comutam e definem outros dois campos de vetores $\widetilde{X}$ e $\widetilde{Y}$ sobre o toro $T^{2}=\mathbb{R}^{2} / \mathbb{Z}^{2}$. Tomemos então a ação $A$ expandida por $\widetilde{X}$ e $\tilde{Y}$. O conjunto de zeros dessa ação é $\left\{\left(\frac{1}{4}, \frac{1}{4}\right),\left(\frac{1}{4}, \frac{3}{4}\right),\left(\frac{3}{4}, \frac{1}{4}\right),\left(\frac{3}{4}, \frac{3}{4}\right)\right\}$. O módulo 
do determinante da matriz Jacobiana de $X$ em cada zero é $4 \pi^{2}$. Portanto todos os zeros de $A$ são elementares.

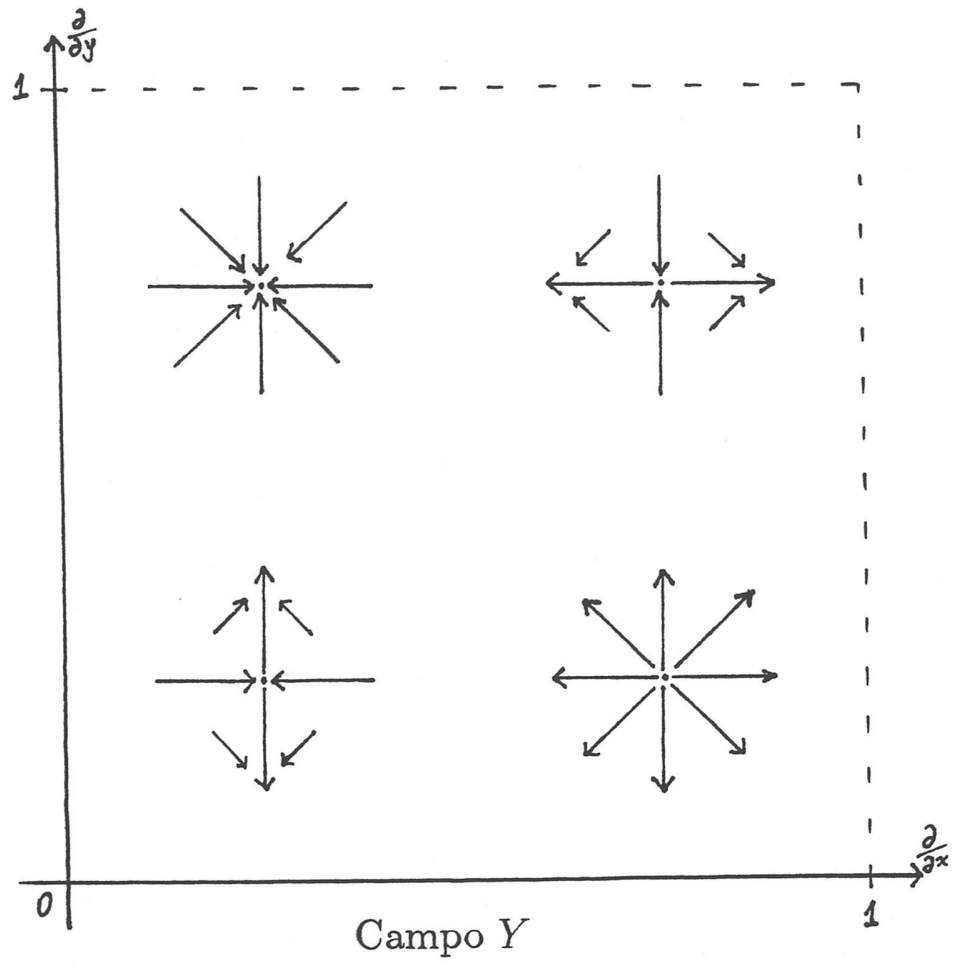

\section{Teorema de Estabilidade}

Consideremos para cada $u \in V$, a aplicação linear de $\mathfrak{g}$ em $V$ tal que

$$
\alpha_{u}(X)=\rho(X) u \text {. }
$$

Vamos agora definir uma aplicação linear $\alpha$ como sendo a avaliação da ação $\rho$, isto é,

$$
\begin{aligned}
\alpha: \quad V & \rightarrow \operatorname{Hom}(\mathfrak{g}, V) \\
u & \mapsto \alpha_{u}
\end{aligned}
$$

Da definição fica claro que $H^{0}(\mathfrak{g}, V)=\mathfrak{K} \mathfrak{e r} \alpha$.

Sejam $\rho$ e $\rho^{\prime}$ duas ações e $\alpha$ e $\alpha^{\prime}$ as corrrespondentes aplicações lineares. Se $\rho$ está próxima de $\rho^{\prime}$ então $\alpha$ está próxima de $\alpha^{\prime}$ e portanto, pela dependência contínua dos autovalores, dado $\rho$, existe $\rho^{\prime}$ suficientemente próximo de $\rho$ tal que

$$
\operatorname{dim} \mathfrak{I m} \alpha \leq \operatorname{dim} \mathfrak{I m} \alpha^{\prime} .
$$

Considere agora $W$ o espaço vetorial das aplicações bilineares anti-simétricas de $\mathfrak{g} \times \mathfrak{g}$ em $V$. Seja $l=\operatorname{dim} \mathfrak{g}$, então $W \simeq V^{\frac{l(l-1)}{2}}$. Então, para cada $f \in$ $\operatorname{Hom}(\mathfrak{g}, V)$ defina $\beta_{f}$ a aplicação bilinear de $\mathfrak{g} \times \mathfrak{g}$ em $V$ tal que

$$
\beta_{f}(X, Y)=\rho(X) f(Y)-\rho(Y) f(X)-f([X, Y]) .
$$


Claramente $\beta_{f}$ é anti-simétrica, isto é, $\beta_{f} \in W$. Portanto, podemos definir

$$
\begin{aligned}
\beta: H o m(\mathfrak{g}, V) & \rightarrow W \\
f & \longmapsto \beta_{f}
\end{aligned} .
$$

Da definição de $\beta$ segue que $Z^{1}(\mathfrak{g}, V)=\mathfrak{K e r} \beta$. Tome $u \in V$. Então

$$
\begin{aligned}
\beta\left(\alpha_{u}\right)(X, Y) & =\rho(X) \alpha_{u}(Y)-\rho(Y) \alpha_{u}(X)-\alpha_{u}([X, Y])= \\
& =\rho(X)(\rho(Y) u)-\rho(Y)(\rho(X) u)-(\rho(X) \rho(Y)-\rho(Y) \rho(X)) u= \\
& =0 .
\end{aligned}
$$

Logo $\operatorname{Im} \alpha \subset \mathfrak{K e r} \beta$. Mais dos que isso, seja $f \in \mathfrak{K e r} \beta=Z^{1}(\mathfrak{g}, V)$. Então

$$
f \in B^{1}(\mathfrak{g}, V) \Leftrightarrow \exists u \in V: f(X)=\rho(X) u=\alpha_{u}(X), \forall X \in \mathfrak{g},
$$

isto é, $f=\alpha_{u}$. Logo, $B^{1}(\mathfrak{g}, V)=\mathfrak{I m} \alpha$. Portanto

$$
H^{1}(\mathfrak{g}, V)=\frac{\mathfrak{K e r} \beta}{\mathfrak{I m} \alpha} .
$$

Consequentemente dizer que $H^{1}(\mathfrak{g}, V)=\{0\}$ é equivalente a dizer que a sequência

$$
V \stackrel{\alpha}{\longrightarrow} \operatorname{Hom}(\mathfrak{g}, V) \stackrel{\beta}{\longrightarrow} W
$$

é exata.

Analogamente ao dito sobre $\alpha$, dado uma ação $\rho$ existe $\rho^{\prime}$ ação suficientemente próxima de $\rho$ tal que, para as aplicações $\beta$ e $\beta^{\prime}$ correspondentes

$$
\operatorname{dim} \mathfrak{K e r} \beta^{\prime} \leq \operatorname{dim} \mathfrak{K e r} \beta
$$

Por 2.2, 2.4 e 2.6 temos que dada uma ação $\rho$, existe uma ação $\rho^{\prime}$ suficientemente próxima de $\rho$ tal que

$$
\operatorname{dim} \mathfrak{I m} \alpha \leq \operatorname{dim} \mathfrak{I m} \alpha^{\prime} \leq \operatorname{dim} \mathfrak{K e r} \beta^{\prime} \leq \operatorname{dim} \mathfrak{K e r} \beta .
$$

Se $H^{1}(\mathfrak{g}, V)_{\rho}=\{0\}$, isto é, $\mathfrak{I m} \alpha=\mathfrak{K e r} \beta$, então $H^{1}(\mathfrak{g}, V)_{\rho^{\prime}}=\{0\}$, ou seja, a trivialidade de $H^{1}$ é estável por pequenas perturbações da ação. Além disso, $\operatorname{dim} H^{0}(\mathfrak{g}, V)_{\rho}=\mathfrak{K e r} \alpha=\mathfrak{K e r} \alpha^{\prime}=\operatorname{dim} H^{0}(\mathfrak{g}, V)_{\rho^{\prime}}$. Com isso provamos a

Proposiçāo 2.8 Se para alguma ação $\rho$ de $\mathfrak{g}$ em $V$ temos $H^{1}(\mathfrak{g}, V)=\{0\}$, então essa condição também vale para todas as ações $\rho^{\prime}$ suficientemente próximas de $\rho$. Mais ainda, $H^{0}(\mathfrak{g}, V)_{\rho^{\prime}}$ terá a mesma dimensão que $H^{0}(\mathfrak{g}, V)_{\rho}$.

Teorema 2.9 Seja $p \in M$ um zero elementar de uma ação A. Então existe uma vizinhança $N$ de $p$ onde todos os zeros são elementares. Além disso, o conjunto de zeros em $N$ é uma subvariedade $Z$ tal que $T_{p} Z=H^{0}\left(\mathfrak{g}, T_{p} M\right)$. 
Prova. Desde que a situação é local, podemos assumir, usando cartas locais, que $M$ é um subconjunto aberto de $V$ e $T_{p} M$ é identificado com $V$. Desde que $p$ é elementar, por 2.4 , a sequência

$$
V \stackrel{\alpha}{\longrightarrow} \operatorname{Hom}(\mathfrak{g}, V) \stackrel{\beta}{\longrightarrow} W
$$

é exata. Considere $S=\alpha(V)$ e escolha um subespaço complementar de $S$ e denote por $\pi_{c}$ a correspondente projeção de $\operatorname{Hom}(\mathfrak{g}, V)$ sobre $S$. O índice $c$ é para lembrar que a projeção depende da escolha do espaço complementar.

Usando uma base ordenada $\left\{X_{1}, \ldots, X_{l}\right\}$ de $\mathfrak{g}$, identificamos cada $f \in H o m(\mathfrak{g}, V)$ $\operatorname{com}\left(f\left(X_{1}\right), \ldots, f\left(X_{l}\right)\right) \in V^{l}$. Sendo $\alpha(u)$ uma aplicação linear de $\mathfrak{g}$ em $V$, temos que $\alpha(u)=\left(\alpha(u)\left(X_{1}\right), \ldots, \alpha(u)\left(X_{l}\right)\right)=\left(D A\left(X_{1}\right)_{p}(u), \ldots, D A\left(X_{l}\right)_{p}(u)\right)$. Portanto

$$
\alpha=\left(D A\left(X_{1}\right)_{p}, \ldots, D A\left(X_{l}\right)_{p}\right) .
$$

Defina $\varphi: M \rightarrow S: x \mapsto \pi_{c}\left(A\left(X_{1}\right)(x), \ldots, A\left(X_{l}\right)(x)\right)$. Logo, desde que $p$ é um zero de $A$, então $\varphi(p)=0$. Como $D \varphi_{p}=\pi_{c}\left(D A\left(X_{1}\right)_{p}, \ldots, D A\left(X_{l}\right)_{p}\right)=\pi_{c} \circ \alpha$ temos que $D \varphi_{p}$ é sobrejetiva. Pelo Teorema das Funções Implícitas, existe uma vizinhança $N$ de $p$ tal $Z=\{x \in N: \varphi(x)=0\}$ é uma subvariedade tangente a $\mathfrak{K e r} D \varphi_{p}$ em $p$. Desde que $\pi_{c}$ é a projeção de $\operatorname{Hom}(\mathfrak{g}, V)$ sobre $S$, com relação a um subespaço complementar qualquer de $S$,

$$
\varphi(x)=0 \Leftrightarrow\left(A\left(X_{1}\right)(x), \ldots, A\left(X_{l}\right)(x)\right)=0 \Leftrightarrow x \text { é zero de } A \text {. }
$$

Portanto $Z$ é o conjunto de zeros em $N$. Além disso, pelo mesmo argumento,

$$
\mathfrak{K e r} D \varphi_{p}=\mathfrak{K e r} \alpha=H^{0}(\mathfrak{g}, V)
$$

ou seja, $T_{p} Z=H^{0}(\mathfrak{g}, V)$.

Finalmente, do fato que $A$ é de classe $C^{1}$, para todo ponto $p^{\prime}$ próximo de $p$, implica que $D A(X)_{p^{\prime}}$ está próximo de $D A(X)_{p}$. Logo, a Proposição 2.8 garante que, restringindo $N$ se necessário, todo zero de $N$ é elementar.

Corolário. Seja $A: \mathfrak{g} \rightarrow \Im^{1}(M)$ uma ação. Então:

(a) $\operatorname{Se}[\mathfrak{g}, \mathfrak{g}] \neq \mathfrak{g}$, então os zeros elementares de $A$ são isolados.

(b) Se g é semi-simples então o conjunto de zeros de $A$ é uma subvariedade de $M$.

Prova. (a) A Proposição $2.4 \mathrm{diz}$ que $H^{0}\left(\mathfrak{g}, T_{p} M\right)=\{0\}$, e (a) segue do Teorema 2.9.

(b) Segue diretamente do Lema de Whitehead (que garante que $H^{1}(\mathfrak{g}, V)=$ $\{0\}$ se g é semi-simples) e do Teorema 2.9. 
A parte (a) do corolário diz que não precisavamos ter calculado $H^{1}$ para as ações dos exemplos C, D e E, páginas 17-20, uma vez que os zeros dessas ações não são isolados. Um exemplo de uma ação com uma subvariedade unidimensional de zeros elementares é a ação de $\mathfrak{s}^{3}$ do Exemplo F.

Corolário 2.10 Seja $A: \mathfrak{g} \rightarrow \Im^{1}(M)$ uma ação e $\mathfrak{g}$ uma álgebra de Lie tal que $[\mathfrak{g}, \mathfrak{g}]=\mathfrak{g}$. Se $p \in M$ é um zero para $A$ tal que a parte linear é trivial, então a ação $A$ é trivial sobre a componente conexa de $M$ que contém $p$.

Prova. Se $p \in M$ é um zero tal que a parte linear é trivial, então

$$
\rho(X) u=0, \forall X \in \mathfrak{g}, u \in T_{p} M,
$$

onde $\rho$ é a parte linear de $A$ em $p$. Logo, da definição, $H^{0}\left(\mathfrak{g}, T_{p} M\right)=T_{p} M$. Além disso, pelo Exemplo Açāo Trivial, $H^{1}\left(\mathfrak{g}, T_{p} M\right)=\operatorname{Hom}\left(\frac{\mathfrak{g}}{[\mathfrak{g}, \mathfrak{g}]}, T_{p} M\right)$. Desde que $[\mathfrak{g}, \mathfrak{g}]=\mathfrak{g}$, então $H^{1}\left(\mathfrak{g}, T_{p} M\right)=\{0\}$ e portanto $p$ é um zero elementar. Como $H^{0}\left(\mathfrak{g}, T_{p} M\right)=T_{p} M$, o Teorema 2.9 implica que o conjunto de zeros de $A$ contém uma vizinhança de $p$.

Portanto, o conjunto de pontos em $M$ tal que $A$ e sua parte linear são triviais é um aberto em $M$ que contém $p$. Desde que esse conjunto também é fechado, ele deve conter a componente conexa que contém $p$.

Tomemos a topologia do espaço das ações tal que se $\left\{X_{1}, \ldots, X_{l}\right\}$ é uma base ordenada para $\mathfrak{g}$ então uma sequência de ações $\left\{A_{i}\right\}$ convergem se $A_{i}\left(X_{j}\right)$ e $D_{k} A_{i}\left(X_{j}\right), j=1, \ldots, l, k=1,2$ convergem uniformemente sobre conjuntos compactos.

Teorema 2.11 Seja p uma zero elementar de uma ação $A: \mathfrak{g} \rightarrow \Im^{2}(M)$. Então, dado uma vizinhança $N$ de $p$, existe uma vizinhança $U$ de $A$ no espaço das açoes $C^{2}$ tal que para todo $A^{\prime} \in U$ existe $p^{\prime} \in N$ que é um zero elementar de $A^{\prime}$.

Prova. Desde que o problema é local, e analogamente à demontração do Teorema 2.9, considere $\rho$ a parte linear de $A$ em $p, S=\alpha(V)(=\mathfrak{K e r} \beta), \pi$ : $\operatorname{Hom}(\mathfrak{g}, V) \rightarrow S$ a projeção com relação a um subespaço complementar de $S$, $\varphi: M \rightarrow S: x \mapsto \pi\left(A\left(X_{1}\right)(x), \ldots, A\left(X_{l}\right)(x)\right)$ onde $\left\{X_{1}, \ldots, X_{l}\right\}$ é base de $\mathfrak{g}$.

Definimos a aplicação $\varphi^{\prime}: M \rightarrow S: x \mapsto \pi\left(A^{\prime}\left(X_{1}\right)(x), \ldots, A^{\prime}\left(X_{l}\right)(x)\right)$ para uma ação $A^{\prime}$ próxima de $A$. Logo, dada uma vizinhança $N$ de $p$ tomemos $A^{\prime}$ suficientemente próxima de $A$ tal que $\varphi^{\prime}$ está uniformemente próxima de $\varphi$ em $N$. Desde que $D \varphi(p)=\pi \circ \alpha$ é sobrejetora, $\varphi(N)$ contém uma vizinhança de 0 . Então podemos supor que existe $p^{\prime} \in N$ tal que $\varphi^{\prime}(p)=0$, isto é, $p^{\prime}$ é um zero de $A^{\prime}$. Como em 2.9 considere $\rho^{\prime}$ a parte linear de $A^{\prime}$ em $p^{\prime}$. A topologia no espaço das ações e a desigualdade do Valor Médio garantem que $\rho^{\prime}$ é uma ação de $\mathfrak{g}$ em $V$ próxima de $\rho$. Logo, da Proposição 2.8 segue que $p^{\prime}$ é zero elementar de $A^{\prime}$. 
Corolário. Seja $A: \mathfrak{g} \rightarrow \Im^{2}(M)$ uma ação tal que $[\mathfrak{g}, \mathfrak{g}]=\mathfrak{g}$. Se $p \in M$ é um zero para $A$ tal que a parte linear é trivial, então existe uma vizinhança $U$ de $A$ tal que $A^{\prime}(X) p^{\prime}=0$ se $X \in \mathfrak{g}, A^{\prime} \in U$, e $p^{\prime}$ está na mesma componente conexa de $p$.

Prova. Como em 2.10 as hipóteses implicam que $H^{0}\left(\mathfrak{g}, T_{p} M\right)=T_{p} M$ e $H^{1}\left(\mathfrak{g}, T_{p} M\right)=0$, isto é, $p$ é zero elementar de $A$. Pelo Teorema 2.11 existe uma vizinhança $U$ de $A$, no espaço das ações $C^{2}$, tal que para todo $A^{\prime} \in U$ existe um $p^{\prime}$ numa vizinhança de $p$ que é um zero elementar para $A^{\prime}$. A Proposição 2.8 garante que $H^{0}\left(\mathfrak{g}, T_{p^{\prime}} M\right)_{\rho^{\prime}}=T_{p^{\prime}} M$ para $\rho^{\prime}$ parte linear de $A^{\prime}$ em $p^{\prime}$. Logo, o Teorema 2.9 diz que o conjunto de zeros de $A^{\prime}$ inclui um aberto de $M$. Desde que este conjunto também é fechado, ele deve conter uma componente conexa, a mesma de $p$.

\section{O Teorema Principal}

Os próximos resultados serão utilizados na demonstração de 2.15. Seja $U$ um aberto de $\mathbb{R}$. Denotemos por $\mathcal{C}^{r}(U, \mathbb{R})$ o conjuto das aplicações de classe $C^{r}$ de $U$ em $\mathbb{R}$. O próximo resultado é um caso particular do Teorema da Densidade Transversal.

Teorema 2.12 Sejam $\bar{G}: \mathbb{R}^{2} \rightarrow \mathcal{C}^{1}(U, \mathbb{R}):(a, b) \mapsto G_{a b}$, e $G$ a aplicação avaliação de $\bar{G}$, isto é, $G: \mathbb{R}^{2} \times U \rightarrow \mathbb{R}$ tal que $G(a, b ; x)=G_{a b}(x)$. Suponhamos que $G$ é de classe $C^{1}$ e que 0 é valor regular de $G$. Então o conjunto

$$
\mathcal{A}=\left\{(a, b) \in \mathbb{R}^{2}: 0 \text { é valor regular para } G_{a b}\right\}
$$

tem a propriedade que $\mathbb{R}^{2}-\mathcal{A}$ tem medida nula. "

Prova. Ver [2, pg.48].

Lema 2.13 Seja $N$ uma variedade diferenciável $e\left\{U_{\lambda}: \lambda \in \Lambda\right\}$ um recobrimento aberto de $N$. Então, existe um atlas $\mathfrak{U}=\left\{h_{i}: V_{i} \rightarrow V_{i}^{\prime} \mid i \in \mathbb{N}\right\}$ de $N$ com as seguintes propriedades:

(a) $\left\{V_{i} \mid i \in \mathbb{N}\right\}$ é um refinamento localmente fino de $\left\{U_{\lambda} \mid \lambda \in \Lambda\right\}$;

(b) $V_{i}^{\prime}=\left\{\left(x_{1}, \ldots, x_{m}\right) \in \mathbb{R}^{m}: \max \left\{x_{1}, \ldots, x_{m}\right\}<3\right\}=: B(3)$;

(c) Os conjuntos $W_{i}:=h_{i}^{-1}(B(1))$ ainda cobrem $N$.

Prova. Ver [4].

Lema 2.14 Se $A: \mathfrak{g} \rightarrow \Im^{2}(M)$ como em 2.15 e dim $\mathfrak{g} \geq 2$ então para quase todo 2-plano $\mathcal{P} \subset \mathfrak{g}$ a restrição de $A$ a $\mathcal{P}$ tem precisamente as mesmas órbitas que $A$. 
Prova. Ver [15].

Teorema 2.15 Seja $A: \mathfrak{g} \rightarrow \Im^{2}(M)$ uma ação onde $\mathfrak{g}$ é abeliano e $M$ é uma superfície compacta. Assuma que todo zero de A é elementar. Então existe $\mathcal{S} \subset \mathfrak{g}$ tal que $\mathfrak{g}-\mathcal{S}$ tem medida zero e para $X \in \mathcal{S}$ :

(a) O conjunto de zeros isolados de $X$ coincide com o conjunto (finito) de zeros de $A$

(b) Se $p_{1}, \ldots, p_{k}$ são os zeros de $A$ então $\sum_{i=1}^{k}$ indice $X\left(p_{i}\right)=\chi(M)$.

Prova. Em vista do Lema 2.14 podemos supor que $\operatorname{dim} \mathfrak{g}=2$ e portanto assumir que a ação é expandida por dois campos de vetores $Y$ e $Z$ que comutam. Suponhamos que todas as órbitas da ação tem dimensão 2 , isto é, $Y$ e $Z$ são l.i. em todo ponto. Então as órbitas de $M$ são toros e $M$ é união, finita, de toros. Portanto $\chi(M)=0$ e (a) e (b) valem pois a ação não tem zeros.

Denotemos por $\mathcal{O}_{0}$ o conjunto das órbitas de dimensão 0 e $\mathcal{O}_{1}$ o conjunto das órbitas de dimnsão 1 . Suponhamos $\mathcal{O}_{1}$, é não vazio e seja $p \in \mathcal{O}_{1}$. Suponhamos que $Y(p) \neq 0$. Pelo Teorema do Fluxo Tubolar existe um sistema de coordenadas locais $(x, y)$ para uma vizinahnça $U_{p}$ de $p$ tal que $Y=\frac{\partial}{\partial y}$. Em $U_{p}$ temos que $Z=f \frac{\partial}{\partial x}+g Y$ onde $f$ e $g$ são funções de classe $C^{1}$ em $U_{p}$. Como $Y$ e $Z$ comutam, então

$$
0=[Y, Z]=f_{y} \frac{\partial}{\partial x}+g_{y} Y .
$$

Logo, $f$ e $g$ dependem somente de $x$. Considere $a Y+b Z$ um campo de vetores arbitrário em $\mathfrak{g}$. Logo, a restrição de $a Y+b Z$ em $U_{p}$ tem a forma

$$
a Y+b Z=b f \frac{\partial}{\partial x}+(a+b g) Y .
$$

Fixe $a, b \in \mathbb{R}$ e defina $G_{a b}: U \rightarrow \mathbb{R}: x \rightarrow a+b g(x)$. Considere $G: \mathbb{R}^{2} \times$ $U \rightarrow \mathbb{R}$ tal que $G(a, b, x)=a+b g(x)$. Desde que 0 é valor regular para $G$, então para quase todo $(a, b) \in \mathbb{R}^{2}$ a aplicação $G_{a b}$ tem 0 como valor regular por 2.12. Para tais $(a, b)$ o conjunto de zeros do campo de vetores $a Y+b Z$ é uma subvariedade de dimensão 1 do conjunto $\left\{(x, y) \in U: G_{a b}(x)=0\right\}$. Desde que $Y$ nunca é zero em $U$ e $f$ não depende de $x$, então o conjunto de zeros de $a Y+b Z$ é uma subvariedade de dimensão 1 . Observe que não é necessariamente todo o conjunto $\left\{(x, y) \in U: G_{a b}(x)=0\right\}$.

Consideremos $N=M-\mathcal{O}_{0}, \mathcal{O}_{S}=\mathcal{O}_{0} \cup \mathcal{O}_{1}, V=M-\mathcal{O}_{S}$ e $\mathfrak{U}$ o recobrimento de $N$ constituido do aberto $V$ e dos abertos $U_{p}, \forall p \in \mathcal{O}_{1}$. Então existe, pelo Lema 2.13, um refinamento localmente finito e enumerável de $\mathfrak{U}$. Em particular existe uma coleção enumerável de abertos $\left\{U_{i}\right\}_{i \in \mathbb{N}}$ que cobrem $\mathcal{O}_{1}$. Desde que intersecção enumerável de conjuntos residuais é também residual, implica que existe um subconjunto $\mathcal{S}_{1} \subset \mathfrak{g}$ tal que $\mathfrak{g}-\mathcal{S}$ tem medida nula e $X \in \mathcal{S}_{1}$ implica que 
o conjunto de zeros de $X$ consiste do conjunto $\left\{p_{1}, \ldots, p_{k}\right\}$ junto com subvariedades de dimensão 1 de $M-\left\{p_{1}, \ldots, p_{k}\right\}$. Pela Proposição 2.7 existe um conjunto $\mathcal{S}_{0} \subset \mathfrak{g}$ tal que $\mathfrak{g}-\mathcal{S}_{0}$ tem medida nula e $X \in \mathcal{S}_{0}$ implica que $X$ tem $\left\{p_{1}, \ldots, p_{k}\right\}$ como conjunto de zeros elementares. Em particular $p_{1}, \ldots, p_{k}$ são zeros isolados para qualquer $X \in \mathcal{S}_{0}$. Para $X \in \mathcal{S}=\mathcal{S}_{0} \cap \mathcal{S}_{1}$ o conjunto de zeros consiste de $p_{1}, \ldots, p_{k}$ junto com uma subvariedade compacta de dimensão $1 \mathrm{em} M$ que deve ser uma união finita de órbitas circulares, o que concui (a).

Tais órbitas circulares $X$ tem vizinhanças tubolares duas a duas disjuntas, apresentando-se como um cilindro ou uma faixa de Möebius se a superfície for não orientável. Além disso, $X$ é transversal ao bordo de cada vizinhança. Seja $T$ a união, finita, de tais vizinhanças tubolares para $X \in \mathcal{S}$. Então $M-T$ é uma superfície com bordo tal que $\chi(M-T)=\chi(M)$ [10]. E mais, a restrição de $X$ à $M-T$ é transversal ao bordo e tem $\left\{p_{1}, \ldots, p_{k}\right\}$ como conjunto de zeros. A parte (b) segue da fórmula de Poincaré-Hopf para a superfície $M-T$.

A demonstração do Teorema mostra que o campo de vetores $X$ pode também ter zeros que não são isolados. Por exemplo, considere os campos de vetores sobre o plano

$$
X=(\cos 2 \pi x) \frac{\partial}{\partial y}, \quad Y=(\operatorname{sen} 2 \pi x) \frac{\partial}{\partial y} .
$$

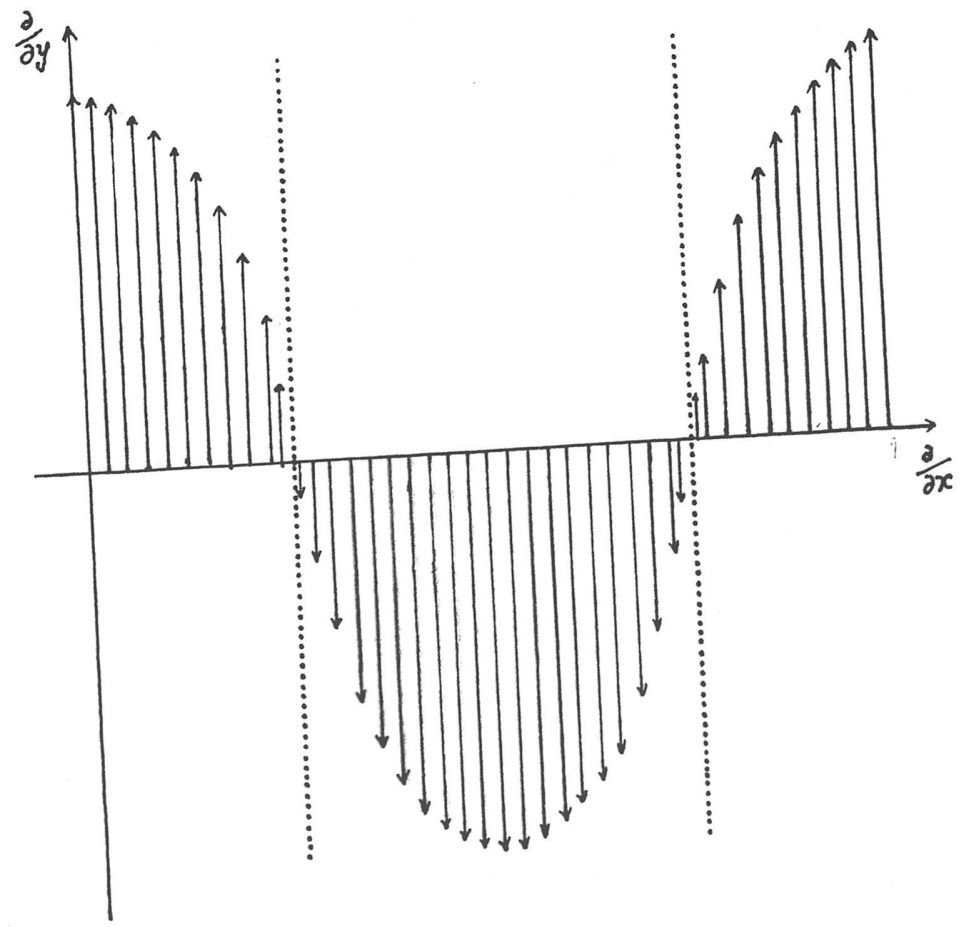

Campo $X$ 
Esses dois campos de vetores comutam e induzem campos de vetores $\widetilde{X}$ e $\tilde{Y}$ que comutam sobre o toro $T^{2}=\mathbb{R}^{2} / \mathbb{Z}^{2}$. Utilizando coordenadas polares, todo campo de vetores na álgebra de Lie $\mathfrak{g} \subset \Im^{1}(M)$ expandida por $\widetilde{X}$ e $\widetilde{Y}$ é um múltiplo escalar de algum $\widetilde{X}_{t}=(\cos 2 \pi t) \widetilde{X}+(\operatorname{sen} 2 \pi t) \widetilde{Y}=\cos 2 \pi(x-t) \frac{\partial}{\partial y}$. Logo, o conjunto de zeros de $\widetilde{X}_{t}$ é a união de dois circulos $x=t+\frac{1}{4}$ e $x=t+\frac{3}{4}$.

O teorema nos permite imaginar que seja possível generalizar a noção de índice para um zero isolado $p$ de uma ação por uma álgebra de Lie abeliana como sendo o índice de um elemento genérico $X \in \mathcal{S}$ em $p$. No entanto, no Exemplo G, temos uma ação de uma álgebra de Lie abeliana sobre os campos de vetores do toro $T^{2}$, expandida pelos campos $\widetilde{X}$ e $\widetilde{Y}$, tal que indice $(\widetilde{X}, p)=-$ indice $(\widetilde{Y}, p)= \pm 1$ para cada zero $p$. 


\section{Capítulo 3}

\section{Apêndice}

\section{Um pouco de Cohomologia.}

Denotemos por $\operatorname{Hom}_{\Lambda}(A, B)$ o conjunto de todos os homomorfismos do $\Lambda$ módulo $A$ para o $\Lambda$-módulo $B$, onde $\Lambda$ é um anel comutativo com unidade. Claramente este conjunto tem estrutura de grupo abeliano com a operação soma definida a seguir: se $\eta, \psi \in \operatorname{Hom}_{\Lambda}(A, B)$ então $(\eta+\psi)$ definido por $(\eta+\psi) a=$ $\eta a+\psi b, \forall a \in A$, também está em $\operatorname{Hom}_{\Lambda}(A, B)$. Omitiremos o simbolo $\Lambda$ a menos que seja necessário.

Seja $\beta: B_{1} \rightarrow B_{2}$ um homomorfismo de módulos. Então $\beta$ induz um homomorfismo de grupos abelianos $\beta_{*}$ definido por

$$
\begin{aligned}
\beta_{*}: \operatorname{Hom}\left(A, B_{1}\right) & \rightarrow \operatorname{Hom}\left(A, B_{2}\right) . \\
\varphi & \mapsto \beta \varphi
\end{aligned}
$$

Logo, definimos o functor (covariante) $\operatorname{Hom}(A,-)$ da categoria dos módulos na categoria dos grupos abelianos, que associa cada módulo $B$ com o grupo abeliano $\operatorname{Hom}(A, B)$, e cada homomorfismo de módulos $\beta$ o homomorfismo de grupos $\beta_{*}$.

Por outro lado, se $\alpha: A_{1} \rightarrow A_{2}$ é um homomorfismo de módulos, então $\alpha$ induz o seguinte homomorfismo de grupos:

$$
\begin{aligned}
\alpha^{*}: \operatorname{Hom}\left(A_{2}, B\right) & \rightarrow \operatorname{Hom}\left(A_{1}, B\right) . \\
\varphi & \mapsto \varphi \alpha
\end{aligned}
$$

Analogamente ao caso anterior, definimos o functor (contravariante) $\operatorname{Hom}(-, B)$ da categoria dos módulos na categoria dos grupos abelianos.

Sejam $\mu: A \rightarrow B$ e $\varepsilon: B \rightarrow C$ homomorfismos de módulos. A sequência $A \stackrel{\mu}{\rightarrow} B \stackrel{\varepsilon}{\rightarrow} C$ é chamada EXATA $($ em $B)$ se $\mathfrak{K e r} \varepsilon=\operatorname{Im} \mu$. Se a sequência $A_{0} \rightarrow$ $A_{1} \rightarrow \cdots \rightarrow A_{n} \rightarrow A_{n+1}$ é exata em $A_{1}, \ldots, A_{n}$, então a sequência é chamada simplesmente de exata. Por exemplo a sequência $0 \rightarrow A^{\prime} \stackrel{\mu}{\rightarrow} A \stackrel{\varepsilon}{\rightarrow} A^{\prime \prime} \rightarrow 0$ é exata (em $A^{\prime}, A, A^{\prime \prime}$ ) se e só se $\mu$ induz um isomorfismo $A^{\prime} \widetilde{\rightrightarrows} \mu A^{\prime}$ e $\varepsilon$ induz um isomorfismo $A / \mathfrak{K e r} \varepsilon=A / \mu A^{\prime} \widetilde{\rightrightarrows} A^{\prime \prime}$. Essencialmente $A^{\prime}$ é um submódulo de $A$, e $A^{\prime \prime}$ o correspondente módulo quociente. Tal sequência exata é chamada EXATA Curta e será denotada por $A^{\prime} \stackrel{\mu}{\longmapsto} A \stackrel{\varepsilon}{\rightarrow} A^{\prime \prime}$.

Teorema 3.1 Seja $B$ ’ $\stackrel{\mu}{\hookrightarrow} B \stackrel{\varepsilon}{\rightarrow} B$ ” uma sequência de módulos. Então para todo módulo $A$ a sequência

$$
0 \rightarrow \operatorname{Hom}\left(A, B^{\prime}\right) \stackrel{\mu_{*}}{\rightarrow} \operatorname{Hom}(A, B) \stackrel{\varepsilon_{*}}{\rightarrow} \operatorname{Hom}\left(A, B^{\prime \prime}\right)
$$


é exata.

Teorema 3.2 Seja $A^{\prime} \stackrel{\mu}{\longmapsto} A \stackrel{\varepsilon}{\rightarrow} A^{\prime \prime}$ uma sequência de módulos. Então para todo módulo $B$ a sequência

$$
0 \rightarrow \operatorname{Hom}\left(A^{\prime \prime}, B\right) \stackrel{\varepsilon^{*}}{\rightarrow} \operatorname{Hom}(A, B) \stackrel{\mu^{*}}{\rightarrow} \operatorname{Hom}\left(A^{\prime}, B\right)
$$

é exata.

As demonstrações podem ser vistas em [6].

Um módulo $P$ é chamado de Projetivo se para todo homomorfismo sobrejetivo de módulos $\varepsilon: B \rightarrow C$ e todo homomorfismo $\gamma: P \rightarrow C$ existe um homomorfismo $\pi: P \rightarrow B$ tal que $\varepsilon \pi=\gamma$. Exemplos de módulos projetivos são os $K$-módulos onde $K$ é um corpo.

Uma sequência exata curta $R \stackrel{\mu}{\longmapsto} P \stackrel{\varepsilon}{\leftrightarrow} A$ de $\Lambda$-módulos com $P$ projetivo é chamada uma Presentação Projetiva de $A$. Logo, quando $\Lambda=K$ qualquer sequência exata curta $A \longmapsto B \rightarrow C$ é uma presentação projetiva de $C$.

Pelo Teorema 3.2 uma presentação projetiva $R \stackrel{\mu}{\longmapsto} P \stackrel{\varepsilon}{\rightarrow} A$ induz, para um módulo $B$, uma sequência exata

$$
0 \rightarrow \operatorname{Hom}(A, B) \stackrel{\varepsilon^{*}}{\rightarrow} \operatorname{Hom}(P, B) \stackrel{\mu^{*}}{\rightarrow} \operatorname{Hom}(R, B) .
$$

Para os módulos $A$ e $B$, e para uma presentação projetiva escolhida de $A$ nós podemos associar o grupo abeliano

$$
\operatorname{Ext}(A, B)=\operatorname{Ext}_{\Lambda}(A, B)=\operatorname{Coker}\left(\mu^{*}: \operatorname{Hom}(P, B) \rightarrow \operatorname{Hom}(R, B)\right) .
$$

Prova-se que esta definição não depende da presentação projetiva escolhida. Um elemento em $\operatorname{Ext}(A, B)$ pode ser representado por $\varphi: R \rightarrow B$ e será denotado por $[\varphi] \in \operatorname{Ext}(A, B)$. Então $\left[\varphi_{1}\right]=\left[\varphi_{2}\right]$ se e somente se $\varphi_{1}-\varphi_{2}$ se extende a $P$.

Claramente um homomorfismo $\beta: B \rightarrow B^{\prime}$ aplica a sequência $3.1 \mathrm{em}$ uma sequência correspondente para $B^{\prime}$

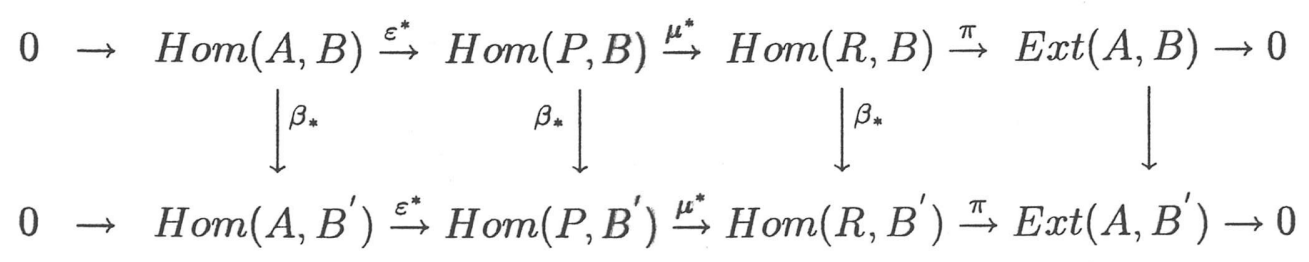

Assim, temos a aplicação induzida $\beta_{*}: \operatorname{Ext}(A, B) \rightarrow \operatorname{Ext}\left(A, B^{\prime}\right): \beta_{*}([\varphi]) \rightarrow[\beta \varphi]$ que é um homomorfismo de grupos abelianos e portanto é fácil ver que $\operatorname{Ext}(A,-)$ é um functor da categoria dos módulos na categoria dos grupos abelianos.

Por outro lado, se $R \stackrel{\mu}{\mapsto} P \stackrel{\varepsilon}{\rightarrow} A$ e $R^{\prime} \stackrel{\mu^{\prime}}{\mapsto} P^{\prime} \stackrel{\varepsilon^{\prime}}{\rightarrow} A^{\prime}$ são presentações projetivas de $A$ e $A^{\prime}$ e $\alpha: A \rightarrow A^{\prime}$ é um homomorfismo de módulos, desde que $P$ é projetivo 
existe um homomorfismo $\pi: P \rightarrow P^{\prime}$ induzindo $\sigma: R \rightarrow R^{\prime}$ tal que o seguinte diagrama é comutativo

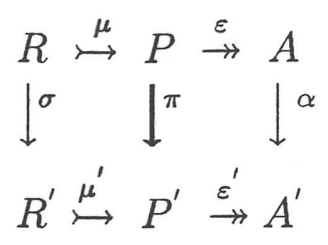

Analogamente ao caso anterior, para um módulo $B$, as aplicações $\alpha$ e $\pi$, junto com $\sigma$, induzem o seguinte diagrama de homomorfismos e grupos abelianos

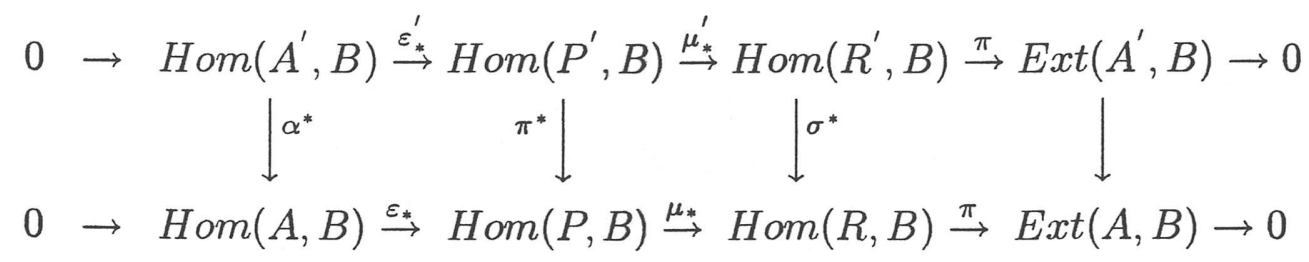

Portanto, existe um homomorfismo de grupos abelianos induzido denotado por $\alpha^{*}: \operatorname{Ext}(A, B) \rightarrow \operatorname{Ext}\left(A^{\prime}, B\right)$. Prova-se que este homomorfismo só depende de $\alpha$, o que justifica a notação. Portanto $\operatorname{Ext}(-, B)$ é um functor (contravariante) da categoria dos módulos na categoria dos grupos abelianos.

Teorema 3.3 Sejam $A$ um módulo e $B^{\prime} \stackrel{\mu}{\longmapsto} B^{\varepsilon} \stackrel{\varepsilon}{\rightarrow} B^{\prime \prime}$ uma seqüência exata curta de módulos. Então existe um homomorfismo $\omega: \operatorname{Hom}\left(A, B^{\prime \prime}\right) \rightarrow \operatorname{Ext}\left(A, B^{\prime}\right)$ tal que a seguinte sequência é exata

$$
\begin{aligned}
0 \longrightarrow & \operatorname{Hom}\left(A, B^{\prime}\right) \stackrel{\mu_{*}}{\longrightarrow} \operatorname{Hom}(A, B) \stackrel{\varepsilon_{*}}{\longrightarrow} \operatorname{Hom}\left(A, B^{\prime \prime}\right) \\
& \stackrel{\omega}{\longrightarrow} \operatorname{Ext}\left(A, B^{\prime}\right) \stackrel{\mu_{*}}{\longrightarrow} \operatorname{Ext}(A, B) \stackrel{\varepsilon_{*}}{\longrightarrow} \operatorname{Ext}\left(A, B^{\prime \prime}\right) .
\end{aligned}
$$

Essa sequência é chamada SEQüÊNCIA HoM-EXT (na segunda variável).

Dado $\alpha: A \rightarrow B$ " nós definimos as aplicações $\pi, \sigma$ tal que o seguinte diagrama

$$
\begin{gathered}
R \stackrel{\mu}{\longmapsto} P \stackrel{\varepsilon}{\rightarrow} A \\
\left.\left.\left.\right|^{\sigma} \quad\right|^{\pi}\right|^{\alpha} \\
B^{\prime} \stackrel{\mu^{\prime}}{\mapsto} B \stackrel{\varepsilon^{\prime}}{\rightarrow} B^{\prime \prime}
\end{gathered}
$$

é comutativo. A demonstração do teorema (ver [6]) mostra que $\omega(\alpha)=[\sigma] \in$ $\operatorname{Ext}\left(A, B^{\prime}\right)$.

Uma categoria Aditiva $\mathfrak{U}$ é uma categoria com objeto zero tal que quaisquer dois objetos tem um produto e no qual o conjunto de morfismos $\mathfrak{U}(A, B)$ são grupos abelianos tal que a composição

$$
\mathfrak{U}(A, B) \times \mathfrak{U}(B, C) \rightarrow \mathfrak{U}(A, C)
$$


é bilinear. Um $\Lambda$-módulo Graduado é uma família de $\Lambda$-módulos $A=\left\{A_{n}\right\}_{n \in \mathbb{Z}}$. Se $A$ e $B$ são duas famílias de $\Lambda$-módulos graduados, um morfismo $\varphi: A \rightarrow B$ DE GRAU $k$ é uma família de homomorfismo de $\Lambda$-módulos $\left\{\varphi_{n}: A_{n} \rightarrow B_{n+k}\right\}_{n \in \mathbb{Z}}$. Essa categoria será denotada por $\mathfrak{M}_{\Lambda}^{\mathbb{Z}}$ e é uma categoria aditiva se nos restringirmos aos morfismos de grau 0 . O próximo resultado pode ser encontrado em [6]:

"Seja $\mathbf{F}: \mathfrak{U} \rightarrow \mathfrak{B}$ um functor de uma categoria aditiva $\mathfrak{U}$ em uma categoria aditiva $\mathfrak{B}$. Então as seguintes condições são equivalentes:

- F preserva somas (de dois objetos);

- F preserva produtos (de dois objetos);

- para cada $A, A^{\prime}$ em $\mathfrak{U}, \mathbf{F}: \mathfrak{U}\left(A, A^{\prime}\right) \rightarrow \mathfrak{B}\left(\mathbf{F} A, \mathbf{F} A^{\prime}\right)$ é um homomorfismo. "

Um functor satisfazendo qualquer das três condições acima é chamado de FUNCTOR ADITIVO.

Um Complexo de CADEIA $\mathbf{C}=\left\{C_{n}, \partial_{n}\right\}$ sobre $\Lambda$ é um objeto em $\mathfrak{M}_{\Lambda}^{\mathbb{Z}}$ junto com um endomorfismo $\partial: \mathbf{C} \rightarrow \mathbf{C}$ de grau -1 com $\partial \partial=0$, que chamamos de OPERADOR BORDO. Um morfismo $\varphi: \mathbf{C} \rightarrow \mathbf{D}$ de grau 0 em $\mathfrak{M}_{\Lambda}^{\mathbb{Z}}$ é dita uma APLICAÇÃo DE CADEIAS se $\varphi \partial=\widetilde{\partial} \varphi$ onde $\widetilde{\partial}$ é o operador bordo de D. Assim uma aplicação de cadeias $\varphi$ é uma famíla $\left\{\varphi_{n}: C_{n} \rightarrow D_{n}\right\}_{n \in \mathbb{Z}}$ de homomorfismos tal que, para todo $n$, o diagrama

$$
\begin{aligned}
& C_{n} \stackrel{\partial_{n}}{\rightarrow} C_{n-1} \\
& \left.\downarrow^{\varphi_{n}} \quad\right\rfloor^{\varphi_{n-1}} \\
& D_{n} \stackrel{\widetilde{\partial}_{n}}{\rightarrow} D_{n-1}
\end{aligned}
$$

é comutativo. Se $\mathbf{F}: \mathfrak{M}_{\Lambda} \rightarrow \mathfrak{M}_{\Lambda^{\prime}}$ é um functor covariante aditivo e se $\mathbf{C}=$ $\left\{C_{n}, \partial_{n}\right\}$ é um complexo de cadeia sobre $\Lambda$, então $\mathbf{F C}=\left\{\mathbf{F} C_{n}, \mathbf{F} \partial_{n}\right\}$ é um complexo de cadeia sobre $\Lambda^{\prime}$.

A condição que $\partial \partial=0$ implica que $\mathfrak{I m} \partial_{n+1} \subseteq \mathfrak{K e r} \partial_{n}, n \in \mathbb{Z}$. Portanto, nós podemos associar com $\mathbf{C}$ o módulo graduado

$$
\mathbf{H}(\mathbf{C})=\left\{H_{n}(\mathbf{C})\right\} \quad \text { onde } H_{n}(\mathbf{C})=\mathfrak{K e r} \partial_{n} / \mathfrak{I m} \partial_{n+1}, n \in \mathbb{Z} .
$$

Então $\mathbf{H}(\mathbf{C})\left(H_{n}(\mathbf{C})\right)$ é chamado de (n-ésimo) Módulo DE HoMologia de C. Os elementos de $\mathfrak{k e r} \partial_{n}$ são chamados de $n$-CiCLOS e os elementos de $\mathfrak{I m} \partial_{n}$ de $n$-BORDOS.

Se tomarmos $\mathbf{D}=\left\{D^{n}, \delta^{n}\right\}$ tal que $D^{n}=C_{-n}$ e $\delta^{n}=\partial_{-n}$ obtemos o que chamamos de Complexo DE CoCADEIA, ou seja $\mathbf{D}$ é um objeto em $\mathfrak{M}_{\Lambda}^{\mathbb{Z}}$ junto com um endomorfismo $\delta: \mathbf{D} \rightarrow \mathbf{D}$ de grau +1 tal que $\delta \delta=0$. Aqui $\delta$ é chamado de Operador Cobordo. AplicaÇÃo de Cocadeias é definido de modo 
análogo a aplicação de cadeias e definimos o MóDUlo DE COHOMOLOGIA de um complexo de cocadeia $\mathbf{D}$ por

$$
\mathbf{H}(\mathbf{D})=\left\{H^{n}(C)\right\} \quad \text { onde } H^{n}(C)=\mathfrak{K e r} \delta^{n} / \mathfrak{I m} \delta^{n-1}, n \in \mathbb{Z} \text {. }
$$

Os elementos de $\mathfrak{k e r} \delta^{n}$ são chamados de $n$-CociCLOS e os elementos de $\mathfrak{I m} \delta^{n}$ de $n$-CoBordos.

Por uma Resolução Projetiva de um $\Lambda$-módulo $A$, nós entendemos uma sequência exata

$$
\mathbf{P}: \cdots \rightarrow P_{n+1} \stackrel{\partial}{\rightarrow} P_{n} \stackrel{\partial}{\rightarrow} P_{n-1} \rightarrow \cdots
$$

tal que as seguintes condições se verificam:

- $P_{-1}=A$;

- $P_{n}=0$ para todo $n<-1$;

- $P_{n}$ é um $\Lambda$-módulo projetivo para todo $n \geq 0$.

Prova-se que todo $\Lambda$-módulo $A$ possui uma resolução projetiva (Ver [5]). Seja $B$ um $\Lambda$-módulo e $\mathbf{P}: 0 \leftarrow A \leftarrow P_{0} \leftarrow P_{1} \leftarrow \cdots$ uma resolução projetiva de $A$. Considere o seguinte complexo de cocadeia

$$
\operatorname{Hom}(\mathbf{P}, B): 0 \rightarrow \operatorname{Hom}_{\Lambda}\left(P_{0}, B\right) \rightarrow \operatorname{Hom}_{\Lambda}\left(P_{1}, B\right) \rightarrow \cdots
$$

Defina

$$
\operatorname{Ext}_{\Lambda}^{n}(A, B)=H^{n}\left(\operatorname{Hom}_{\Lambda}(P, B)\right), n=0,1, \ldots
$$

Da definição vemos que $\operatorname{Ext}_{\Lambda}^{n}(A, B)$ é um grupo abeliano e que $\operatorname{Ext}_{\Lambda}^{0}(A, B)=$ $\operatorname{Hom}_{\Lambda}(A, B)$ (isso decorre facilmente do teorema de isomorfismo). Não tão evidente é o fato que a definição não depende da escolha da resolução projetiva e que $\operatorname{Ext}_{\Lambda}^{1}(A, B)=\operatorname{Ext}_{\Lambda}(A, B)$, o que justifica a notação.

Seja $G$ um grupo. Relembrando que um $G$-módulo (a esquerda) é um grupo abeliano $A$ junto com um homomorfismo o: $G \rightarrow A u t(A)$, ou seja, o é uma ação de $G$ em $A$. A imagem de $a \in A$ por um automorfismo definido por $x$ é então denotado por $x \circ a$.

Seja $G$ um grupo e $A$ um $G$-módulo. Então definimos o $n$-ÉSIMO Grupo DE Cohomologia de $G$ com coeficientes em $A$ por

$$
H^{n}(G, A)=\operatorname{Ext}_{G}^{n}(\mathbb{Z}, A) .
$$

Logo, dado uma sequência exata curta $A^{\prime} \stackrel{\mu}{\longmapsto} A \stackrel{\varepsilon}{\rightarrow} A^{\prime \prime}$ a sequência Hom-Ext do Teorema 3.3 toma a forma

$$
\begin{aligned}
0 \rightarrow H^{0}\left(G, A^{\prime}\right) & \stackrel{\mu_{*}}{\rightarrow} H^{0}(G, A) \stackrel{\varepsilon_{*}}{\rightarrow} H^{0}\left(G, A^{\prime \prime}\right) \\
& \stackrel{\omega}{\rightarrow} H^{1}\left(G, A^{\prime}\right) \stackrel{\mu_{*}}{\rightarrow} H^{1}(G, A) \stackrel{\varepsilon_{*}}{\rightarrow} H^{1}\left(G, A^{\prime \prime}\right) .
\end{aligned}
$$


Desde que $\mathbb{Z}$ é um $G$-módulo trivial, isto é, o é trivial, o fato que todo homomorfismo de $G$-módulos $\varphi: \mathbb{Z} \rightarrow A$ é completamente determinado pela imagem de $1 \in \mathbb{Z}, \varphi(1)=a \in A$, implica que $x \circ a=x \circ \varphi(1)=\varphi(x \circ 1)=\varphi(1)=a$, onde a terceira igualdade se deve ao fato de $\mathbb{Z}$ ser um $G$-módulo trivial. Portanto

$$
H^{0}(G, A)=H_{G}(\mathbb{Z}, A)=\{a \in A: x \circ a=a, \forall x \in G\}
$$

ou seja $H^{0}(G, A)$ é o subgrupo dos elementos invariantes em $A$, pela ação o.

Até o final do apêndice, $K$ é um corpo, ge é uma álgebra de Lie sobre $K$ e $A$ é um $\mathfrak{g}$-módulo, isto é, $A$ é um $K$-espaço vetorial $A$ tal que existe um homomorfismo de álgebras de Lie $\rho: \mathfrak{g} \rightarrow \mathfrak{g l}(A)$. Definimos o $n$-ÉSIMO Grupo DE COHOMOLOGIA de $\mathfrak{g}$ com coeficientes em $A$ da mesma maneira que para um grupo, isto é,

$$
H^{n}(\mathfrak{g}, A)=\operatorname{Ext}_{\mathfrak{g}}^{n}(K, A), n=0,1, \ldots
$$

onde $K$ é naturalmente visto como um g-módulo trivial. Na verdade $H^{n}(\mathfrak{g}, A)$ é um $K$-espaço vetorial mas continuaremos chamando de grupo de cohomologia..

Analogamente ao caso anterior (a prova é a mesma), temos que

$$
H^{0}(\mathfrak{g}, A)=\{a \in A: \rho(x)(a)=a, \forall x \in G\}
$$

e dado uma sequência exata curta $A^{\prime} \stackrel{\mu}{\longmapsto} A \stackrel{\varepsilon}{\rightarrow} A^{\prime \prime}$ a sequência Hom-Ext do Teorema 3.3 toma a forma

$$
\begin{aligned}
0 \rightarrow H^{0}\left(\mathfrak{g}, A^{\prime}\right) & \stackrel{\mu_{*}}{\rightarrow} H^{0}(\mathfrak{g}, A) \stackrel{\varepsilon_{*}}{\rightarrow} H^{0}\left(\mathfrak{g}, A^{\prime \prime}\right) \\
& \stackrel{\omega}{\rightarrow} H^{1}\left(\mathfrak{g}, A^{\prime}\right) \stackrel{\mu_{*}}{\rightarrow} H^{1}(\mathfrak{g}, A) \stackrel{\varepsilon_{*}}{\rightarrow} H^{1}\left(\mathfrak{g}, A^{\prime \prime}\right) .
\end{aligned}
$$

Uma DERIVAÇÃo de uma álgebra de Lie g em um g-módulo $A$ é uma aplicação $K$-linear $f: \mathfrak{g} \rightarrow A$ tal que

$$
f([x, y])=\rho(x) f(y)-\rho(y) f(x) \quad x, y \in \mathfrak{g} .
$$

$\mathrm{O}$ conjunto das derivações tem uma estrutura de $K$-espaço vetorial e será denotado por $\operatorname{Der}(\mathfrak{g}, \mathrm{A})$. Desde que $A$ é um $\mathfrak{g}$-módulo se a aplicação $\rho(x)(a)$ é $K$-linear em $x$ e $a$ e

$$
\rho([x, y])(a)=\rho(x)(\rho(y)(a))-\rho(y)(\rho(x)(a)) \quad x, y \in \mathfrak{g}, a \in A,
$$

temos que para $a \in A$ fixo, a aplicação $f_{a}: \mathfrak{g} \rightarrow A$ definida por $f_{a}(x)=\rho(x)(a)$ é claramente uma derivação. Derivações deste tipo são chamadas de INTERNAS. O conjunto das derivações internas formam um subespaço em $\operatorname{Der}(\mathfrak{g}, A)$ denotado por $\operatorname{Ider}(\mathfrak{g}, \hat{A})$. Com um pouco de trabalho prova-se que

$$
H^{1}(\mathfrak{g}, A) \cong \operatorname{Der}(\mathfrak{g}, A) / \operatorname{Ider}(\mathfrak{g}, A)
$$


Considere $\mathfrak{g}$ uma álgebra de Lie, $\mathfrak{h}$ um ideal de $\mathfrak{g}$ e $\rho: \mathfrak{g} \rightarrow \mathfrak{g l}(V)$ um homomorfismo de Álgebras de Lie. Definimos uma aplicação chamada InFLAÇão como sendo

$$
\text { inf: } \begin{aligned}
H^{1}\left(\mathfrak{g} / \mathfrak{h}, H^{0}(\mathfrak{h}, V)\right) & \rightarrow H^{1}(\mathfrak{g}, V) \\
f & \longmapsto i \circ f \circ \pi
\end{aligned}
$$

onde $\pi$ é a projeção quociente e $i$ é a inclusão. Definimos também a aplicação RESTRIÇÃO

$$
\text { res: } \begin{aligned}
H^{1}(\mathfrak{g}, V) & \rightarrow H^{1}(\mathfrak{h}, V) \\
f & \left.\longmapsto f\right|_{\mathfrak{h}}
\end{aligned}
$$

Proposiçāo 3.4 A sequência

$$
0 \longrightarrow H^{1}\left(\mathfrak{g} / \mathfrak{h}, H^{0}(\mathfrak{h}, V)\right) \stackrel{\text { inf }}{\longrightarrow} H^{1}(\mathfrak{g}, V) \stackrel{\text { res }}{\longrightarrow} H^{1}(\mathfrak{h}, V)
$$

é exata.

Prova. A aplicação inflação é claramente injetiva. Desde que $\pi: \mathfrak{g} \rightarrow$ $\mathfrak{g} / \mathfrak{h}$ restrito à $\mathfrak{h}$ é a aplicação nula, $\left.i \circ f \circ \pi\right|_{\mathfrak{h}}=0$. Portanto $\mathfrak{I m}$ inf $\subset$ Ker res. Para provarmos a inclusão contrária, considere $g \in H^{1}(\mathfrak{g}, V)$ tal que $g \in \mathfrak{K e r}$ res. Precisamos encontrar um candidato $\bar{g} \in H^{1}\left(\mathfrak{g} / \mathfrak{h}, H^{0}(\mathfrak{h}, V)\right)$ tal que inf $(\bar{g})=g$. Para isso, tome

$$
\bar{g}: \begin{aligned}
& \mathfrak{g} / \mathfrak{h} \rightarrow H^{0}(\mathfrak{h}, V) \\
& \bar{Y} \longmapsto g(Y)
\end{aligned} .
$$

Como $i \circ \bar{g} \circ \pi(X)=i \circ \bar{g}(\bar{X})=i \circ g(X)=g(X)$, temos que $\bar{g}$ é um bom candidato. Mostremos que $\bar{g}$ está bem definida. Considere $Y, Y^{\prime} \in \mathfrak{g}$ tal $Y-Y^{\prime} \in \mathfrak{h}$. Então $\bar{g}(\bar{Y})-\bar{g}\left(\bar{Y}^{\prime}\right)=g(Y)-g\left(Y^{\prime}\right)=g\left(Y-Y^{\prime}\right)=0$, pois $g$ pertence à $\mathfrak{K e r}$ res. Falta mostrar apenas que $\bar{g}(\bar{Y}) \in H^{0}(\mathfrak{h}, V)$.

$$
\bar{g}(\bar{Y}) \in H^{0}(\mathfrak{h}, V) \Leftrightarrow \rho(X) \bar{g}(\bar{Y})=0, \forall X \in \mathfrak{h} \Leftrightarrow \rho(X) g(Y)=0, \forall X \in \mathfrak{h} .
$$

Desde que $[X, Y] \in \mathfrak{h}$ implica que $g([X, Y])=0$, pois $g \in \mathfrak{K e r}$ res, e $g([X, Y])=$ $\rho(X) g(Y)-\rho(Y) g(X)=\rho(X) g(Y)$ temos que $\rho(X) g(Y)=0, \forall X \in \mathfrak{h}$. Portanto, $\bar{g}$ está bem definida e $\mathfrak{k e r}$ res $\subset$ Im inf. 


\section{Bibliografia}

[1] L.Auslander, L.Green and F.Hahn, "Flows on Homogeneous Spaces", Annals of Math. Studies \#53, Princeton University Press, 1963.

[2] R.Abraham and J.Robbin, "Transversal Mappings and Flows", Benjamin, New York, 1967.

[3] F.Brickell and R.Clark, "Differential Manifolds, An Introduction", Van Nostrand-Reinhold Company, London, 1970.

[4] T.Bröcker and K.Jänich, "Introduction to Differential Topology", Cambridge University Press, 1982.

[5] S.-T.Hu, "Introduction to Homological Algebra", Holden-Day, San Francisco, 1968.

[6] P.J.Hilton and U.Stammbach, "A course in Homological Algebra", Springer, New York, 1971.

[7] H.Hopf, "Vektorfelder in n-dimensionaler Mannigfaltigkeiten", Math. Ann. 96, 1927, 225-250.

[8] N.Jacobson, "Lie Algebras", Interscience, New York, 1962.

[9] E.L.Lima, "Common Singularities of Commuting Vector Fields on 2Manifolds", Comm. Math. Helv. 39, 1964, 97-110. 
0] W.S.Massey, "Algebraic Topology: An Introduction", Harcourt, Brace \& World, Inc., New York, 1967.

[11] J.F.Plante, "Fixed Points of Lie Group Actions on Surfeces", Ergodic Th. \& Dynam. Systs., 6, 149-161, 1986.

[12] J.F.Plante, "Elementary Zeros of Lie Algebras of Vector Fields", Topology, 30(2), 215-222, 1991.

[13] R.Palais, "A Global Formulation of the Lie Theory of Transformation Groups", Mem. Amer. Math. Soc. \#22. Providence, 1957.

[14] H.Poincaré, "Sur les Curbes Définies par une Équation Differentielle", J. Math. Pures Appl. 4(1)1885, 167-244.

[15] R.Sacksteder, "Degeneracy of Orbits of Actions of $\mathbb{R}^{n}$ on a Manifold", Comm. Math. Helv., 41(1), 1-9, 1966.

[16] A.A.Sagle and R.E.Walde, "Introduction to Lie Groups and Lie Algebras", Academic Press, New York and London, 1973. 